\title{
Illustrated checklist of Anguilliformes (Pisces, Teleostei) of the lagoon of Mayotte (Western Indian Ocean) with 14 new records
}

\author{
RÉMY EUDELINE* \\ 117 Rue Jean Vallier, 69007 - Lyon, France. ORCID Rémy Eudeline (D) https://orcid.org/0000-0002-0375-9587
}

Marine and

Fishery Science MAFIS

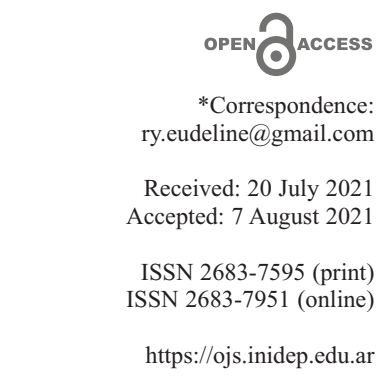

Journal of the Instituto Nacional de Investigación y Desarrollo Pesquero (INIDEP)

This work is licensed under a Creative Commons Attribution-

NonCommercial-ShareAlike 4.0 International License

\begin{abstract}
The Anguilliformes of Mayotte (French island of the Comoros Archipelago) remain poorly surveyed and poorly known so far. This paper deals with the diversity of the Order Actinopterygii of the lagoon of Mayotte by combining literature data, my own underwater visual censuses, and records from other divers. Most species were identified by in situ photographs. A total of 47 species were listed in detail with picture plates, including 14 species not previously mentioned for the lagoon of Mayotte. This paper shows that the use of citizen science, by providing pictures of observed species, has enormous potential to improve our understanding of the species community from an insufficiently surveyed region.
\end{abstract}

Key words: Undescribed species, updated distribution range, identification method, Moray eels, underestimated biodiversity.

Lista ilustrada de Anguiliformes (Peces, Teleósteos) de la laguna de Mayotte (Océano Índico Occidental) con 14 registros nuevos

RESUMEN. Los Anguilliformes de Mayotte (isla francesa del archipiélago de las Comoras) continúan siendo poco estudiados y apenas conocidos. En este trabajo, se investigó la diversidad del Orden Actinopterygii de la laguna de Mayotte combinando datos provenientes de la literatura, censos visuales submarinos propios y registros de otros buceadores. La mayoría de los individuos registrados se identificaron mediante fotografías in situ. Se enumeraron un total de 47 especies, detalladas con láminas ilustradas, incluidas 14 especies sin mención previa para la laguna de Mayotte. En este artículo se demuestra que el uso de la ciencia ciudadana, como en este caso las imágenes suministradas de las especies observadas, tiene un enorme potencial para mejorar nuestra comprensión de la comunidad de especies en una región insuficientemente estudiada.

Palabras clave: Especies no descriptas, distribución actualizada, método de identificación, morenas, biodiversidad subestimada.

\section{INTRODUCTION}

Mayotte $\left(12^{\circ} 50^{\prime} 35^{\prime \prime} \mathrm{S}-45^{\circ} 08^{\prime} 18^{\prime \prime} \mathrm{E}\right)$ is an overseas region and county of France. It is located in the Northern Mozambique Channel in the Western Indian Ocean off the coast of Southeast Africa, between Northwestern Madagascar and Northeastern Mozambique (Figure 1). It comprises a small archi- 


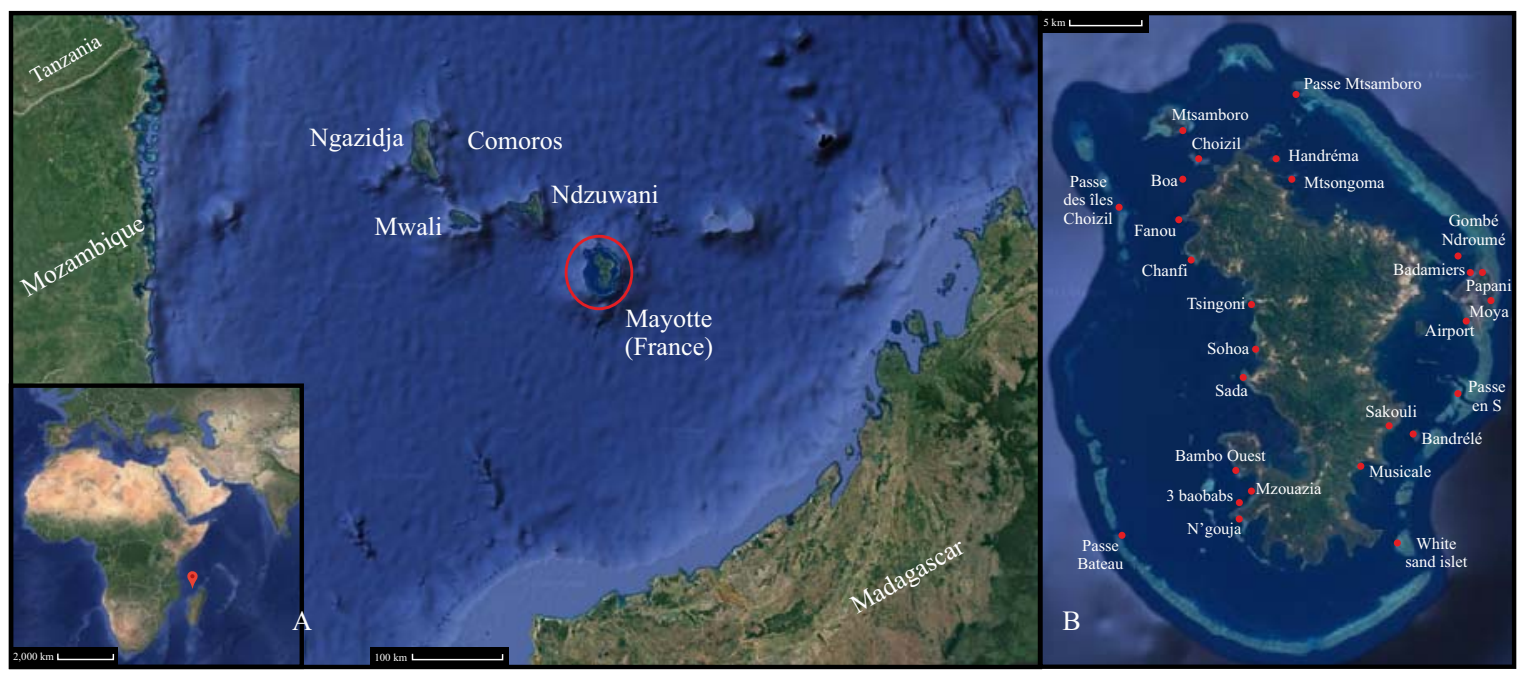

Figure 1. A) Location of Mayotte in the middle of the Mozambique Channel between the coasts of Madagascar to the East and Africa in the West. B) Map of Mayotte with indication of the study area (extracted and adapted from Google Earth http://earth.google.com/).

pelago of $376 \mathrm{~km}^{2}$ made up of two main islands, Grande-Terre and Petite-Terre, and several islets around them. A barrier reef of $195 \mathrm{~km}$ encloses the archipelago forming the largest lagoon of the Indian Ocean covering $1,100 \mathrm{~km}^{2}$. This volcanic island, with an age of around $20 \mathrm{Ma}$ (Michon 2016), is the oldest and easternmost of the four islands forming the Comoros Archipelago and is inhabited by 290,000 people (INSEE 2021).

Biodiversity of Mayotte lagoon, and the Comoros Archipelago in general, has attracted little attention from the scientific community compared to other oceanic volcanic islands. The result is a lack of knowledge regarding the biodiversity that these islands harbor. The marine ichthyofauna of the Comoros was studied by Fourmanoir (1954). Mayotte received dedicated attention only in the late 1970s (Wickel et al. 2014). Wickel (2004) produced the first synthesis of ichthyological data. In his checklist of fishes of Madagascar, Fricke et al. (2018) made a comparison of families of marine shore fishes around islands in the Southwestern Indian Ocean. Despite these previous works, Comoros and Mayotte lagoon are still relatively unexplored.
Some groups of low commercial interest, such as the Order Anguilliformes, remain particularly understudied in this area. One reason for this little knowledge stems from the fact that most general ichthyofaunistic studies focus on diurnal surveys to maximize the observation of fish activity during the day. However, many Anguilliformes are nocturnal and, therefore, often remain undetected. An updated checklist describing the diversity of the Order Anguilliformes from Mayotte lagoon is presented herein by combining data from the literature and underwater photographic and visual censuses.

\section{MATERIALS AND METHODS}

For each species, the first published record from Mayotte is provided based on literature (Böhlke and Randall 2000; Deliot 2000; Fouquet 2000; Keith et al. 2006; Wickel and Jamon 2010; Wickel et al. 2014; Allaria 2016). Several unpublished reports previously compiled in Wickel et al. (2014) were also used. 
Data collected from underwater visual censuses represented a minimum of $352 \mathrm{~h}$ of observation (total diving: $64 \mathrm{~h}$ during the day and $9 \mathrm{~h}$ during the night; total snorkelling: $201 \mathrm{~h}$ during the day and $78 \mathrm{~h}$ during the night) for five years (2014 to 2019). Data from other divers were added whenever pictures allowed the identification of species. Main diving position was around Mayotte Island (Figure 1).

Data presented in this work were not collected following quantitative standards because of the difficulty to identify species while diving and recording all individual observations. I exclusively focused on photographs that allowed reliable species identification at the cost of omitting observations for which no photographs were available or clear enough. Besides literature, websites like WoRMS (Horton et al. 2020), Fishbase (Froese y Pauly 2020), Doris (2020), and MNHN-OFB (2020) were used to get information on the species. Indicators of abundance represented the approximate number of observations relative to the survey effort. The four categories of observation frequency were: 'very rarely', 'rarely', 'commonly' and 'very commonly'. Comparisons of the proportion of species of Muraenidae Family out of all species of fish registered between islands in the Southwestern Indian Ocean, as well as family classification, were based on Fricke et al. (2018).

\section{RESULTS}

A total of 47 species were listed for Mayotte, including 14 species previously unknown from this site (Table 1). Out of all the records, 45 species could be identified to species level. The species assigned to the Genus Anarchias could not be clearly identified based on photographs. A species assigned to the Genus Uropterygius probably represents an undescribed species.

Table 1. Checklist of the Anguilliformes recorded from Mayotte. Species not previously recorded from Mayotte in any publications are in black. TS: this study.

\begin{tabular}{|c|c|c|c|c|c|c|c|c|c|}
\hline & Family & Species & $\begin{array}{l}\text { Böhlke } \\
\text { and } \\
\text { Randall } \\
(2000)\end{array}$ & $\begin{array}{l}\text { Deliot Fouquet } \\
(2000)(2000)\end{array}$ & $\begin{array}{l}\text { Keith } \\
\text { et al. } \\
(2006)\end{array}$ & $\begin{array}{l}\text { Wickel } \\
\text { and } \\
\text { Jamon } \\
(2010)\end{array}$ & $\begin{array}{l}\text { Wickel } \\
\text { et al. } \\
(2014)\end{array}$ & $\begin{array}{l}\text { Allaria } \\
(2016)\end{array}$ & TS \\
\hline 1 & Anguillidae & Anguilla bicolor bicolor & & & 1 & & 1 & & 1 \\
\hline 2 & & Anguilla marmorata & & & 1 & & 1 & & 1 \\
\hline 3 & & Anguilla mossambica & & & 1 & & 1 & & \\
\hline 4 & Colocongridae & Coloconger scholesi & & & & 1 & 1 & & \\
\hline 5 & Congridae & Conger cinereus & & & & & 1 & & 1 \\
\hline 6 & & Heteroconger hassi & & & & & 1 & 1 & 1 \\
\hline 7 & Moringuidae & Moringua macrochir & & & & & & & 1 \\
\hline 8 & & Moringua microchir & & & & & 1 & & 1 \\
\hline 9 & Muraenesocidae & Muraenesox bagio & & 1 & & 1 & 1 & & \\
\hline 10 & Muraenidae & Anarchias sp. & & & & & & & 1 \\
\hline 11 & & Echidna nebulosa & & & & 1 & 1 & 1 & 1 \\
\hline 12 & & Echidna polyzona & & & & & & & 1 \\
\hline 13 & & Enchelycore pardalis & & & & & & 1 & 1 \\
\hline
\end{tabular}


Table 1. Continued.

\begin{tabular}{|c|c|c|c|c|c|c|c|c|c|}
\hline Family & Species & $\begin{array}{l}\text { Böhlke } \\
\text { and } \\
\text { Randall } \\
(2000)\end{array}$ & $\begin{array}{l}\text { Deliot } \\
(2000)\end{array}$ & $\begin{array}{c}\text { Fouquet } \\
(2000)\end{array}$ & $\begin{array}{l}\text { Keith } \\
\text { et al. } \\
(2006)\end{array}$ & $\begin{array}{l}\text { Wickel } \\
\text { and } \\
\text { Jamon } \\
(2010)\end{array}$ & $\begin{array}{c}\text { Wickel } \\
\text { et al. } \\
\text { (2014) }\end{array}$ & $\begin{array}{l}\text { Allaria } \\
(2016)\end{array}$ & $\mathrm{TS}$ \\
\hline 14 & Gymnomuraena zebra & & & & & 1 & 1 & 1 & 1 \\
\hline 15 & Gymnothorax breedeni & & & & & 1 & 1 & 1 & 1 \\
\hline 16 & Gymnothorax chilospilus & & & & & & & & 1 \\
\hline 17 & Gymnothorax favagineus & & & & & 1 & 1 & 1 & 1 \\
\hline 18 & Gymnothorax fimbriatus & & & & & & & & 1 \\
\hline 19 & Gymnothorax flavimarginatus & & & & & & 1 & 1 & 1 \\
\hline 20 & Gymnothorax griseus & & & & & & 1 & & 1 \\
\hline 21 & Gymnothorax javanicus & & & & & 1 & 1 & 1 & 1 \\
\hline 22 & Gymnothorax melatremus & & & & & & & & 1 \\
\hline 23 & Gymnothorax meleagris & & & & & 1 & 1 & 1 & 1 \\
\hline 24 & Gymnothorax nudivomer & & & & & & & 1 & 1 \\
\hline 25 & Gymnothorax phasmatodes & & & & & & & & 1 \\
\hline 26 & Gymnothorax pictus & & & & & 1 & 1 & & 1 \\
\hline 27 & Gymnothorax richardsonii & & & & & & & & 1 \\
\hline 28 & Gymnothorax rueppelliae & 1 & & & & & & & 1 \\
\hline 29 & Gymnothorax undulatus & & & & & & & 1 & 1 \\
\hline 30 & Gymnothorax zonipectis & & & & & & & 1 & 1 \\
\hline 31 & Pseudechidna brummeri & & & & & 1 & 1 & & 1 \\
\hline 32 & Rhinomuraena quaesita & & & & & 1 & 1 & 1 & 1 \\
\hline 33 & Scuticaria tigrina & & & & & & & 1 & 1 \\
\hline 34 & Strophidon sathete & & 1 & & & 1 & 1 & & \\
\hline 35 & Uropterygius micropterus & & & & & & & & 1 \\
\hline 36 & Uropterygius nagoensis & & & & & & & & 1 \\
\hline 37 & Uropterygius polyspilus & 1 & & & & & & & 1 \\
\hline 38 & Uropterygius sp. & & & & & & & & 1 \\
\hline 39 Ophichthidae & Brachysomophis crocodilinus & & & & & & & & 1 \\
\hline 40 & Brachysomophis henshawi & & & & & & & 1 & \\
\hline 41 & Callechelys marmorata & & & & & & & 1 & 1 \\
\hline 42 & Myrichthys colubrinus & & & & & 1 & 1 & 1 & 1 \\
\hline 43 & Myrichthys maculosus & & & & & & 1 & & 1 \\
\hline 44 & Ophichthus altipennis & & & & & & & & 1 \\
\hline 45 & Ophichthus bonaparti & & & 1 & & 1 & 1 & & 1 \\
\hline 46 & Pisodonophis cancrivorus & & & & & & & & 1 \\
\hline 47 Synaphobranchidae & Meadia abyssalis & & & & & 1 & & & \\
\hline
\end{tabular}


The largest family was represented by the Muraenidae with 29 species (62\% of the total, Figure 2) followed by Ophichtidae (8 species, $17 \%$ ). Muraenidae species found in this study represented $3.82 \%$ of the total number of fish species of the lagoon of Mayotte reported by Wickel et al. (2014) (Table 2).

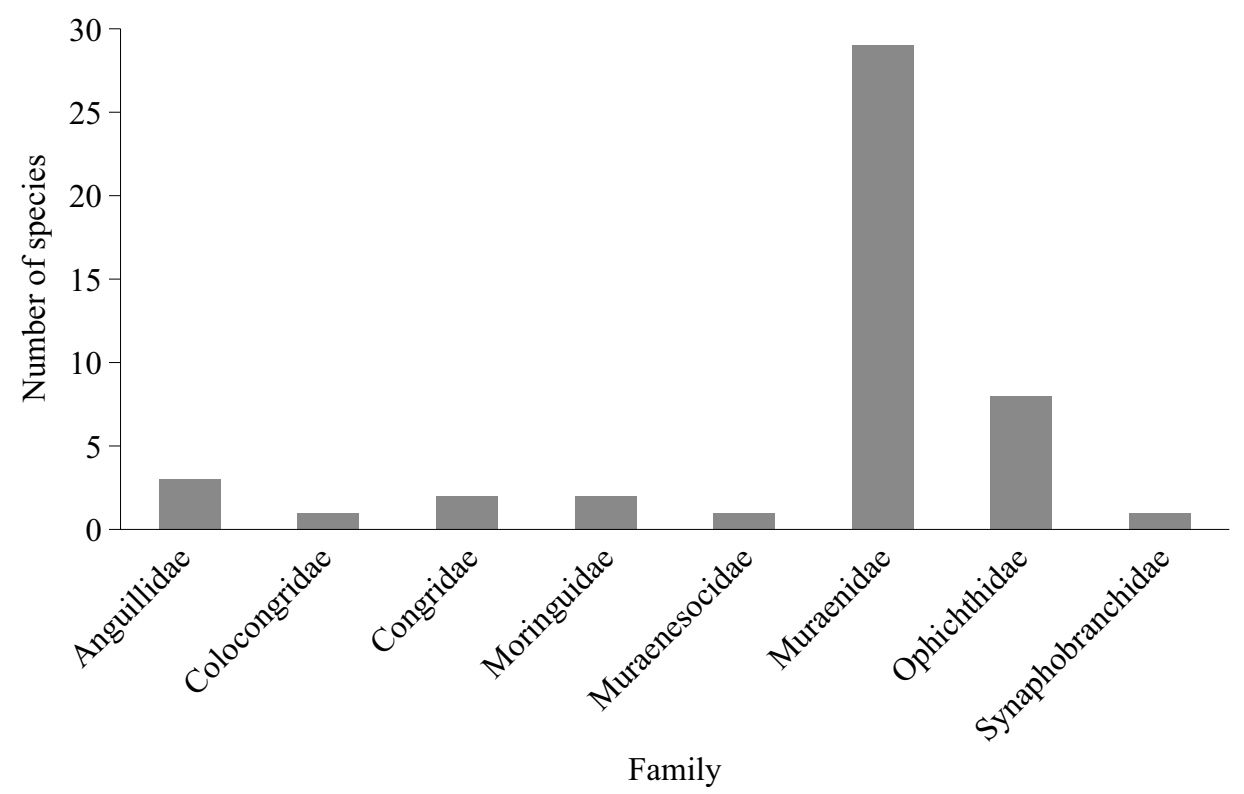

Figure 2. Number of species for each family of Anguilliformes recorded.

Table 2. Comparison of the proportion of Muraenidae species from Mayotte lagoon and other islands in the Indian Ocean (Fricke et al. 2018).

\begin{tabular}{lcc}
\hline & $\begin{array}{c}\text { Proportion of } \\
\text { Muraenidae }\end{array}$ & $\begin{array}{c}\text { Total species number } \\
\text { of all fish }\end{array}$ \\
\hline Madagascar & 2.7 & 1,365 \\
Europa Island & 2.3 & 389 \\
Seychelles & 2.9 & 917 \\
Maldives & 4 & 1,022 \\
Chagos Archipelago & 5.4 & 782 \\
Réunion & 3.8 & 835 \\
Mauritius & 3.9 & 945 \\
Rodrigues & 2.6 & 495 \\
Mayotte & $3.8^{*}$ & $759 * *$ \\
\hline
\end{tabular}

* Proportion (percent of total species number) of Muraenidae found in this study compared to the total of fish species found in Wickel et al. (2014).

**Wickel et al. (2014). 


\section{Class Actinopterygii \\ Order Anguilliformes \\ Family Anguillidae}

Anguilla bicolor bicolor McClelland, 1844

Indonesian shortfin eel (EN);

Anguille bicolore (FR) (Figure 3)

Status at Mayotte. First record from Mayotte by G. Amirault, M. Lamalfa Diaz, and O. Soumille (from 2003 to 2018, ZNIEFF inventories -Zone Naturelle d'Intérêt Ecologique, Faunistique et Floristique) INPN (Inventaire National du Patrimoine Naturel); subsequently reported from Mayotte by Keith et al. (2006) and by Wickel et al. (2014).

Other Observations. Observed very rarely. One observation in 2017 by Yannick Stefan in Sohoa creek close to Sohoa beach $\left(12^{\circ} 49^{\prime} 10.3^{\prime \prime} \mathrm{S}-45^{\circ}\right.$ 06' 22.1" E) about $50 \mathrm{~m}$ depth.

Description. The back is brown-green and the belly light gray or yellowish. It becomes silver during catadrome migration. Nostrils are yellow. It does not exceed $1.2 \mathrm{~m}$ total length (TL). Pectoral fins are well developed. Dorsal fin short, originating a little anterior from the anus (Keith et al. 2006).
Distribution and ecology. Wide range across the Indian Ocean. Found on the African cost (from Kenya to South Africa), Madagascar, the Comoros Islands (except Ngazidja), Mauritius, Rodrigues, Seychelles, India and Malaysia (Keith et al. 2006). The subspecies A. bicolor pacifica is found in the Pacific. Indonesia marks the biogeographic boundary between these two subspecies (Fahmi 2015). It lives in running and stagnant bodies of freshwater, feeding at night on fish, crustaceans and molluscs. Catadromous species.

Anguilla marmorata Quoy and Gaimard, 1824 Giant mottled eel (EN); Anguille marbrée (FR) (Figure 4)

Status at Mayotte. First record from Mayotte by G. Amirault, M. Lamalfa Diaz, and O. Soumille (from 2003 to 2018, ZNIEFF inventories INPN); subsequently reported from Mayotte by Keith et al. (2006) and by Wickel et al. (2014).

Observation by the author. Observed commonly. E.g.: March 2016, in the lagoon $\left(12^{\circ} 49^{\prime} 45.7^{\prime \prime}\right.$ S$\left.45^{\circ} 17^{\prime} 02.8^{\prime \prime} \mathrm{E}\right)$ by night near the surface; April 2018 in the hilltop reserve of Combani $\left(12^{\circ} 46^{\prime}\right.$ $38.3^{\prime \prime} \mathrm{S}-45^{\circ} 08^{\prime} 32.5^{\prime \prime}$ E) at daytime; November 2018 in Koualé River $\left(12^{\circ} 48^{\prime} 15.0^{\prime \prime}\right.$ S-45 09' $\left.42.5^{\prime \prime} \mathrm{E}\right)$ in muddy running water at night; May
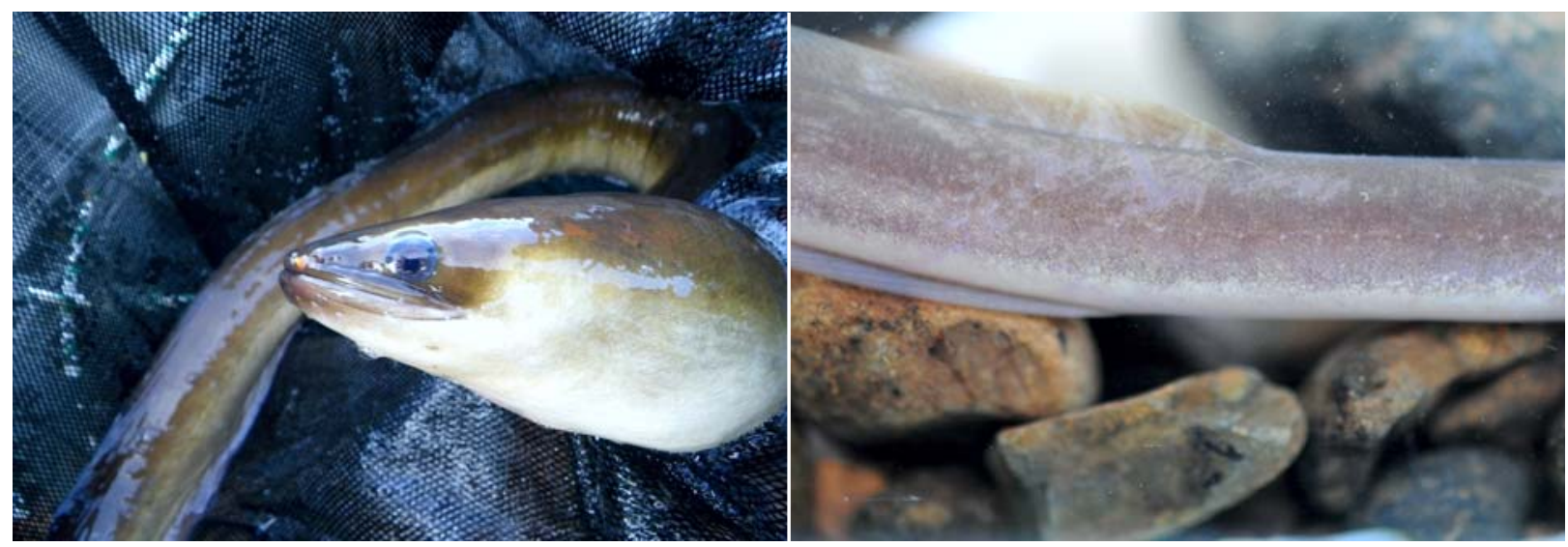

Figure 3. Anguilla bicolor bicolor close up of the head (left) and fins (right) (OCEA Consult' (C), Pierre Valade). 

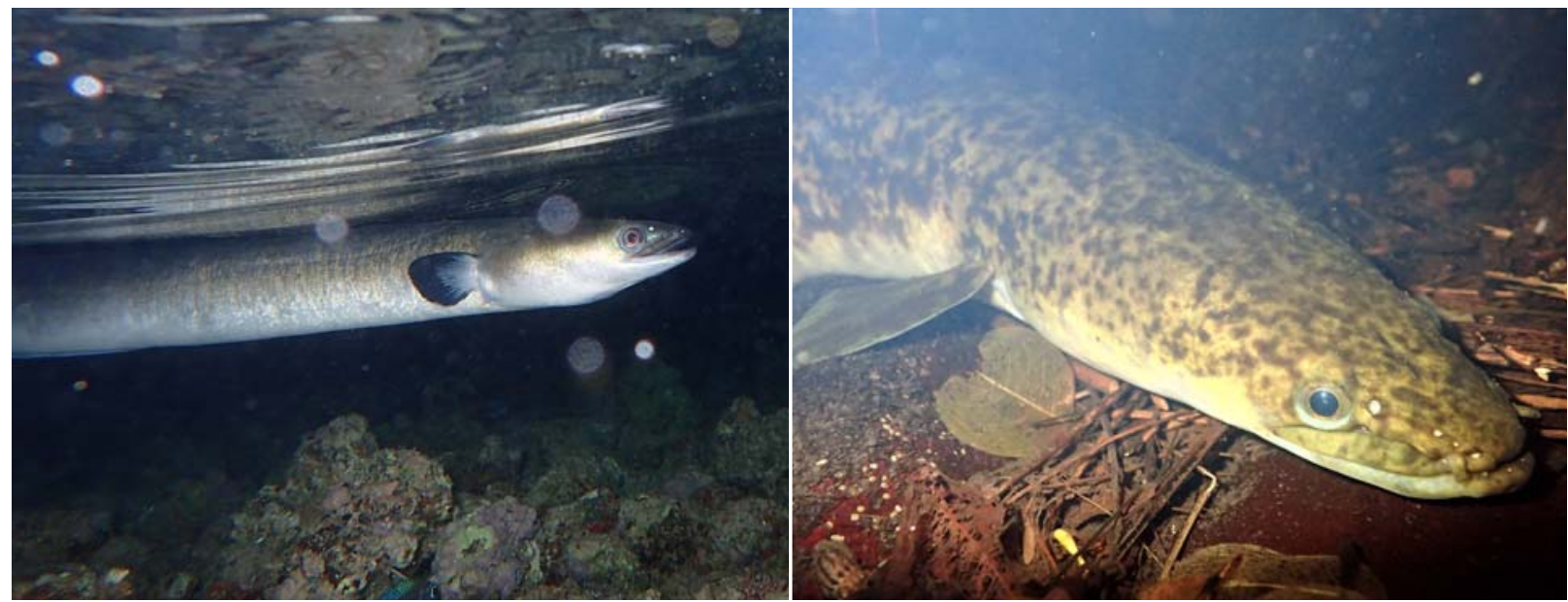

Figure 4. Left: mature individual of Anguilla marmorata. The silver color indicates the migration into the Mayotte Lagoon (12 49' 45.7" S-45 17'02.8" E; 11 th March 2016; 00 h 43 min; surface). Right: A. marmorata mottled color in Koualé River, Vahibé (12॰ 48' 15.0" S- 45 09' 42.5" E; 10th November 2018; 20 h 11 min; depth: 40 cm).

2019 in Bouyouni River (12 44' $19.3^{\prime \prime} \mathrm{S}-45^{\circ} 08^{\prime}$ $24.1^{\prime \prime}$ E) at daytime.

Description. The back is marbled brown-black on a gray-yellow background. The color changes to silver during maturation. The belly is whitish. Females do not exceed $2 \mathrm{~m}$ TL while males reach a maximum of only $70 \mathrm{~cm}$ TL. Pectoral fins are well developed. Dorsal fin long, originating several centimeters anterior from the anus (Keith et al. 2006).

Distribution and ecology. Present throughout the indo-pacific zone from East Africa to French Polynesia, across Indonesia. It lives in running and stagnant bodies of freshwater, feeding at night mainly on shrimps but also fish, insects, molluscs and annelids. Catadromous species.

Anguilla mossambica (Peters, 1852)

African longfin eel (EN);

Anguille du Mozambique (FR) (Figure 5)

Status at Mayotte. First record from Mayotte by G. Amirault, M. Lamalfa Diaz, and O. Soumille (from 2003 to 2006, ZNIEFF inventories INPN);

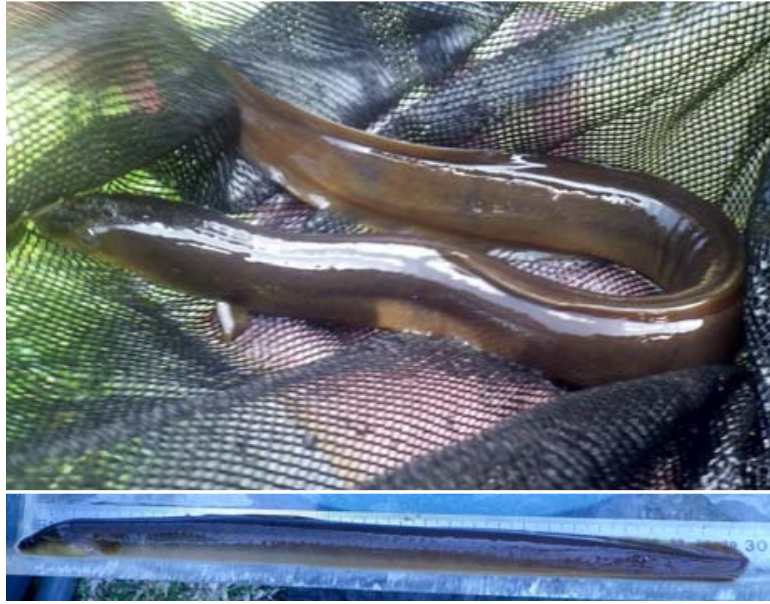

Figure 5. Anguilla mossambica caught for a study of OCEA Consult' (C).

subsequently reported from Mayotte by Keith et al. (2006) and by Wickel et al. (2014).

Observation by the author. No observation during this study. Observed very rarely.

Description. Uniform color with a gray to olivegreen back and a light belly. Black back and silver belly typical for large individuals. Does not 
exceed $1.2 \mathrm{~m}$ TL. Pectoral fins are well developed. Dorsal fin long, originating several centimeters anterior from the anus (Keith et al. 2006).

Distribution and ecology. Range limited to the Westerm Indian Ocean. Found in Kenya, South Africa, Madagascar, the Comoros Islands (except Ngazidja), Mauritius and La Réunion (Keith et al. 2006). It lives in running and stagnant bodies of freshwater, feeding at night on fish and crustaceans. Catadromous species.

\section{Family Colocongridae}

Coloconger scholesi Chan, 1967

Indo-Pacific shorttail conger (EN);

Congre à queue courte (FR) (Figure 6)

Status at Mayotte. First record from Mayotte by Wickel and Jamon (2010); subsequently reported from Mayotte by Wickel et al. (2014).

Observation by the author. No observation during this study. Observed very rarely.

Description. Small eel, dark greyish to blackishbrown with a very short tail. Short head. Pectoral fins are well developed. Origin of the dorsal fin at

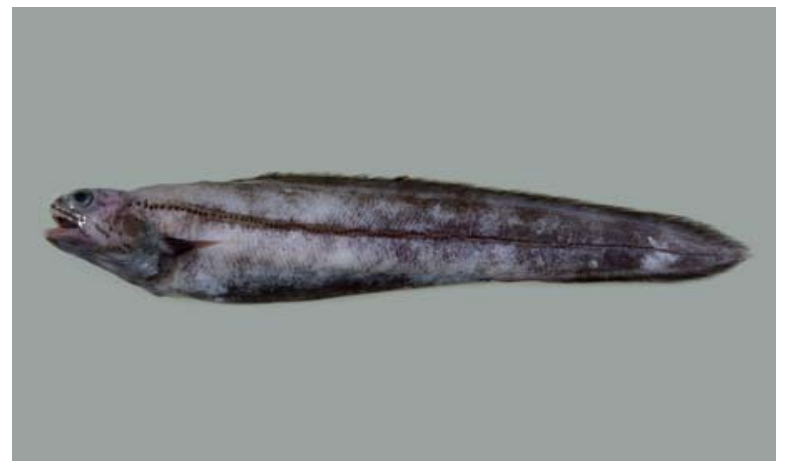

Figure 6. A shorttail conger, Coloconger scholesi, from off northwestern Western Australia, CSIRO H 6577-02. Source: Australian National Fish Collection, CSIRO. License: CC BY Attribution-Noncommercial. Taken from Bray (2020a). the same level as the base of the pectoral fin (Karrer 1982). Maximum length $51 \mathrm{~cm}$ TL (Castle 1986).

Distribution and ecology. Indo-pacific distribution. Found in waters off Southern Mozambique, Madagascar, South Africa, Australia and South China (Fricke et al. 2018). Inhabits deep waters from 412 to $970 \mathrm{~m}$ (Fricke et al. 2011).

\section{Family Congridae}

Conger cinereus Rüppell, 1830

Longfin African conger (EN);

Congre à moustache (FR) (Figure 7)

Status at Mayotte. First record from Mayotte by Wickel et al. (2014).

Observation by the author. Observed commonly. For example: July 2016 on Mzouazia bay seagrass beds ( $\left.12^{\circ} 56^{\prime} 50.9^{\prime \prime} \mathrm{S}-45^{\circ} 05^{\prime} 53.2^{\prime \prime} \mathrm{E}\right)$ at night; March 2017 on Tsingoni beach seagrass beds (12 $\left.47^{\prime} 13.0^{\prime \prime} \mathrm{S}-45^{\circ} 05^{\prime} 38.0^{\prime \prime} \mathrm{E}\right)$ at night; April 2017 Mtsanga Mtiti Sada seagrass beds (12 50' 59.0" S-45 05' 26.7" E) at night; June 2017 on Bandrele islet reef flat $\left(12^{\circ} 53^{\prime} 43.3^{\prime \prime} \mathrm{S}\right.$ $\left.45^{\circ} 13^{\prime} 48.1^{\prime \prime} \mathrm{E}\right)$ at night.

Description. Gray to light brown with many gray to blackish vertical bars at night. Blackish oblique band from the lower part of the eye to the corner of the lips. The pectoral fins are well developed. Origin of the dorsal fin over the middle of the pectoral fin (Smith 1999). Maximum length 140 cm (Castle 1986).

Distribution and ecology. Red Sea, East Africa, Seychelles, to Madagascar and Mascarenes east to Japan and Ogasawara Islands, Hawaiian Islands, Line Islands and Pitcairn Group, south to Western Australia, Lord Howe Island, New Caledonia and Rapa (Fricke et al. 2018). Inhabits reef flats and seagrass beds of shallow lagoons but ranges to depths of $80 \mathrm{~m}$ on outer reef slopes 


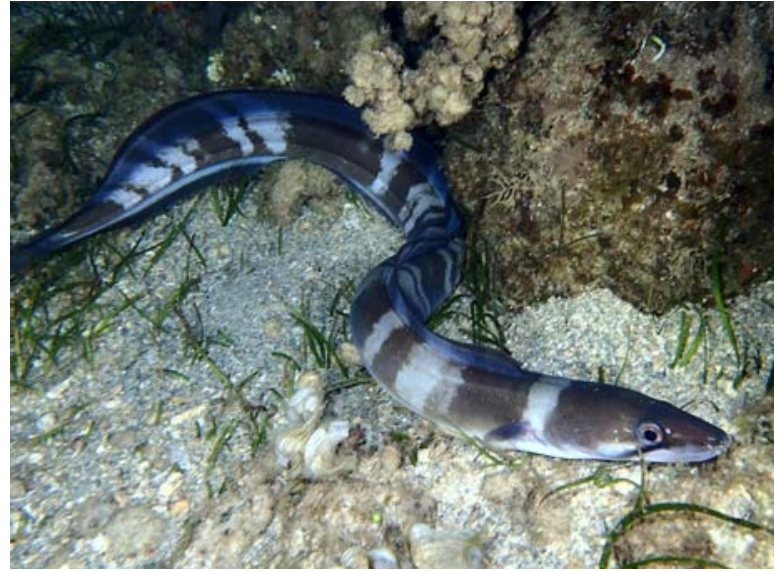

Figure 7. Conger cinereus on seagrass beds at night, Mtsanga Mtiti, Sada ( $12^{\circ} 50^{\prime} 59.0^{\prime \prime} \mathrm{S}-45^{\circ} 05^{\prime} 26.7^{\prime \prime}$ E; 23th April 2017; 21 h 31 min; depth: $1 \mathrm{~m}$ ).

(Myers, 1991). Feeds on fish, crustaceans, and other invertebrates, which it hunts at night.

\section{Heteroconger hassi}

(Klausewitz and Eibl-Eibesfeldt, 1959)

Spotted Garden Eel (EN);

Hétérocongre tacheté (FR) (Figure 8)

Status at Mayotte. First record from Mayotte by Letourneur and Maggiorani (1995); subsequently reported from Mayotte by Wickel et al. (2014) and Allaria (2016).

Observation by the author. Observed commonly. E.g.: December 2016 at Passe en S (12 52' 17.5" S-45 $\left.16^{\prime} 25.8^{\prime \prime} \mathrm{E}\right)$ at daytime; April 2017 at Passe Bateau (12 $\left.58^{\prime} 39.0^{\prime \prime} \mathrm{S}-44^{\circ} 59^{\prime} 08.4^{\prime \prime} \mathrm{E}\right)$ at daytime; March 2018 at Passe en S (12 52' 05.5" S$45^{\circ} 16^{\prime} 23.5^{\prime \prime}$ E) at daytime; November 2018 at Boa sand banc $\left(12^{\circ} 41^{\prime} 10.7^{\prime \prime} \mathrm{S}-45^{\circ} 02^{\prime} 18.1^{\prime \prime} \mathrm{E}\right)$ at daytime.

Description. Small white eel with variable black spots and usually two large black spots on each flank. Very small pectoral fins. Maximum size 70 cm TL (Allen et al. 2003).

Distribution and ecology. East Africa, Seychelles, Comoros, Madagascar and Mascarenes, Tonga, Samoa and Line Islands, north to Ryukyu Islands and Ogasawara Islands, Taiwan, Australia and New Caledonia (Fricke et al. 2018). Form large colonies on sandy bottom in the lagoon and around seaward reefs between 5 and $50 \mathrm{~m}$ depth (Allen et al. 2003). Withdraw into their burrow when approached. Feed on zooplankton organisms caught in the current (Bacchet et al. 2006).

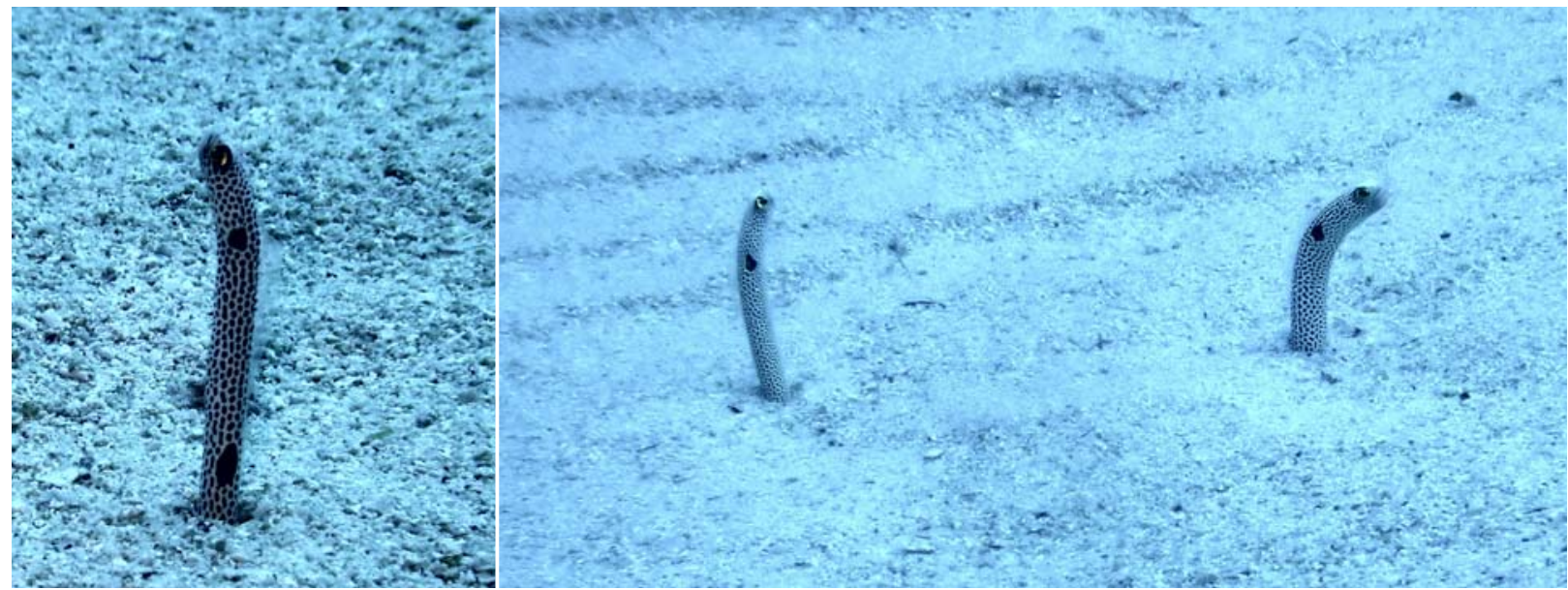

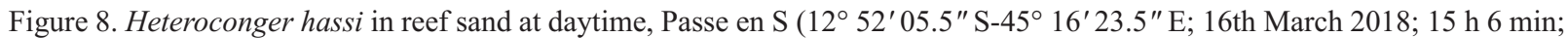
depth: $18 \mathrm{~m})$. 


\section{Family Moringuidae}

Moringua macrochir Bleeker, 1853

Longfin spaghetti eel (EN) (Figure 9)

Status at Mayotte. No record prior to this study.

Observation by divers. Observed rarely. By Norbert Verneau on Papani reef flat at night.

Description. Long and tapered eel. Big eyes compared to the body. Brownish dorsally, gradually fading to white ventrally. About 69 pores on the lateral line anterior to the anus. Maximum size 20 cm TL (Allen and Erdmann 2012).

Distribution and ecology. Indo-West Pacific (Christmas Island, Philippines and Indonesia). East Africa and Madagascar east to the Phoenix and Line Islands (Fricke et al. 2018). Occur in brackish and marine waters (Fricke et al. 2018) to a maximum depth of $15 \mathrm{~m}$ (Fricke et al. 2011).

Moringua microchir Bleeker, 1853

Lesser thrush eel (EN); Anguille spaghetti (FR) (Figure 10)

Status at Mayotte. First record from Mayotte by Wickel et al. (2014).

Observation by divers. Observed commonly (around 10 times) by N. Verneau on Papani reef flat at night while moving blocks of rock or coral.

Description. Long and tapered eel. Adults all brown on the back and lighter on the belly. Small pectoral fins. Juveniles have an orange-yellow back and a bluish belly. Maximum size $47 \mathrm{~cm}$ TL (Keith et al. 2006).

Distribution and ecology. South and East Africa, Seychelles, Madagascar and Mauritius east to Samoa, north to Ryukyu Islands, south Great Barrier Reef (Australia) and New Caledonia (Fricke

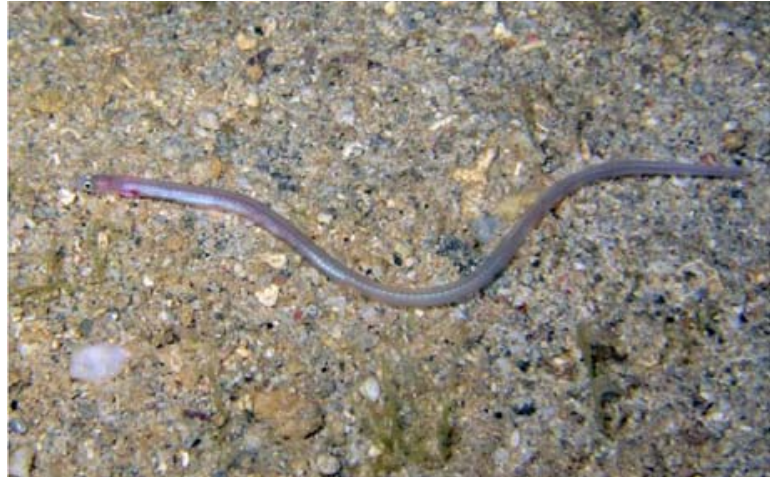

Figure 9. Moringua macrochir on reef flat, Papani (at night; $2 \mathrm{~m}$ ). Reproduce with permission from Norbert Verneau.

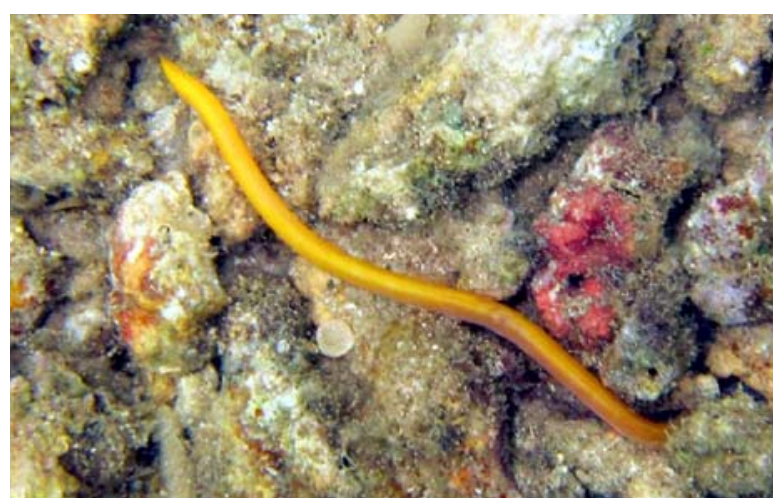

Figure 10. Moringua microchir on reef flat, Papani $\left(12^{\circ} 45^{\prime}\right.$ 31.6" S-45 $17^{\prime} 24.1^{\prime \prime}$ E; 17th December 2010; 22 h $26 \mathrm{~min} ; 1 \mathrm{~m}$ depth). Reproduced with permission from Norbert Verneau.

et al. 2018). Occur in estuaries, lower parts of rivers and sandy bottoms where they eat fish and crustaceans (Keith et al. 2006).

\section{Family Muraenesocidae}

Muraenesox bagio (Hamilton, 1822)

Common pike conger (EN);

Morénésoce commun (FR) (Figure 11)

Status at Mayotte. First record from Mayotte by Wickel and Jamon (2010); subsequently reported from Mayotte by Wickel et al. (2014). 


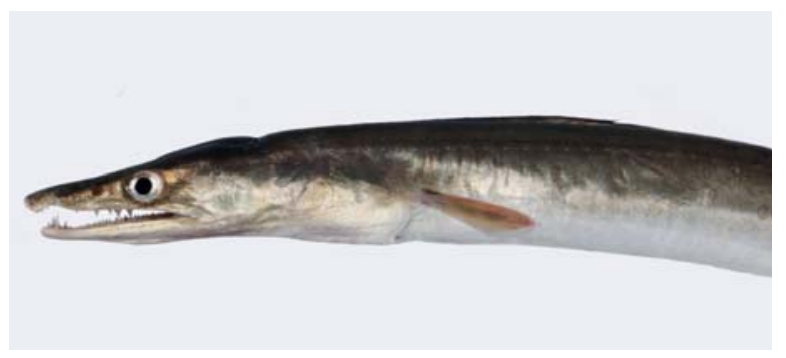

Figure 11. Head of a Common Pike Eel, Muraenesox bagio, from Pilar Public Market, Sorsogon Province, Luzon, Philippines - USNM 405904, $654 \mathrm{~mm}$ TL. Source: Jeffrey T. Williams / Smithsonian Institution, National Museum of Natural History. License: CC BY Attribution-Noncommercial-ShareAlike 3.0. Taken from Bray (2020b).

Observation by the author. No observation during this study. Observed very rarely.

Description. Long silvery eel. Pectoral fins are well developed. Narrow head. Very large mouth with conspicuous teeth (Talwar et al. 1991). Maximum size $200 \mathrm{~cm}$ TL (Masuda et al. 1984).

Distribution and ecology. South Africa, East Africa, Madagascar and western Mascarenes east of Samoa, Yellow Sea, Kuril Islands, Australia and New Caledonia (Fricke et al. 2018). Found in coastal waters, including estuaries, above $100 \mathrm{~m}$ depth (Yamada et al. 1995). Feed on benthic fishes and crustaceans at night (Smith 1997).

\section{Family Muraenidae}

Anarchias sp. Jordan and Starks, 1906

(Figure 12)

Photography does not allow unequivocal identification to the species level, but Anarchias seychellensis Smith, 1962 is the most likely candidate. Coloration also resembles of Anarchias galapagensis. I therefore decided to keep the species as Anarchias sp. The information given on the description, distribution, and ecology are those of $A$. seychellensis.

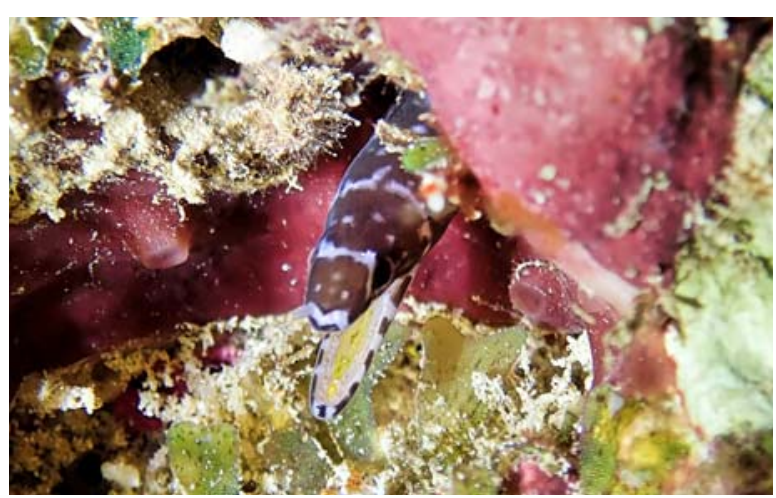

Figure 12. Anarchias sp. in March 2019, Passe en S (at night, depth: $10 \mathrm{~m})$. Reproduced with permission from Frederic Ducarme.

Status at Mayotte. No record prior to this study.

Observation by divers. Observed very rarely. One observation by Frederic Ducarme in March 2019, at Passe en S, at night.

Description. Very small moray, slender. Front nostrils tubular and pointing forward. Presence of a row of irregular or stellate pale spots. Size around $30 \mathrm{~cm}$ TL.

Distribution and ecology. East and South Africa, Seychelles, Comoros, Madagascar and Réunion Island, east to Hawaiian Islands and Easter Island, north to Ogasawara Islands, south to Western Australia, New Caledonia, New Zealand and Kermadec Islands (Fricke et al. 2018). Found among rubble, coralline algal debris, and coral heads of shallow wave-washed reefs (Myers 1991) between 0-35 $\mathrm{m}$ depth (Fricke et al. 2011).

Echidna nebulosa (Ahl, 1789)

Snowflake moray (EN); Murène étoilée (FR)

(Figure 13)

Status at Mayotte. First record from Mayotte by Wickel and Jamon (2010). Subsequently reported from Mayotte by Wickel et al. (2014) and by Allaria (2016). 


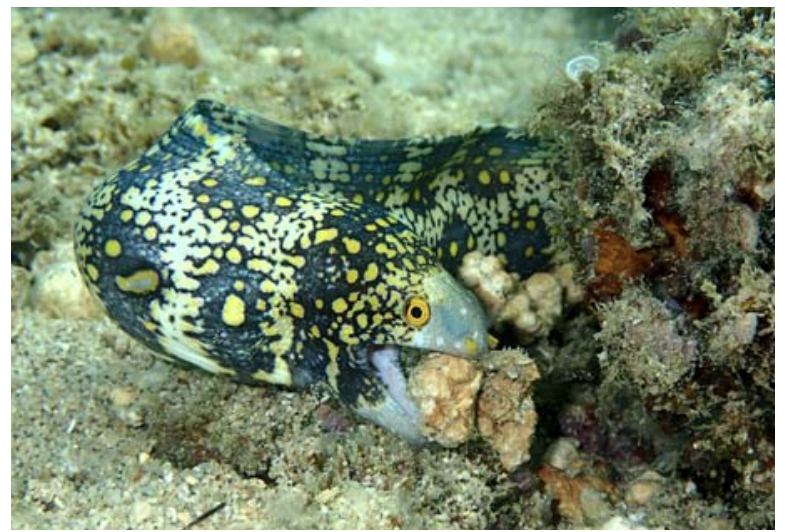

Figure 13. Echidna nebulosa on reef flat, Bandrele islet $\left(12^{\circ}\right.$ 53' 40.3" S-45 13' 50.9" E; 3rd March 2018; 14 h $27 \mathrm{~min} ; 1.5 \mathrm{~m}$ depth).

Observation by the author. Observed very commonly. E.g.: June 2017 near Bandrele islet $\left(12^{\circ}\right.$ $\left.53^{\prime} 40.7^{\prime \prime} \mathrm{S}-45^{\circ} 13^{\prime} 56.1^{\prime \prime} \mathrm{E}\right)$ at daytime; October 2017 near Sada islet $\left(12^{\circ} 50^{\prime} 47.9^{\prime \prime} \mathrm{S}-45^{\circ} 05^{\prime}\right.$ 17.2" E) at daytime; November 2017 Sakouli beach flat reef $\left(12^{\circ} 53^{\prime} 19.1^{\prime \prime} \mathrm{S}-45^{\circ} 12^{\prime} 53.7^{\prime \prime} \mathrm{E}\right)$ at night; March 2018 near Bandrele islet $\left(12^{\circ} 53^{\prime}\right.$ $\left.40.3^{\prime \prime} \mathrm{S}-45^{\circ} 13^{\prime} 50.9^{\prime \prime} \mathrm{E}\right)$ at daytime.

Description. Small, light gray to brown moray finely reticulated with black. Two longitudinalrows of large black blotches containing yellow spots. Iris and anterior nostrils yellow (Smith et al. 2019). Maximum size $75 \mathrm{~cm}$ TL (Allen et al. 2003).

Distribution and ecology. Red Sea and IndoPacific. East Africa, South Africa, Seychelles, Comoros, Madagascar and Mascarenes east to Hawaiian Islands and Panama, north to Ryukyu Islands, Ogasawara Islands and Johnston Atoll, south to Western Australia and New Caledonia (Fricke et al. 2018). Found in reef flats and rocky shorlines (Allen et al. 2003) sometimes in seagrass areas, usually from depths less than $3 \mathrm{~m}$, but also reported from $48 \mathrm{~m}$ depth (Smith et al. 2019). Feed mainly on small crustaceans at night but also easily observed during the day.
Echidna polyzona (Richardson, 1845)

Barred Moray (EN); Murène à barres (FR) (Figure 14)

Status at Mayotte. No record prior to this study.

Observation by the author. Observed rarely. Three times: March 2015 at Sakouli beach reef flat $\left(12^{\circ} 53^{\prime} 15.8^{\prime \prime} \mathrm{S}-45^{\circ} 12^{\prime} 55.9^{\prime \prime} \mathrm{E}\right)$ at night; May 2017 near Gombé Ndroumé islet $\left(12^{\circ} 45^{\prime}\right.$ $\left.00.3^{\prime \prime} \mathrm{S}-45^{\circ} 16^{\prime} 13.2^{\prime \prime} \mathrm{E}\right)$ at night; December 2018 at Sakouli beach reef flat $\left(12^{\circ} 53^{\prime} 15.8^{\prime \prime} \mathrm{S}-45^{\circ} 12^{\prime}\right.$ 55.9" E) at night.

Description. Small moray, alternating broad dark brown and narrow white bars on head and body. Corner of the mouth is dark. Anterior nostrils brownish yellow (Smith et al. 2019). Maximum size $72 \mathrm{~cm}$ TL (Allen and Erdmann 2012).

Distribution and ecology. Red Sea, East and South Africa, Seychelles, Madagascar and Mascarenes east to Marshall and Tuamotu Islands, Japan and Taiwan, Australia and New Caledonia (Fricke et al. 2018). Found in reef flats, clear shallow lagoons, and seaward reefs between 1 to $20 \mathrm{~m}$ depth. Feed mainly on small crustaceans during both day and night (Lieske and Myers 2005).

\section{Enchelycore pardalis}

(Temminck and Schlegel, 1846)

Leopard moray eel (EN); Murène dragon (FR)

(Figure 15)

Status at Mayotte. First record from Mayotte by Allaria (2016).

Observation by the author. Observed rarely. Few times at the same spot. January 2018 at Passe des îles Choizil ( $\left.12^{\circ} 42^{\prime} 59.9^{\prime \prime} \mathrm{S}-44^{\circ} 59^{\prime} 19.0^{\prime \prime} \mathrm{E}\right)$ at daytime.

Description. Pale yellow to orange with many white and some dark spots. Curved jaws with 

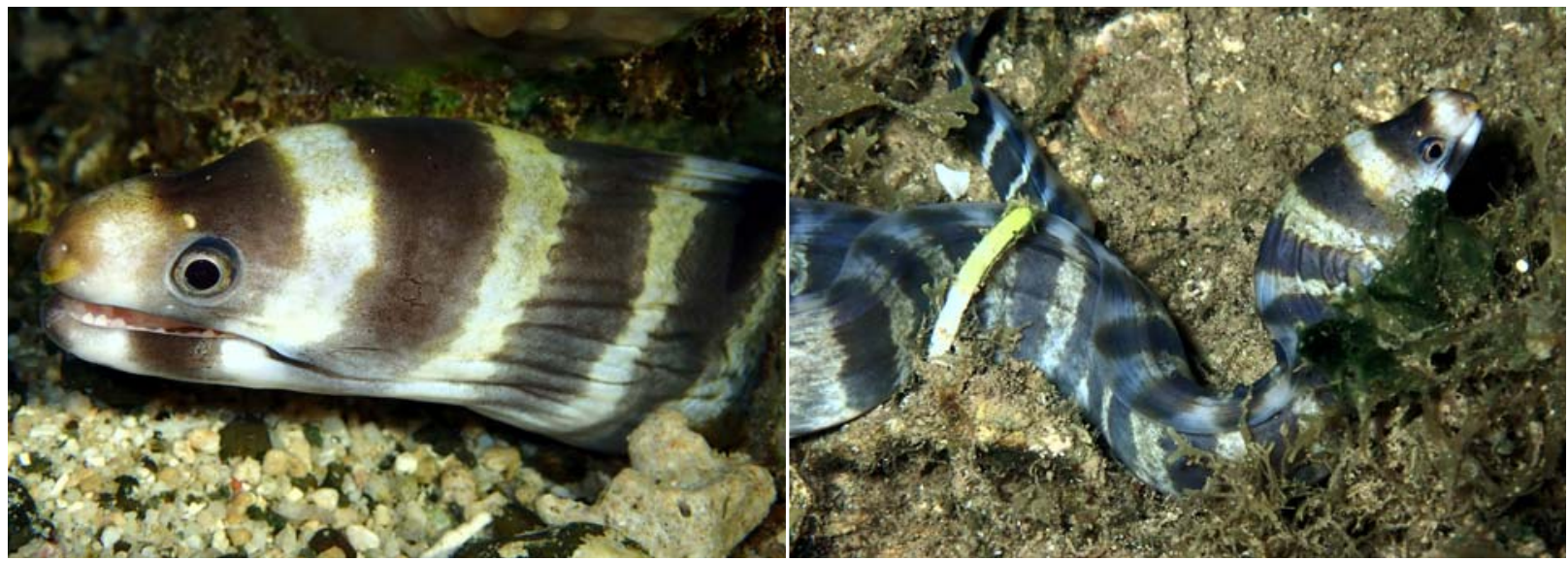

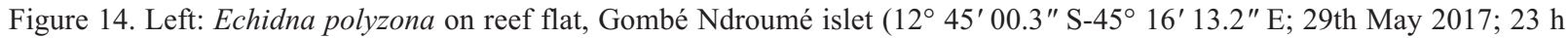

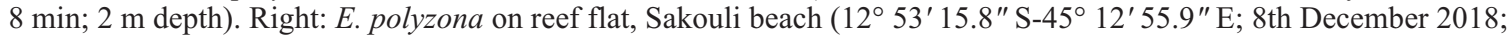
23 h 59 min; 1 m depth).

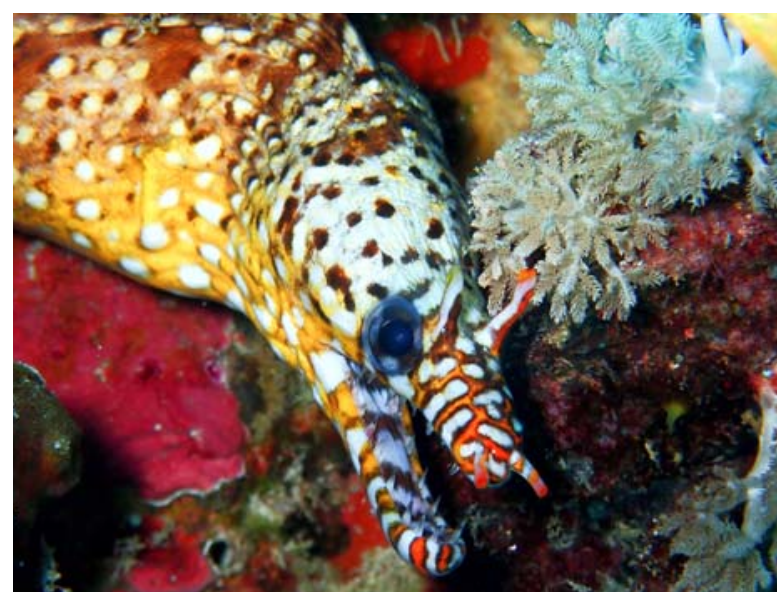

Figure 15. Enchelycore pardalis in reef boulder around sandy bottom, Passe des îles Choizil $\left(12^{\circ} 42^{\prime} 59.9^{\prime \prime} \mathrm{S}\right.$ $44^{\circ} 59^{\prime} 19.0^{\prime \prime} \mathrm{E}$; 19th January 2018; 14 h 49 min; 18 $\mathrm{m}$ depth).

many sharp teeth. Long tubular nostrils above the eyes (Allen et al. 2003). Maximum size $92 \mathrm{~cm}$ TL (Randall 2005).

Distributionand ecology. Aldabra, Madagascar and Mascarenes east to Hawaiian, Line and Marquesas islands, north to Korea, southern Japan and Ogasawara Islands, south to New Caledonia (Fricke et al. 2018). Found in coral reefs and rocky areas (Lieske and Myers 2005) between 15 and $50 \mathrm{~m}$ depth (Allen et al. 2003). Feed on fishes (Lieske and Myers 2005).

Gymnomuraena zebra (Shaw, 1797)

Zebra Moray (EN); Murène zèbrée (FR) (Figure 16)

Status at Mayotte. First record from Mayotte by Wickel and Jamon (2010). Subsequently reported from Mayotte by Wickel et al. (2014) and Allaria (2016).

Observation by the author. Observed commonly. E.g.: May 2015 near Handréma islet $\left(12^{\circ} 40^{\prime}\right.$ 26.2" S-45 07' 07.8" E) at daytime; October 2015 near White sand islet $\left(12^{\circ} 59^{\prime} 08.5^{\prime \prime} \mathrm{S}-45^{\circ}\right.$ $\left.12^{\prime} 54.0^{\prime \prime} \mathrm{E}\right)$ at daytime; July 2016 near Bandrélé islet (12 $\left.{ }^{\circ} 54^{\prime} 03.0^{\prime \prime} \mathrm{S}-45^{\circ} 13^{\prime} 59.3^{\prime \prime} \mathrm{E}\right)$ at daytime; May 2018 at Tsingoni beach reef $\left(12^{\circ} 47^{\prime} 13.6^{\prime \prime} \mathrm{S}-\right.$ $45^{\circ} 05^{\prime}$ 29.7" E) at night; August 2018 at Mtsongoma islet reef $\left(12^{\circ} 41^{\prime} 17.9^{\prime \prime} \mathrm{S}-45^{\circ} 07^{\prime}\right.$ $40.9^{\prime \prime}$ E) at daytime.

Description. Small but chunky moray. Dark brown to chocolate in color, with narrow pale yellowish or white bars on the head and body (Smith 


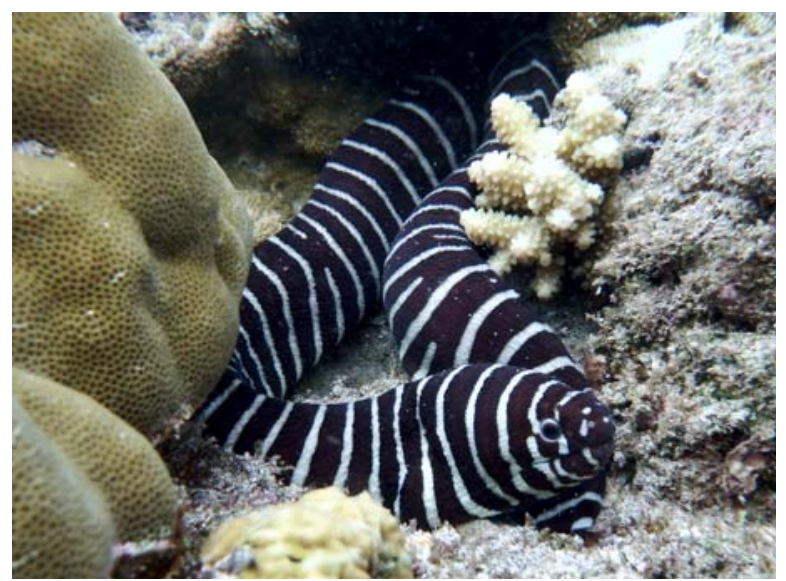

Figure 16. Gymnomuraena zebra on coral reef, Handréma islet $\left(12^{\circ} 40^{\prime} 26.2^{\prime \prime} \mathrm{S}-45^{\circ} 07^{\prime} 07.8^{\prime \prime} \mathrm{E}\right.$; 10th May 2015; 12 h $30 \mathrm{~min} ; 2.5 \mathrm{~m}$ depth).

et al. 2019). Short nose with pale anterior nostrils. Maximum size $150 \mathrm{~cm}$ TL (Lieske and Myers 2005).

Distribution and ecology. East Africa, Seychelles, Madagascar and Mascarenes east to Hawaiian Islands, Galápagos Archipelago, and Revillagigedo Archipelago, north to Ryukyu Islands and Ogasawara Islands, south to Western Australia and New Caledonia (Fricke et al. 2018). Found in shallow water and on coral reefs at depths of 1-50 $\mathrm{m}$ (Smith et al. 2019). Feed on crustaceans, molluscs and sea urchin (Lieske and Myers 2005).

\section{Gymnothorax breedeni}

Mc Cosker and Randall, 1977

Blackcheek moray (EN); Murène masquée (FR)

(Figure 17)

Status at Mayotte. First record from Mayotte by Wickel and Jamon (2010). Subsequently reported from Mayotte by Wickel et al. (2014) and Allaria (2016).

Observation by the author. Observed commonly. E.g.: June 2019 at Passe Bateau (12 ${ }^{\circ} 58^{\prime} 50.2^{\prime \prime}$ S$\left.44^{\circ} 58^{\prime} 58.1^{\prime \prime} \mathrm{E}\right)$ at daytime; June 2019 at 'La

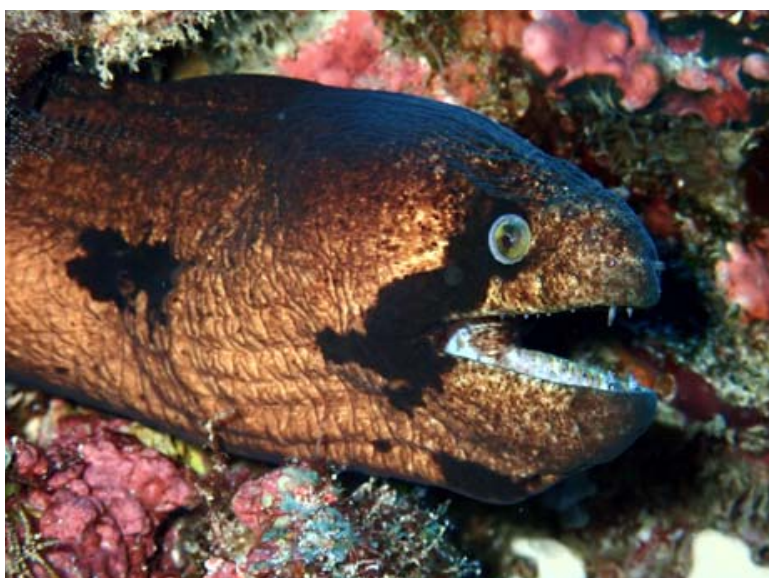

Figure 17. Gymnothorax breedeni on seaward reef, Passe Bateau (12 $58^{\prime} 50.2^{\prime \prime} \mathrm{S}-44^{\circ} 58^{\prime} 58.1^{\prime \prime} \mathrm{E}$; 30th June 2019; 10 h 14 min; 16 m depth).

cheminée' Reef barriere $\left(12^{\circ} 51^{\prime} 12.9^{\prime \prime} \mathrm{S}-45^{\circ} 17^{\prime}\right.$ $\left.21.2^{\prime \prime} \mathrm{E}\right)$ at daytime; July 2019 at Passe en S (12 $52^{\prime} 08.6^{\prime \prime} \mathrm{S}-45^{\circ} 16^{\prime} 28.8^{\prime \prime} \mathrm{E}$ ) at daytime.

Description. Small but chunky moray. Overall brown with small flecks. Black band from eyes to mouth corner and black blotch over gill opening. Maximum size $75 \mathrm{~cm}$ TL (Allen et al. 2003).

Distribution and ecology. East Africa, Seychelles, Comoros, Maldives, Line Islands to French Polynesia (Allen et al. 2003). Inhabits crevices in clear seaward reefs to depths over $25 \mathrm{~m}$ (Lieske and Myers 2005). Feed on fishes and octopuses (Bacchet et al. 2006). Tends to bite if approached.

Gymnothorax chilospilus Bleeker, 1864 Lipspot Moray (EN); Murène à lèvres tachetées (FR) (Figure 18)

Status at Mayotte. Never recorded prior to this study.

Observation by the author. Observed very commonly. E.g.: November 2016 at Sakouli beach seagrass reef flat $\left(12^{\circ} 53^{\prime} 22.2^{\prime \prime} \mathrm{S}-45^{\circ} 12^{\prime} 52.8^{\prime \prime} \mathrm{E}\right)$ at a depth of $1.5 \mathrm{~m}$ at night; March 2017 at Tsin- 


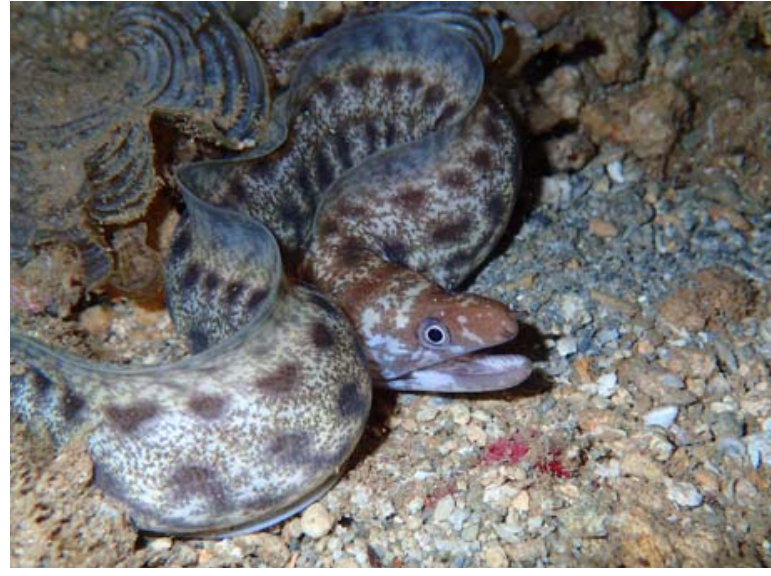

Figure 18. Gymnothorax chilospilus on reef flat, Sakouli beach $\left(12^{\circ} 53^{\prime} 22.2^{\prime \prime} \mathrm{S}-45^{\circ} 12^{\prime} 52.8^{\prime \prime} \mathrm{E}\right.$; 11 th November 2016; 19 h $13 \mathrm{~min} ; 1.5 \mathrm{~m}$ depth $\mathrm{m}$ ).

goni beach seagrass beds $\left(12^{\circ} 47^{\prime} 13.0^{\prime \prime} \mathrm{S}-45^{\circ} 05^{\prime}\right.$ $\left.38.2^{\prime \prime} \mathrm{E}\right)$ at a depth of $1 \mathrm{~m}$ at night; May 2017 at Bandrélé islet reef flat $\left(12^{\circ} 53^{\prime} 43.7^{\prime \prime} \mathrm{S}-45^{\circ} 13^{\prime}\right.$ $\left.47.8^{\prime \prime} \mathrm{E}\right)$ at a depth of $1.5 \mathrm{~m}$ at night; November 2017 at Sakouli beach seagrass beds $\left(12^{\circ} 53^{\prime}\right.$ $\left.16.7^{\prime \prime} \mathrm{S}-45^{\circ} 12^{\prime} 54.9^{\prime \prime} \mathrm{E}\right)$ at a depth of $1 \mathrm{~m}$ at night.

Description. Very small moray. Mottled reddish brown and pale yellowish with irregular dark brown blotches. Posterior part of the lower jaw has large white spots. Maximum size $52 \mathrm{~cm}$ TL (Chen et al. 1994).

Distribution and ecology. South and East Africa, Seychelles, Madagascar and Mascarenes east to Hawaiian and Society islands, north to Ryukyu Islands and Ogasawara Islands, south to Australia, Norfolk Island, and Rapa (Fricke et al. 2018). Inhabit rocky areas and shallow coral reefs from 1 to $45 \mathrm{~m}$ depth (Lieske and Myers 2005). Feed on fishes and crustaceans.

\section{Gymnothorax favagineus}

Bloch and Schneider, 1801

Honeycomb moray (EN); Murène léopard (FR)

(Figure 19)

Status at Mayotte. First record from Mayotte by Wickel and Jamon (2010). Subsequently reported from Mayotte by Wickel et al. (2014) and Allaria (2016).

Observation by the author. Observed commonly. E.g.: May 2017 at Choizil islet reef $\left(12^{\circ} 40^{\prime} 21.5^{\prime \prime} \mathrm{S}-\right.$ $45^{\circ} 03^{\prime} 03.1^{\prime \prime}$ E) at daytime; June 2017 at Passe en
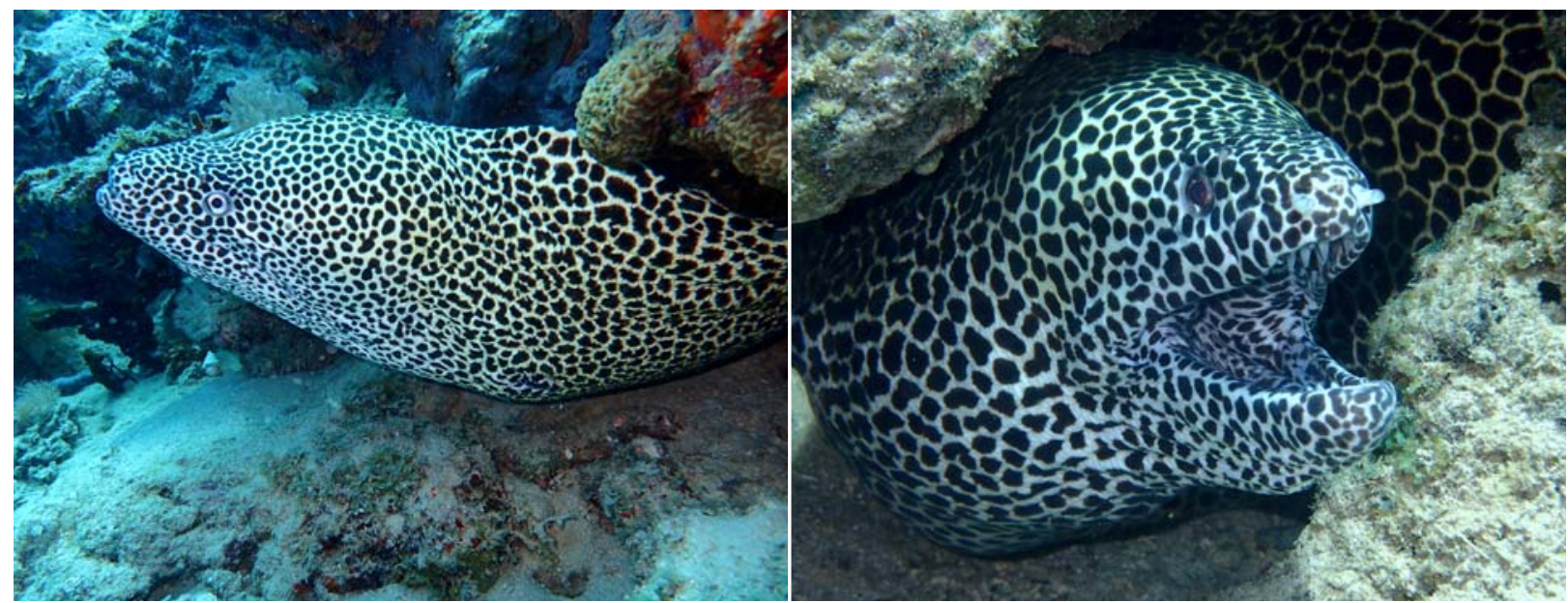

Figure 19. Left: Gymnothorax favagineus Choizil islet reef $\left(12^{\circ} 40^{\prime} 21.5^{\prime \prime} \mathrm{S}-45^{\circ} 03^{\prime} 03.1^{\prime \prime} \mathrm{E}\right.$; 4th May $2017 ; 10 \mathrm{~h} 38 \mathrm{~min} ; 5 \mathrm{~m}$ depth). Right: G. favagineus on coral reef, Passe Mtsamboro (12 37' 29.6" S-45 08' 24.6" E; 28th January 2018 ; $11 \mathrm{~h}$ $15 \mathrm{~min} ; 3 \mathrm{~m}$ depth). 
$\mathrm{S}\left(12^{\circ} 52^{\prime} 11.0^{\prime \prime} \mathrm{S}-45^{\circ} 16^{\prime} 31.1^{\prime \prime} \mathrm{E}\right)$ at daytime; January 2018 at Passe des îles Choizil $\left(12^{\circ} 42^{\prime}\right.$ 59.9" S-44 59' 19.0" E) at daytime; January 2018 at Passe Mtsamboro (12 ${ }^{\circ} 37^{\prime} 29.6^{\prime \prime} \mathrm{S}-45^{\circ}$ $08^{\prime} 24.6^{\prime \prime}$ E) at daytime.

Description. Big moray. Overall white, covered with black spots separated by narrow interspaces often forming a honeycomb-like pattern. Spots larger and more widely separated in young individuals (Smith et al. 2019). Maximum size 300 cm TL (Lieske and Myers 2005).

Distribution and ecology. Red Sea, East and South Africa, Seychelles, Madagascar and western Mascarenes east to Papua New Guinea, Japan, Australia (Fricke et al. 2018). Found on coral and rocky reefs from depths of 1-50 m. Feed on fishes and octopuses (Smith et al. 2019).

Gymnothorax fimbriatus (Bennett, 1832)

Fibriated Moray (EN); Murène frangée (FR)

(Figure 20)

Status at Mayotte. Never recorded prior to this study.

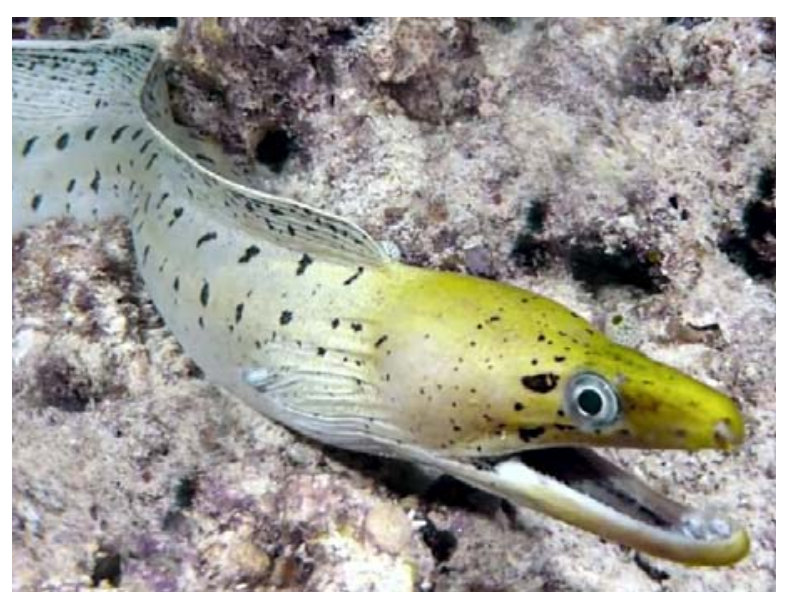

Figure 20. Gymnothorax fimbriatus at Moya beach reef flat, under dead coral $\left(12^{\circ} 47^{\prime} 16.8^{\prime \prime} \mathrm{S}-45^{\circ} 17^{\prime} 47.7^{\prime \prime} \mathrm{E}\right.$; November 2004; afternoon; depth: $4 \mathrm{~m}$ ). Reproduced with permission from Norbert Verneau.
Observation by divers. Observed very rarely. Only one time by Norbert Verneau, November 2004 on Moya beach reef flat under dead coral $\left(12^{\circ} 47^{\prime} 16.8^{\prime \prime} \mathrm{S}-45^{\circ} 17^{\prime} 47.7^{\prime \prime} \mathrm{E}\right)$ at daytime.

Description. Small moray. Yellowish head. Body with shades of brown to dirty white with highly variable pattern of widely spaced irregular dark brown spots, partial bars and bands (Allen et al. 2003). Maximum size $80 \mathrm{~cm}$ LT (Lieske and Myers 2005).

Distribution and ecology. Seychelles, Madagascar and Mascarenes east to Mariana Islands, Marquesas Islands and Tuamotu Archipelago, north to Ryukyu Islands and Ogasawara Islands, Australia and New Caledonia (Fricke et al. 2018). Found on reef flats, lagoons and external slopes up to at least $26 \mathrm{~m}$ depth. Feed on fishes and crustaceans at night (Lieske and Myers 2005).

Gymnothorax flavimarginatus (Rüppell, 1830) Yellow-edged moray (EN); Murène marbrée (FR) (Figure 21)

Status at Mayotte. First record from Mayotte by Wickel et al. (2014). Subsequently reported from Mayotte by Allaria (2016).

Observation by the author. Observed very commonly. E.g.: February 2016 near White Sand islet $\left(12^{\circ} 59^{\prime} 08.5^{\prime \prime} \mathrm{S}-45^{\circ} 12^{\prime} 53.8^{\prime \prime} \mathrm{E}\right)$ at daytime; March 2017 at Tsingoni beach reef flat $\left(12^{\circ} 47^{\prime}\right.$ 17.0" S-45 05' 37.7" E) at night; June 2017 in Bandrélé islet reef $\left(12^{\circ} 53^{\prime} 46.0^{\prime \prime} \mathrm{S}-45^{\circ} 13^{\prime} 47.2^{\prime \prime}\right.$ E) at daytime; February 2018 in Sakouli beach reef $\left(12^{\circ} 53^{\prime} 21.0^{\prime \prime} \mathrm{S}-45^{\circ} 12^{\prime} 56.0^{\prime \prime} \mathrm{E}\right)$ at daytime.

Description. Yellowish, densely and finely mottled with dark brown spots, often to the extent that little of the pale yellowish ground color remains. Yellow iris. Snout and front of lower jaw purplish brown (Smith et al. 2019). Maximum size $123 \mathrm{~cm}$ TL (Lieske and Myers 2005). 


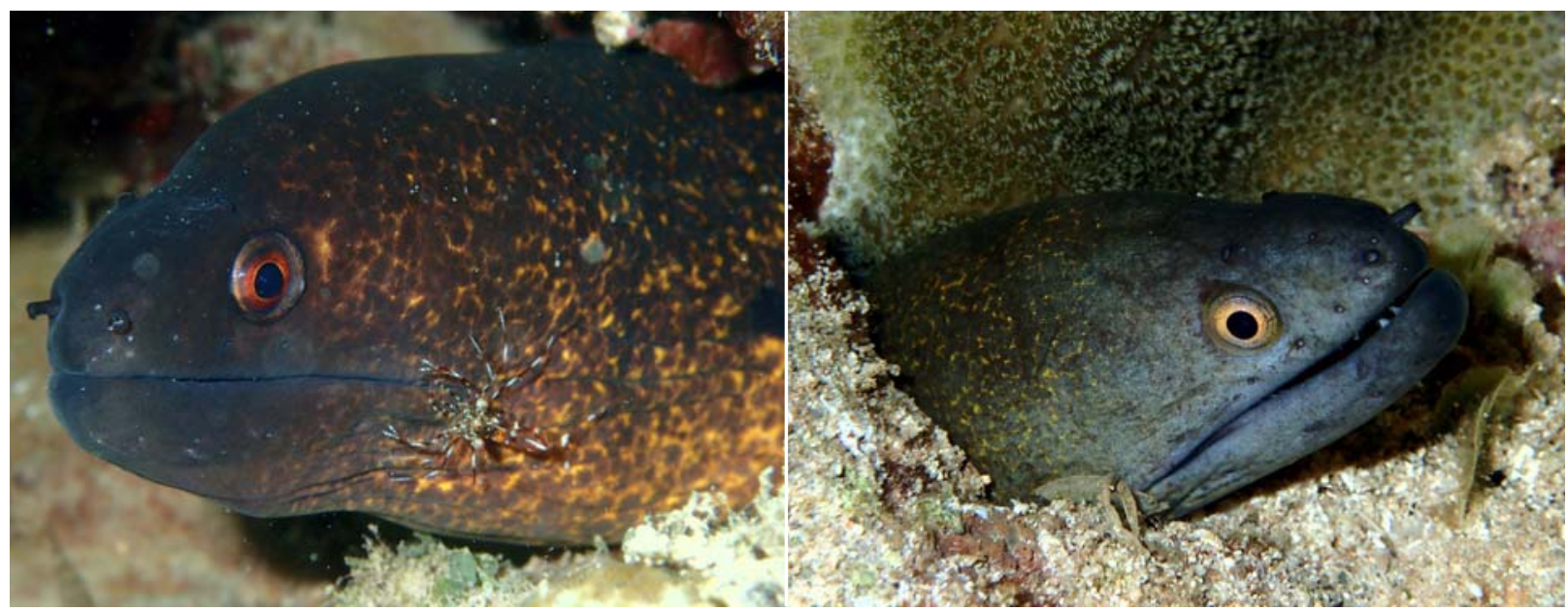

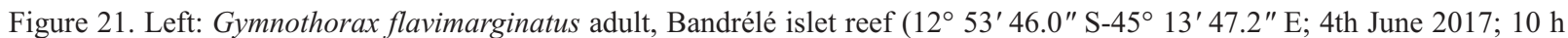
45 min; depth: $5 \mathrm{~m})$. Right: G. flavimarginatus subadult, Tsingoni beach reef flat $\left(12^{\circ} 47^{\prime} 17.0^{\prime \prime} \mathrm{S}-45^{\circ} 05^{\prime} 37.7^{\prime \prime} \mathrm{E}\right.$; 25 th March 2017; 22 h 10 min; 1 m depth).

Distribution and ecology. Red Sea, East and South Africa, Seychelles, Comoros, Madagascar and Mascarenes, east to Panama and Revillagigedo Archipelago, Ryukyu Islands, Ogasawara Islands and Hawaiian Islands, Australia, and New Caledonia (Fricke et al. 2018). Found on reef flats, lagoons and external slopes down to $150 \mathrm{~m}$. Feed on fishes and crustaceans (Lieske and Myers 2005).

Gymnothorax griseus (Lacepède, 1803) Grey moray (EN); Murène tatouée (FR)

(Figure 22)

Status at Mayotte. First record from Mayotte by Wickel et al. (2014).

Observation by the author. Observed rarely. E.g.: May 2015 at Handrema islet reef $\left(12^{\circ} 40^{\prime} 25.8^{\prime \prime} \mathrm{S}\right.$ $45^{\circ} 07^{\prime} 06.7^{\prime \prime}$ E) at daytime; March 2016 at Tsingoni beach reef $\left(12^{\circ} 47^{\prime} 13.6^{\prime \prime} \mathrm{S}-45^{\circ} 05^{\prime} 29.7^{\prime \prime} \mathrm{E}\right)$ at daytime; November 2017 at Bambo Ouest beach reef $\left(12^{\circ} 55^{\prime} 40.0^{\prime \prime} \mathrm{S}-45^{\circ} 05^{\prime} 01.9^{\prime \prime} \mathrm{E}\right)$ at daytime; April 2018 on Tsingoni beach south reef flat $\left(12^{\circ} 48^{\prime} 07.7^{\prime \prime} \mathrm{S}-45^{\circ} 05^{\prime} 54.6^{\prime \prime} \mathrm{E}\right)$ at daytime; May 2018 at Tsingoni beach reef $\left(12^{\circ} 47^{\prime} 13.6^{\prime \prime} \mathrm{S}\right.$ $45^{\circ} 05^{\prime} 29.7^{\prime \prime}$ E) at night.

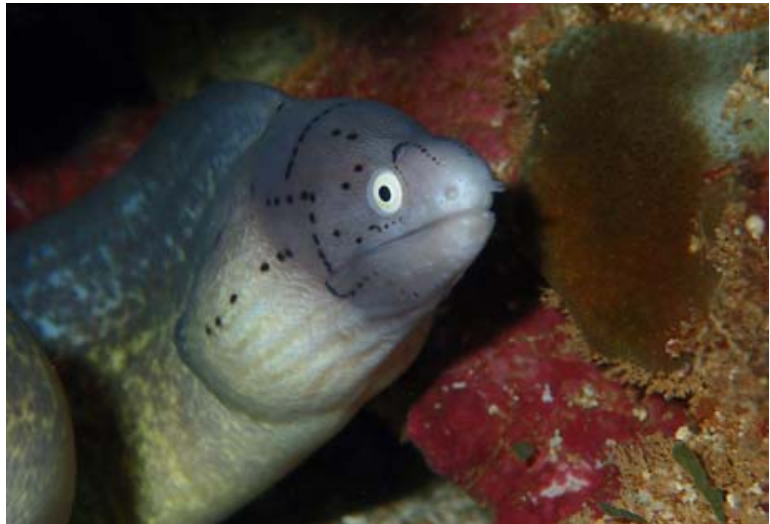

Figure 22. Gymnothorax griseus on reef flat, Tsingoni $\left(12^{\circ} 48^{\prime}\right.$ 07.7" S-45 05' 54.6" E; 21th April 2018; 15 h 39 min; $1.5 \mathrm{~m}$ depth).

Description. Small moray. Body pale yellowish or whitish gray densely mottled with irregular darker markings. Head and anterior nostril lavender-gray, the pores outlined in black. Small dark spots on head and anterior body forming conspicuous lines. Iris white. Maximum size $65 \mathrm{~cm}$ TL (Smith et al. 2019).

Distribution and ecology. Red Sea, East and South Africa, Seychelles, Madagascar and Mas- 
carenes (Fricke et al. 2018). Found in shallow water where it can be seen foraging fishes and crustaceans among corals and rocks. Reported in a depth range of 1-30 m (Smith et al. 2019).

Gymnothorax javanicus (Bleeker, 1859)

Giant Moray (EN); Murène javanaise (FR)

(Figure 23)

Status at Mayotte. First record from Mayotte by Wickel and Jamon (2010). Subsequently reported from Mayotte by Wickel et al. (2014) and Allaria (2016).

Observation by the author. Observed very commonly. The most common moray of Mayotte. E.g.: September 2015 at Handréma bay reef $\left(12^{\circ}\right.$ $\left.39^{\prime} 39.5^{\prime \prime} \mathrm{S}-45^{\circ} 06^{\prime} 22.3^{\prime \prime} \mathrm{E}\right)$ at daytime; June 2016 at Tsingoni beach reef $\left(12^{\circ} 47^{\prime} 17.8^{\prime \prime} \mathrm{S}-45^{\circ}\right.$ $\left.05^{\prime} 37.2^{\prime \prime} \mathrm{E}\right)$ at night; May 2017 on Bandrélé islet reef flat $\left(12^{\circ} 53^{\prime} 41.8^{\prime \prime} \mathrm{S}-45^{\circ} 13^{\prime} 49.3^{\prime \prime} \mathrm{E}\right)$ at night; March 2018 at White Sand islet reef $\left(12^{\circ}\right.$ $59^{\prime} 09.1^{\prime \prime} \mathrm{S}-45^{\circ} 12^{\prime} 53.8^{\prime \prime} \mathrm{E}$ ) at daytime.

Description. Biggest moray of its genus. Body and fins brown, with small, darker brown mark- ings spots (those dorsally are larger), in approximately three irregular rows. Head usually darker than the body. Gill opening in a conspicuous dark spot. Iris brown (Smith et al. 2019). Maximum size $300 \mathrm{~cm}$ TL (Lieske and Myers 2005).

Distribution and ecology. Red Sea, East Africa, Seychelles, Comoros, Madagascar and Mascarenes east to Hawaiian Islands, Pitcairn Group and Cocos Island, north to Ryukyu Islands and Ogasawara Islands, Australia and New Caledonia; Galápagos Archipelago (Fricke et al. 2018). Found on coral reefs and rocky substrates, at depths of 0$46 \mathrm{~m}$ (Smith et al. 2019). Feed on fishes and sometimes crustaceans (Lieske and Myers 2005).

Gymnothorax melatremus Schultz, 1953

Dwarf moray (EN); Murène jaunâtre (FR)

(Figure 24)

Status at Mayotte. No record prior to this study.

Observation by the author. Observed very rarely. Only one time at Mtsamboro pit, between Mtsamboro islet and White Sand islet $\left(12^{\circ} 38^{\prime} 55.9^{\prime \prime} \mathrm{S}\right.$ $\left.45^{\circ} 02^{\prime} 36.2^{\prime \prime} \mathrm{E}\right)$ at daytime in May 2018.
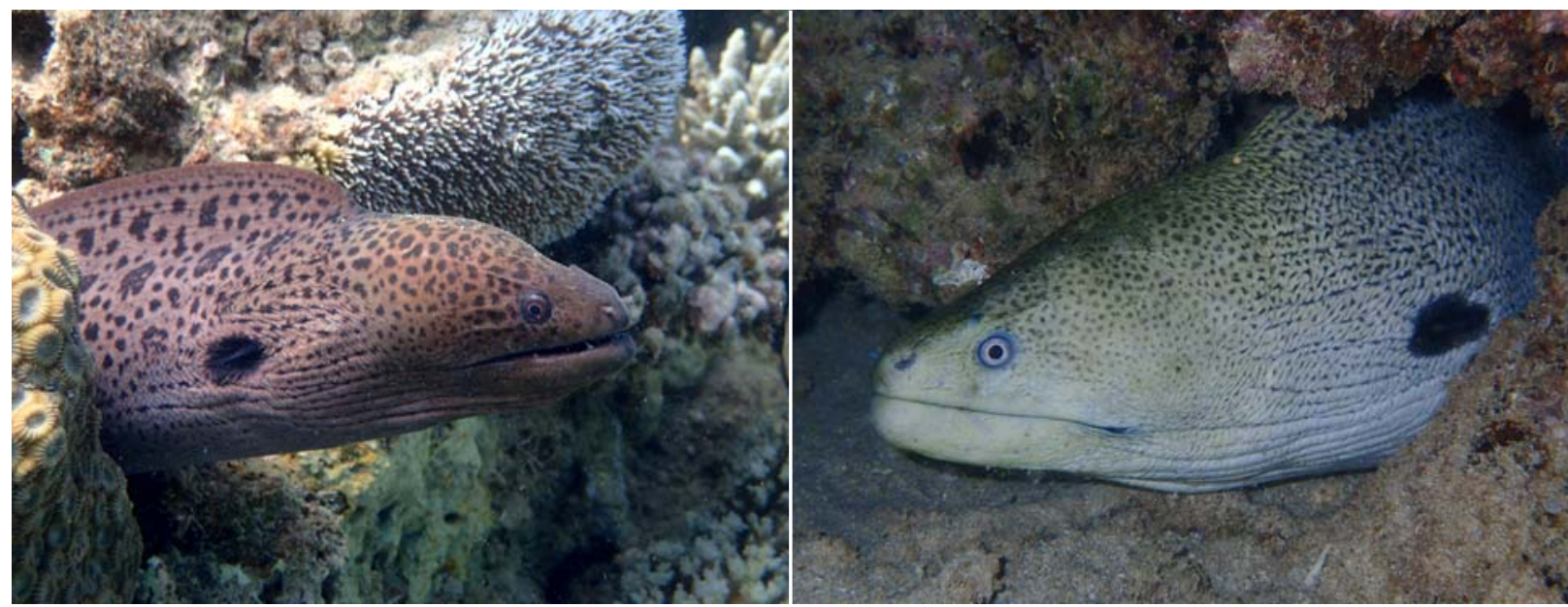

Figure 23. Left: Gymnothorax javanicus Handréma bay reef $\left(12^{\circ} 39^{\prime} 39.5^{\prime \prime}\right.$ S-450 06' 22.3" E; 15 th September 2015 ; 15 h 07 min; $2.5 \mathrm{~m}$ depth). Right: G. javanicus big adult (around $300 \mathrm{~cm} \mathrm{TL}$ ) Bandrélé islet reef flat $\left(12^{\circ} 53^{\prime} 41.8^{\prime \prime} \mathrm{S}-45^{\circ} 13^{\prime}\right.$ 49.3" E; 25th May 2017; 23 h 52 min; 1.5 m depth). 


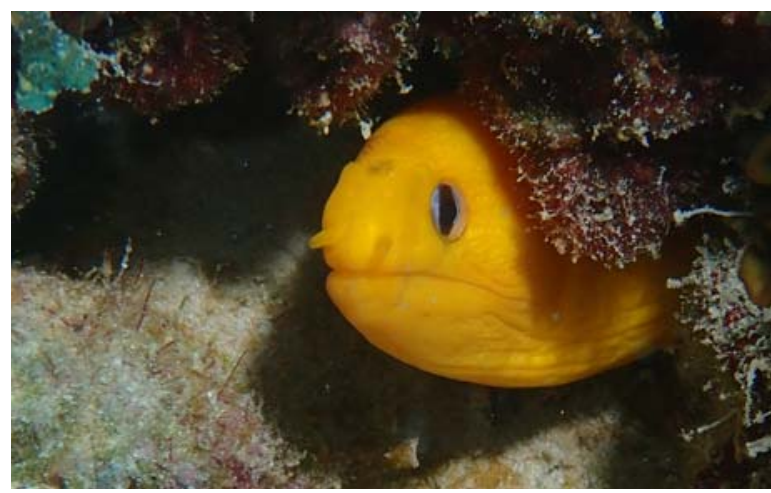

Figure 24. Gymnothorax melatremus Mtsamboro pit in coral reef $\left(12^{\circ} 38^{\prime} 55.9^{\prime \prime} \mathrm{S}-45^{\circ} 02^{\prime} 36.2^{\prime \prime} \mathrm{E}\right.$; 11 th May 2018; 14 h 2 min; 17 m depth).

Description. Very small moray. Variable brown to yellow, sometimes with dark brown markings. Bluish iris with a dark bar crossing the eye (Allen et al. 2003). Maximum size $30 \mathrm{~cm}$ TL (Allen and Erdmann 2012).

Distribution and ecology. South and East Africa, Seychelles, Madagascar and Mauritius, east to Hawaiian Islands and Pitcairn Group, Japan, Australia and New Caledonia (Fricke et al. 2018). Solitary and secretive. Found in reef crevices, primarily outer reef slopes between 1 and $58 \mathrm{~m}$ depth (Mundy 2005).

Gymnothorax meleagris (Shaw, 1795)

Turkey moray (EN); Murène ponctuée (FR) (Figure 25)

Status at Mayotte. First record from Mayotte by Wickel and Jamon (2010). Subsequently reported from Mayotte by Wickel et al. (2014) and by Allaria (2016).

Observation by the author. Observed commonly. E.g.: August 2017 at North east reef $\left(12^{\circ} 38^{\prime}\right.$ $\left.04.3^{\prime \prime} \mathrm{S}-45^{\circ} 09^{\prime} 50.6^{\prime \prime} \mathrm{E}\right)$ at daytime; January 2018 at Passe des îles Choizil (12 $42^{\circ}$ 59.9" S$44^{\circ} 59^{\prime} 19.0^{\prime \prime}$ E) at daytime; December 2018 at Sakouli beach reef $\left(12^{\circ} 53^{\prime} 20.9^{\prime \prime} \mathrm{S}-45^{\circ} 12^{\prime} 56.9^{\prime \prime}\right.$

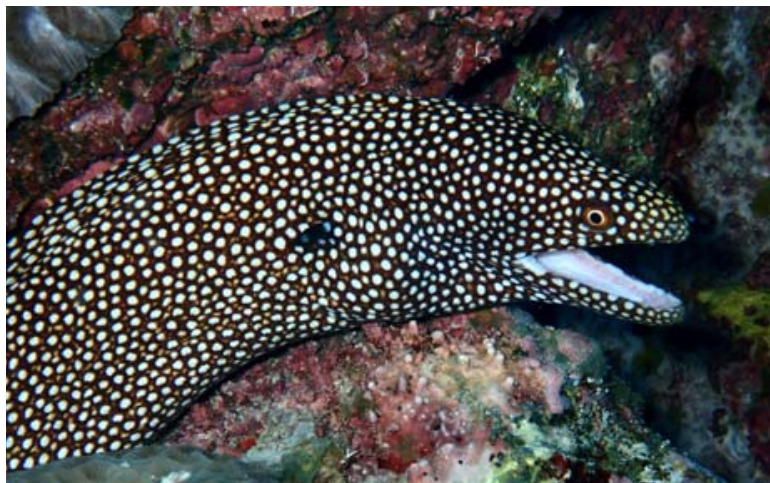

Figure 25. Gymnothorax meleagris reef, Choizil islet's Passe $\left(12^{\circ} 42^{\prime} 59.9^{\prime \prime} \mathrm{S}-44^{\circ} 59^{\prime} 19.0^{\prime \prime} \mathrm{E}\right.$; 19th January 2018; 15 h 14 min; 17 m depth).

E) at daytime; June 2019 at Passe en S $\left(12^{\circ} 52^{\prime}\right.$ $\left.17.4^{\prime \prime} \mathrm{S}-45^{\circ} 16^{\prime} 24.3^{\prime \prime} \mathrm{E}\right)$ at daytime.

Description. Inside of mouth and tip of tail white. Head and body dark brown with numerous white spots (Allen et al. 2003). Spots can be connected and form crosslinks. Maximum size $120 \mathrm{~cm}$ TL (Lieske and Myers 2005).

Distribution and ecology. East and South Africa, Seychelles, Madagascar and western Mascarenes east to Hawaiian Islands, Pitcairn Group, Galápagos Islands and Cocos Island, Japan, Ogasawara Islands and Minami Tori Shima, south to New Caledonia, Lord Howe Island and Austral Islands (Fricke et al. 2018). Found in areas rich in corals in lagoons and on the seaward reef between 1-36 $m$ depth. Feed mainly on fish and less often on crustaceans (Lieske and Myers 2005).

Gymnothorax nudivomer (Günther, 1867)

Yellowmouth moray (EN);

Murène à bouche jaune (FR) (Figure 26)

Status at Mayotte. First record from Mayotte by Allaria (2016).

Observation by divers. Observed rarely. Observed several times on the west external reef 
slopes by Norbert Verneau in November 2004 in an external east reef slopes hole during the day.

Description. Head and body light brown becoming darker posteriorly, covered with white spots, increasing in size posteriorly. Gill opening yellow surrounded with black. Inside of the mouth yellow. Eye pale with black bar (Smith et al. 2019). Maximum size $180 \mathrm{~cm}$ TL (Lieske and Myers 2005).

Distribution and ecology. Widely distributed in the Indo-Pacific from East Africa and the Red Sea to Hawaiian Islands and French Polynesia. Found

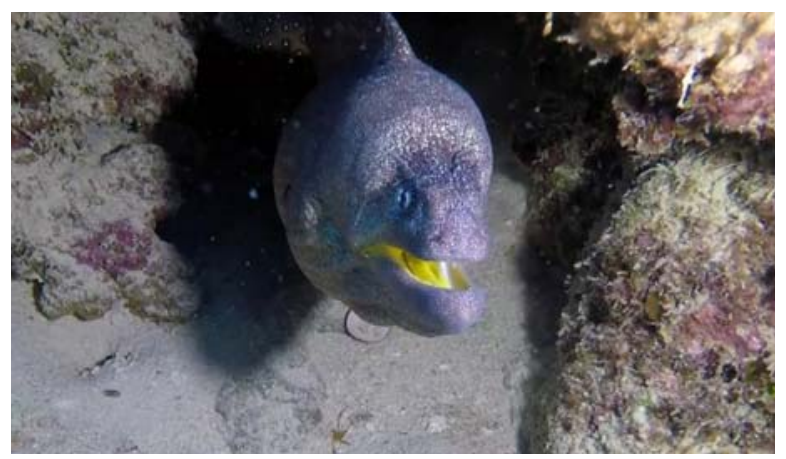

Figure 26. Gymnothorax nudivomer, external reef slopes of Moya ( $12^{\circ} 47^{\prime} 09.9^{\prime \prime} \mathrm{S}-45^{\circ} 17^{\prime} 59.5^{\prime \prime} \mathrm{E}$; November 2004, at daytime, depth: $2 \mathrm{~m}$ ). Reproduced with permission from Norbert Verneau. on seaward reefs primarily at depths of 30-271 m, occasionally shallower (Smith et al. 2019). Feed on fish at night.

Gymnothorax phasmatodes (Smith, 1962)

Ghost moray (EN); Murène fantôme (FR)

(Figure 27)

Status at Mayotte. No record prior to this study.

Observation by the author. Observed rarely. E.g.: May 2017 on Bandrélé islet sand bottom $\left(12^{\circ} 53^{\prime}\right.$ 43.0" S-45 $13^{\prime} 47.6^{\prime \prime} \mathrm{E}$ ) at night.

Observation by divers. Observed few times by Norbert Verneau. E.g.: February 2012 on Papani beach reef flat $\left(12^{\circ} 45^{\prime} 41.2^{\prime \prime} \mathrm{S}-45^{\circ} 17^{\prime} 18.8^{\prime \prime} \mathrm{E}\right)$ at night.

Description. Small moray eel. Head and body light gray, head slightly darker, head pores in pale spots. Fins with a conspicuous edge from light blue to white. Iris white. Anterior nostril pale. Maximum size $50 \mathrm{~cm}$ TL (Smith et al. 2019).

Distribution and ecology. South and East Africa, Madagascar and Mauritius, east to Philippines and Papua New Guinea, north to Taiwan, Aus-
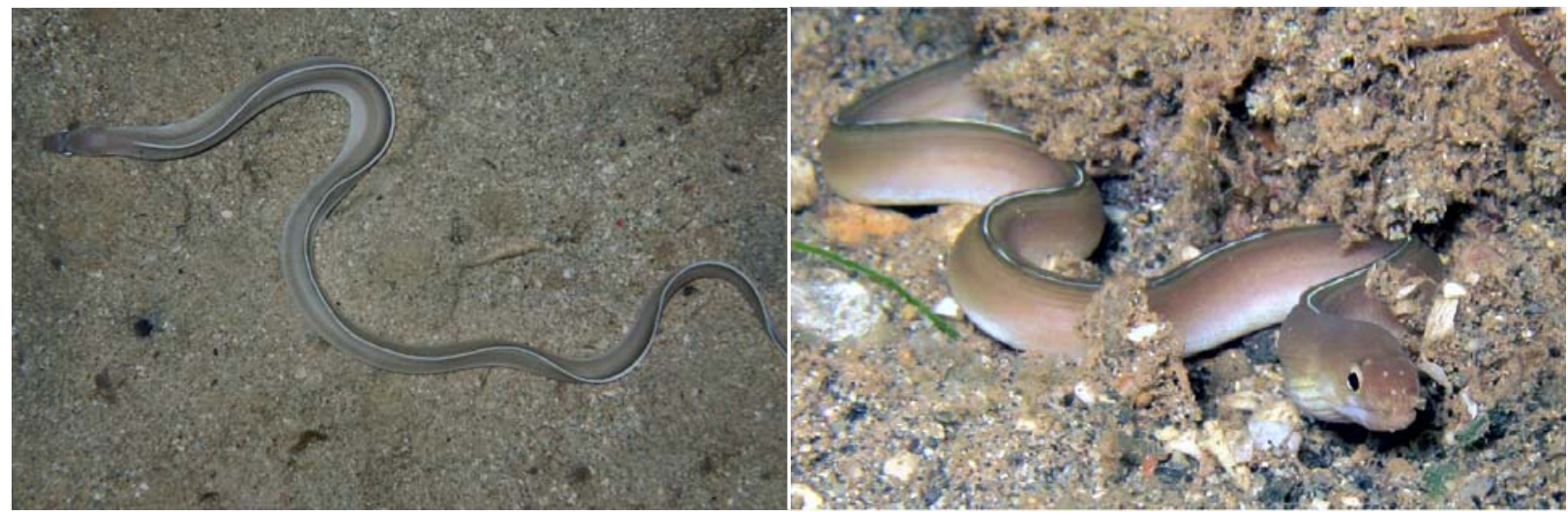

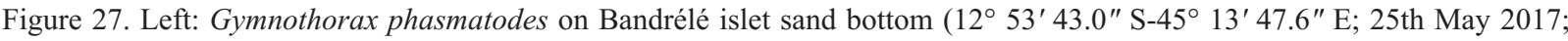
23 h 21 min; 2.5 m depth). Right: G. phasmatodes on Papani beach reef flat $\left(12^{\circ} 45^{\prime} 41.2^{\prime \prime}\right.$ S- $45^{\circ} 17^{\prime} 18.8^{\prime \prime}$ E; 17th February 2012; 19 h 46 min; depth: 2 m). Reproduced with permission from Norbert Verneau. 
tralia (Fricke et al. 2018). Found in shallow water on sand and rocky bottom from depths less than $2 \mathrm{~m}$ to $34 \mathrm{~m}$ (Smith et al. 2019). Nocturnal.

Gymnothorax pictus (Ahl, 1789)

Paintspotted moray (EN); Murène sidérale (FR) (Figure 28)

Status at Mayotte. First record from Mayotte by Wickel and Jamon (2010). Subsequently reported from Mayotte by Wickel et al. (2014) and by Allaria (2016).

Observation by the author. Observed very commonly. E.g.: October 2014 in Moya beach rock pool ( $\left.12^{\circ} 47^{\prime} 16.7^{\prime \prime} \mathrm{S}-45^{\circ} 17^{\prime} 40.8^{\prime \prime} \mathrm{E}\right)$ at daytime; March 2016 at Bandrélé islet shoreline rocks $\left(12^{\circ}\right.$ $\left.54^{\prime} 01.3^{\prime \prime} \mathrm{S}-45^{\circ} 13^{\prime} 49.3^{\prime \prime} \mathrm{E}\right)$ at daytime; May 2017 at Bandrélé islet shoreline rocks $\left(12^{\circ} 53^{\prime}\right.$ 42.6" S-45 $\left.13^{\prime} 56.3^{\prime \prime} \mathrm{E}\right)$ at daytime; May 2018 at Mtsanga Fanou shoreline rocks $\left(12^{\circ} 43^{\prime} 17.6^{\prime \prime} \mathrm{S}\right.$ $\left.45^{\circ} 02^{\prime} 26.5^{\prime \prime} \mathrm{E}\right)$ at daytime.

Description. Usually light gray, finely speckled with small dark spots, often grouped to form irregular blotches, sometimes with pale centers, sometimes with irregular edges forming a snowflake-like pattern. Iris yellowish white with irregular black circle at margin. Maximum size $120 \mathrm{~cm}$ TL (Smith et al. 2019).

Distribution and ecology. Red Sea, East and South Africa, Seychelles, Madagascar and western Mascarenes, east to Hawaiian Islands and Panama, north to Ryukyu Islands and Minami Tori Shima, Australia and Kermadec Islands (Fricke et al. 2018). Found in shallow water on reef flats and rocky shores to at least $20 \mathrm{~m}$ depth (Smith et al. 2019). Feed mainly on fish and small crustaceans (Lieske and Myers 2005).

Gymnothorax richardsonii (Bleeker, 1852)

Richardson's Moray (EN);

Murène de Richardson (FR) (Figure 29)

Status at Mayotte. No record prior to this study.

Observation by the author. Observed rarely. E.g.: May 2018 on Petite Terre airport reef flat $\left(12^{\circ} 48^{\prime}\right.$ $27.2^{\prime \prime} \mathrm{S}-45^{\circ} 16^{\prime} 34.3^{\prime \prime} \mathrm{E}$ ) at night.

Observationers. Observed few times by Norbert Verneau. For example: June 2012 on Titi Moya reef flat $\left(12^{\circ} 48^{\prime} 08.4^{\prime \prime} \mathrm{S}-45^{\circ} 17^{\prime} 10.7^{\prime \prime} \mathrm{E}\right)$ at night; June 2014 under a rock on Badamiers mudflat ( $\left.12^{\circ} 47^{\prime} 05.3^{\prime \prime} \mathrm{S}-45^{\circ} 15^{\prime} 37.4^{\prime \prime} \mathrm{E}\right)$ at daytime.
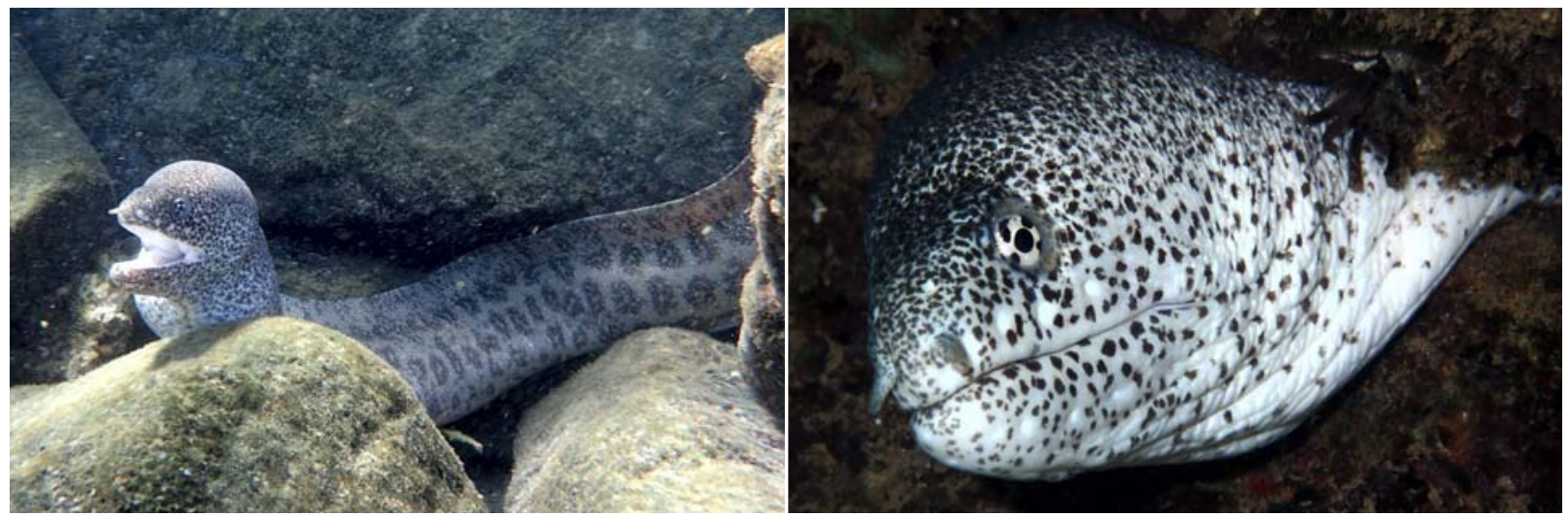

Figure 28. Left: Gymnothorax pictus Bandrélé islet south shoreline rocks (12 $54^{\prime} 01.3^{\prime \prime} \mathrm{S}-45^{\circ} 13^{\prime} 49.3^{\prime \prime} \mathrm{E}$; 4th March 2016 ; 17 h 9 min; depth: < $1 \mathrm{~m})$. Right: G. pictus Bandrélé islet North shoreline rocks $\left(12^{\circ} 53^{\prime} 42.6^{\prime \prime} \mathrm{S}-45^{\circ} 13^{\prime} 56.3^{\prime \prime} \mathrm{E}\right.$; 5th May 2017; 15 h 24 min; $1 \mathrm{~m}$ depth). 


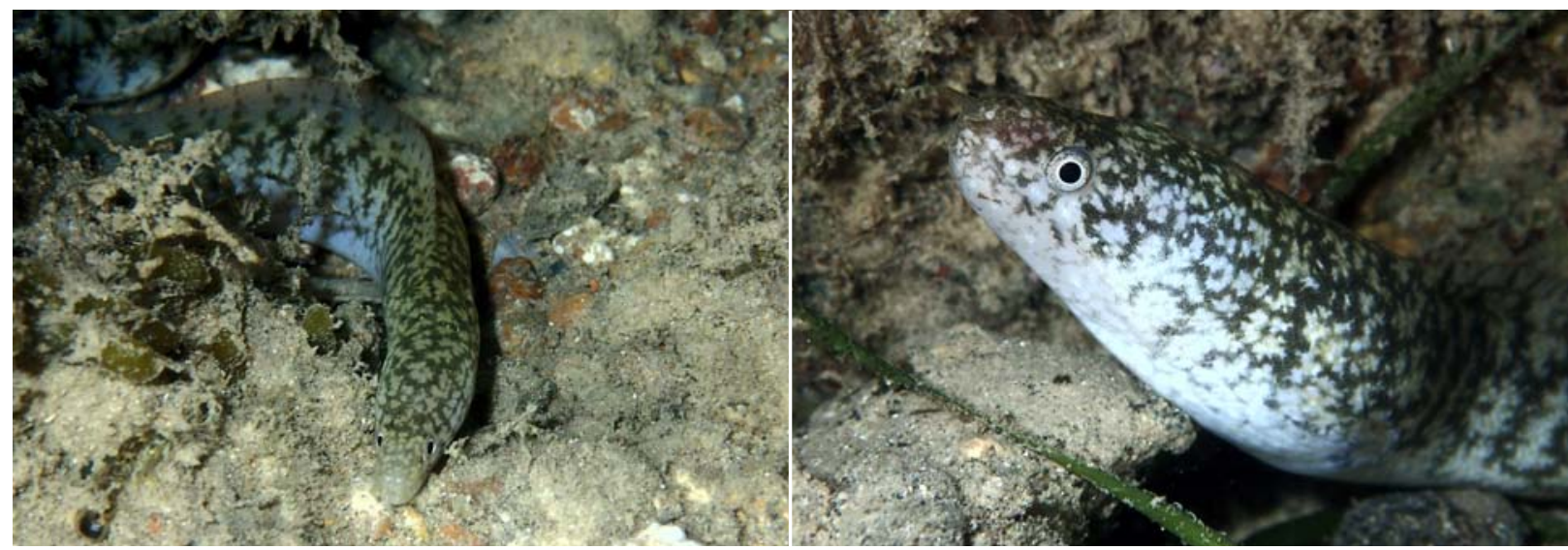

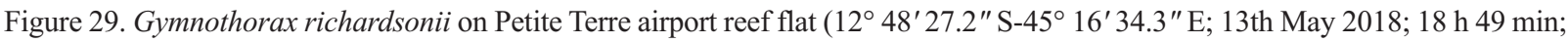
$1 \mathrm{~m}$ depth).

Description. Small moray eel. White to tan with dense brown reticulum on head, body and fins. Lighter chin and throat, and white margins around the lip pores. Maximum size $34 \mathrm{~cm}$ TL (Allen et al. 2003).

Distribution and ecology. Red Sea, East Africa, Seychelles, Madagascar and Mauritius, east to Society Islands, north to Ryukyu Islands and Ogasawara Islands, south to New Caledonia and Cook Islands (Fricke et al. 2018). Found on reef flat and estuaries between 1 and $15 \mathrm{~m}$ (Fricke et al. 2011). Nocturnal. Feed on small fishes and crustaceans (Bacchet et al. 2006).

Gymnothorax rueppelliae (McClelland, 1844) Banded moray (EN); Murène à tête jaune (FR)

(Figure 30)

Status at Mayotte. One record from Mayotte in Böhlke and Randall (2000).

Observation by the author. Observed very rarely. Only one time. May 2018 on Mtsanga Fanou reef $\left(12^{\circ} 43^{\prime} 14.7^{\prime \prime} \mathrm{S}-45^{\circ} 02^{\prime} 26.0^{\prime \prime} \mathrm{E}\right)$ at night.

Description. Light grayish brown with dark brown bars about as wide as pale interspaces. Abdomen and margin of dorsal fin between dark bars white. Top of head yellow. A dark brown spot at corner of mouth. Anterior nostrils blackish. Maximum size $80 \mathrm{~cm}$ TL (Smith et al. 2019).

Distribution and ecology. Red Sea, East Africa, Seychelles, Comoros, Madagascar and western Mascarenes, east to Hawaiian Islands, north to Ryukyu Islands, south to Western Australia and Loyalty Islands (Fricke et al. 2018). Found on coral reefs and rocky substrata at depths of 1-55 $\mathrm{m}$, often observed on reef flats at night (Smith et al. 2019). Feed on fishes and crustaceans, mainly at night. Nervous and aggressive (Lieske and Myers 2005).

Gymnothorax undulatus (Lacepède, 1803) Undulated Moray (EN); Murène ondulante (FR) (Figure 31)

Status at Mayotte. First record from Mayotte by Allaria (2016).

Observation by the author. Observed very commonly. E.g.: November 2016 at Passe en S reef $\left(12^{\circ} 52^{\prime} 09.1^{\prime \prime} \mathrm{S}-45^{\circ} 16^{\prime} 30.1^{\prime \prime} \mathrm{E}\right)$ at night; April 2017 on Sada islet reef flat $\left(12^{\circ} 50^{\prime} 51.0^{\prime \prime} \mathrm{S}-45^{\circ}\right.$ $05^{\prime} 17.1^{\prime \prime}$ E) at night; September 2017 Mtsanga 

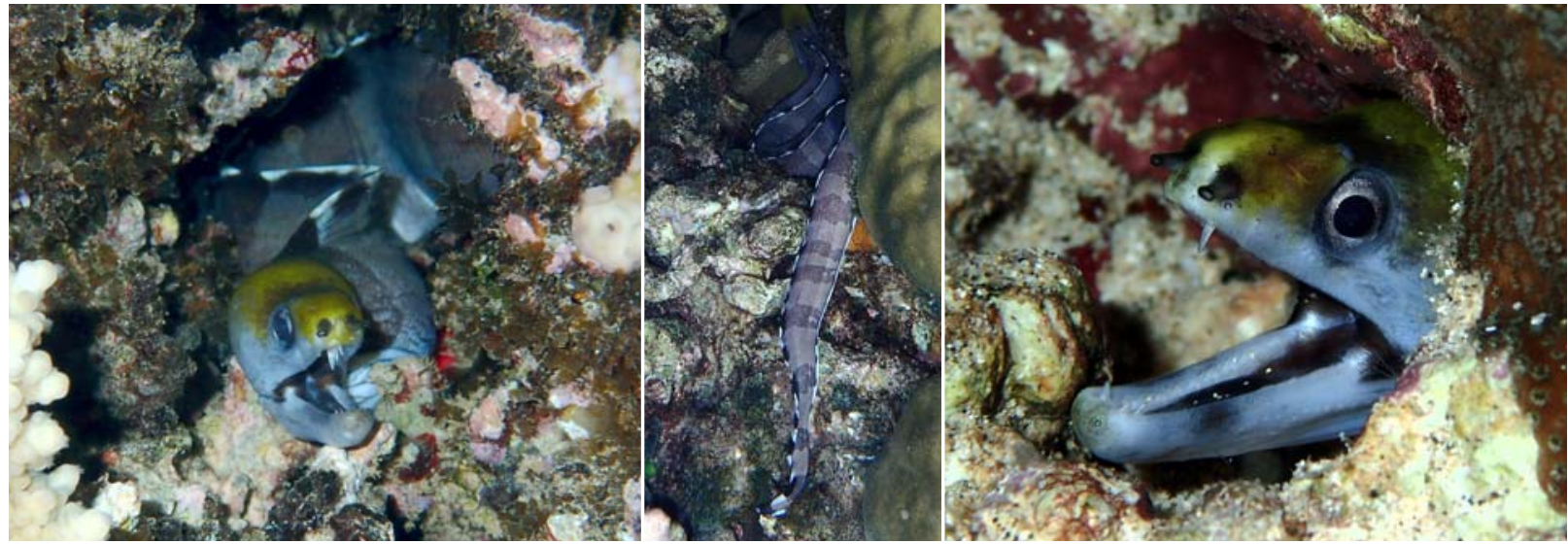

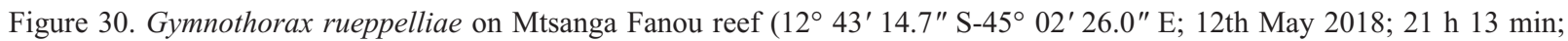
$2 \mathrm{~m}$ depth).
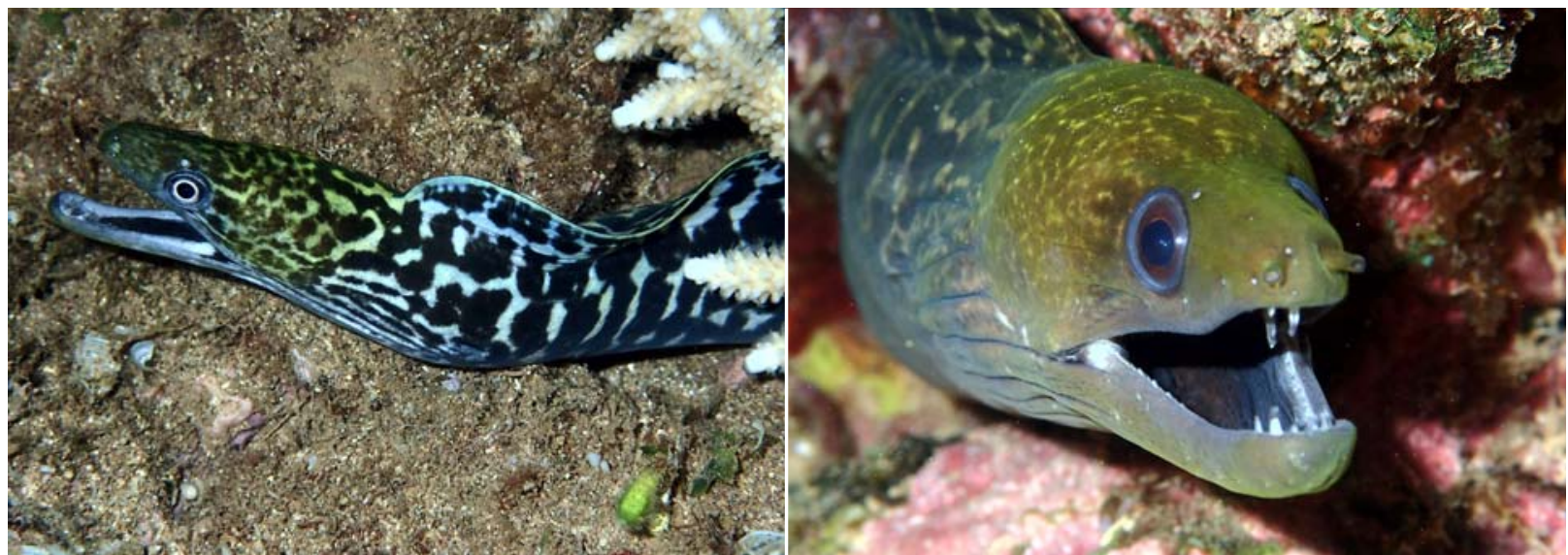

Figure 31. Left: Gymnothorax undulatus Chanfi beach reef flat $\left(12^{\circ} 45^{\prime} 21.2^{\prime \prime}\right.$ S-45 $02^{\prime} 51.9^{\prime \prime} \mathrm{E} ; 27$ th February $2018 ; 22 \mathrm{~h}$ 19 min; depth: $1.5 \mathrm{~m})$. Right: G. undulatus on Mtsanga Fanou reef at daytime $\left(12^{\circ} 43^{\prime} 21.2^{\prime \prime}\right.$ S- $45^{\circ} 02^{\prime} 18.6^{\prime \prime} \mathrm{E} ; 24$ th September 2017; 14 h 35 min; 3 m depth).

Fanou reef $\left(12^{\circ} 43^{\prime} 21.2^{\prime \prime} \mathrm{S}-45^{\circ} 02^{\prime} 18.6^{\prime \prime} \mathrm{E}\right)$ at daytime; February 2018 on Chanfi beach reef flat $\left(12^{\circ} 45^{\prime} 21.2^{\prime \prime} \mathrm{S}-45^{\circ} 02^{\prime} 51.9^{\prime \prime} \mathrm{E}\right)$ at night; December 2018 at Ngouja reef $\left(12^{\circ} 57^{\prime} 50.1^{\prime \prime}\right.$ S$\left.45^{\circ} 05^{\prime} 02.0^{\prime \prime} \mathrm{E}\right)$ at night.

Description. Variable color across range. Dark brown to black, with narrow pale markings usually interconnected to form a reticulation. The pattern generally more obscure in larger specimens. Top of head yellow-green, densely spotted with small irregular dark brown spots. Snout and lower jaw yellow-green to light grey-brown without spots. Corner of mouth with a white spot. Maximum size $150 \mathrm{~cm}$ TL (Smith et al. 2019).

Distribution and ecology. Red Sea, East and South Africa, Seychelles, Comoros, Madagascar and Mascarenes, east to Panama and Revillagigedo Archipelago, Japan, Ogasawara, Hawaiian and Gambier islands, Australia and New Caledonia (Fricke et al. 2018). Found in shallow water on 
coral reef and seaward reef up to $110 \mathrm{~m}$ depth (Mundy 2005). Common on reef flats. Feed on fish, octopus and probably crustaceans at night (Lieske and Myers 2005).

Gymnothorax zonipectis Seale, 1906 Barredfin Moray (EN);

Murène à nageoire rayée (FR) (Figure 32)

Status at Mayotte. First record from Mayotte by Allaria (2016).

Observation by the author. Observed rarely. E.g.: November 2016 at Passe en $\mathrm{S}$ reef $\left(12^{\circ} 52^{\prime}\right.$ 09.1" S-45 $16^{\prime} 30.1^{\prime \prime}$ E) at night; April 2018 at Passe en S reef $\left(12^{\circ} 52^{\prime} 03.1^{\prime \prime} \mathrm{S}-45^{\circ} 16^{\prime} 21.3^{\prime \prime} \mathrm{E}\right)$ at night; December 2018 at Passe en S reef $\left(12^{\circ}\right.$ $\left.52^{\prime} 06.1^{\prime \prime} \mathrm{S}-45^{\circ} 16^{\prime} 25.4^{\prime \prime} \mathrm{E}\right)$ at night.

Description. Small moray. Tan with longitudinal rows of brown blotches on body and bands on fins. Characteristic designs on the head with white bands and blotches on jaws and a dark brown band at rear edge of eye (Allen et al. 2003). Maximum size $47 \mathrm{~cm}$ TL (Lieske and Myers 2005).

Distribution and ecology. South and East Africa, Seychelles, Madagascar and western Mascarenes, east to Society Islands and Marquesas Islands, north to Taiwan and southern Japan, Australia and New Caledonia (Fricke et al. 2018). Found in reef crevices, mainly of seaward reef in 4 to $40 \mathrm{~m}$ depth (Allen et al. 2003). Feed on small fishes and crustaceans at night (Bacchet et al. 2006).

Pseudechidna brummeri (Bleeker, 1858)

White ribbon eel (EN);

Murène à ruban blanc (FR) (Figure 33)

\section{Status at Mayotte}

First record from Mayotte by Wickel and Jamon (2010). Subsequently reported from Mayotte by Wickel et al. (2014) and Fricke et al. (2018).
Observation by the author. Observed rarely. E.g.: October 2014 at Bandrélé reef on reef flat $\left(12^{\circ}\right.$ $\left.54^{\prime} 33.1^{\prime \prime} \mathrm{S}-45^{\circ} 14^{\prime} 19.3^{\prime \prime} \mathrm{E}\right)$ at daytime; July 2016 on N'gouja reef flat $\left(12^{\circ} 57^{\prime} 45.6^{\prime \prime} \mathrm{S}-45^{\circ} 04^{\prime}\right.$ $\left.59.6^{\prime \prime} \mathrm{E}\right)$ at daytime.

Observation by divers. Observed few times by Norbert Verneau. E.g.: on Titi Moya reef flat $\left(12^{\circ}\right.$ 48' 08.2" S-45 17' 22.2" E); October 2011 on Papani reef flat $\left(12^{\circ} 45^{\prime} 34.7^{\prime \prime} \mathrm{S}-45^{\circ} 17^{\prime} 23.4^{\prime \prime} \mathrm{E}\right)$ at daytime.

Description. Long laterally compressed body. Pale brown to pale green with small dark spots on head. Narrow light blue to white margin on fins. Maximum size $103 \mathrm{~cm}$ TL (Allen et al. 2003).

Distribution and ecology. East Africa, Mayotte and Madagascar east to Mariana Islands and Samoa, north to southern Japan, south to New Caledonia (Fricke et al. 2018). Found on shallow bottoms on reef flats and lagoons, usually buried in sand or scree areas (Lieske and Myers 2005). Between 1 to $8 \mathrm{~m}$ depth (Allen et al. 2003). Feed mainly on fish and small crustaceans (Lieske and Myers 2005).

Rhinomuraena quaesita Garman, 1888

Ribbon Moray (EN); Murène ruban (FR) (Figure 34)

Status at Mayotte. First record from Mayotte by Wickel and Jamon (2010). Subsequently reported from Mayotte by Wickel et al. (2014) and Allaria (2016).

Observation by the author. Observed rarely. Observed several times always in the same area. E.g.: June 2017 Bandrélé islet on reef flat $\left(12^{\circ} 53^{\prime}\right.$ 41.0" S-45 $13^{\prime}$ 51.6" E) at daytime; December 2018 Bandrélé islet on reef flat $\left(12^{\circ} 53^{\prime} 41.0^{\prime \prime} \mathrm{S}\right.$ $\left.45^{\circ} 13^{\prime} 51.6^{\prime \prime} \mathrm{E}\right)$ at daytime; June 2019 Passe en $\mathrm{S}\left(12^{\circ} 52^{\prime} 17.4^{\prime \prime} \mathrm{S}-45^{\circ} 16^{\prime} 26.2^{\prime \prime} \mathrm{E}\right) 12 \mathrm{~m}$ depth at daytime; July 2019 Passe en S (12 52' 06.1" S$\left.45^{\circ} 16^{\prime} 25.7^{\prime \prime} \mathrm{E}\right)$ at $16 \mathrm{~m}$ depth at daytime. 

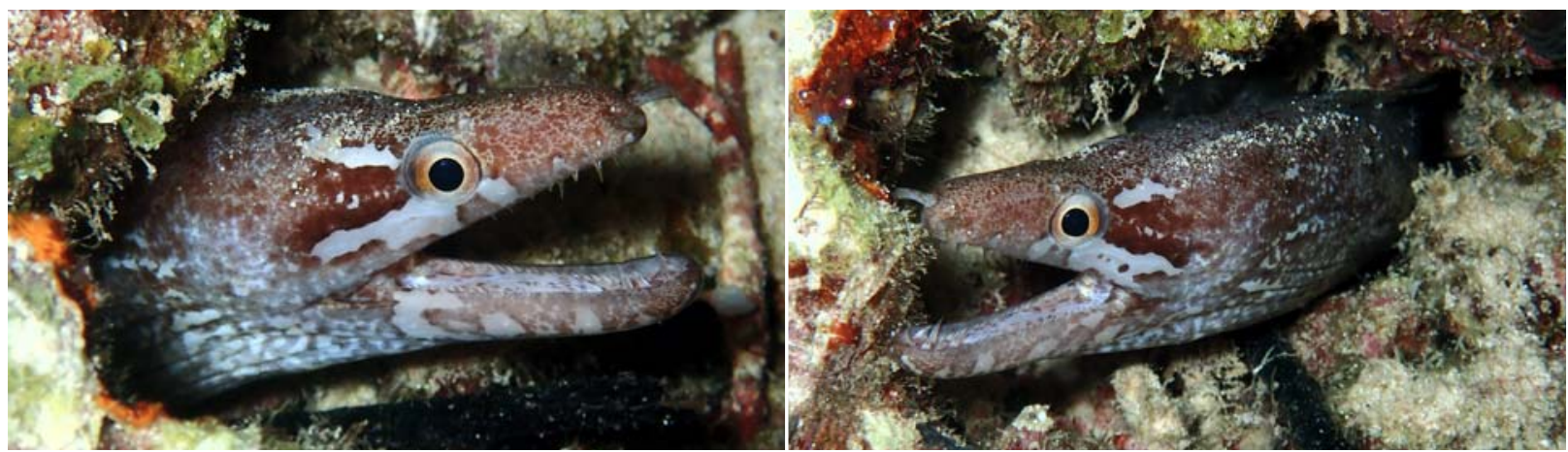

Figure 32. Gymnothorax zonipectis at Passe en S reef (12 52'03.1" S-45 16'21.3" E; 12th April 2018; 19 h 17 min; 13 m depth).

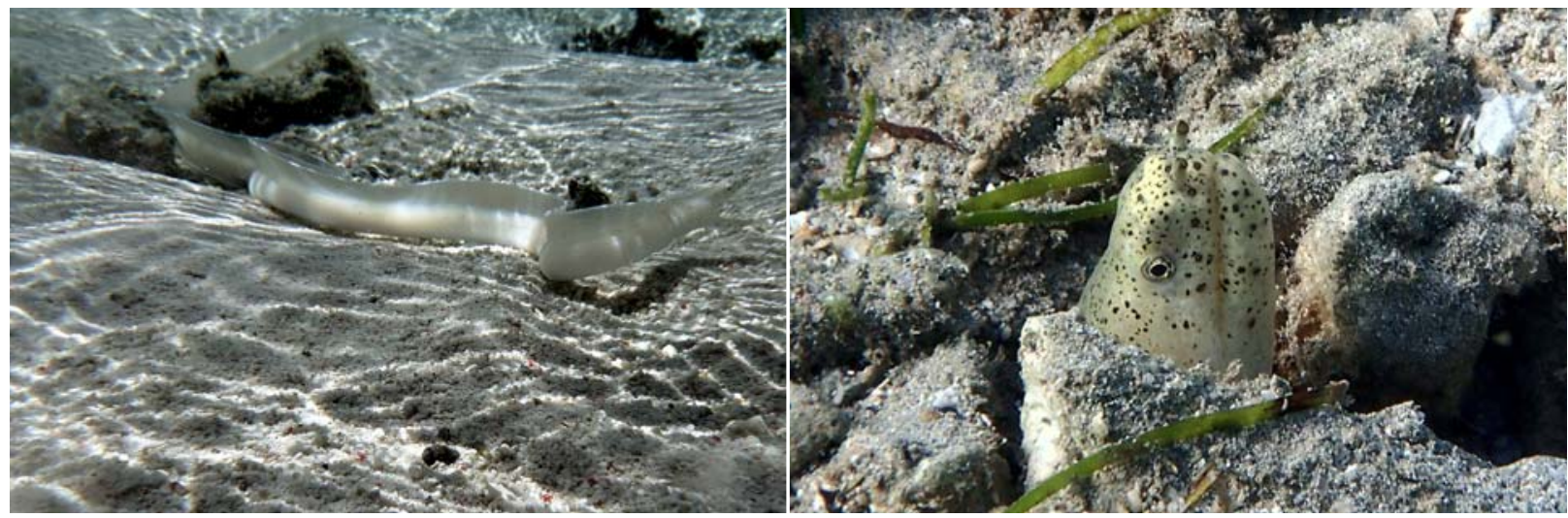

Figure 33. Left: Pseudechidna brummeri at Bandrélé reef on reef flat $\left(12^{\circ} 54^{\prime} 33.1^{\prime \prime} \mathrm{S}-45^{\circ} 14^{\prime} 19.3^{\prime \prime} \mathrm{E}\right.$; 8th October 2014 ; 9 h $13 \mathrm{~min} ;<1 \mathrm{~m}$ depth). Right: P. brummeri on N'gouja reef flat $\left(12^{\circ} 57^{\prime} 45.6^{\prime \prime} \mathrm{S}-45^{\circ} 04^{\prime} 59.6^{\prime \prime} \mathrm{E} ; 16\right.$ th July 2016 ; $17 \mathrm{~h}$ $25 \mathrm{~min} ; 3 \mathrm{~m}$ depth).

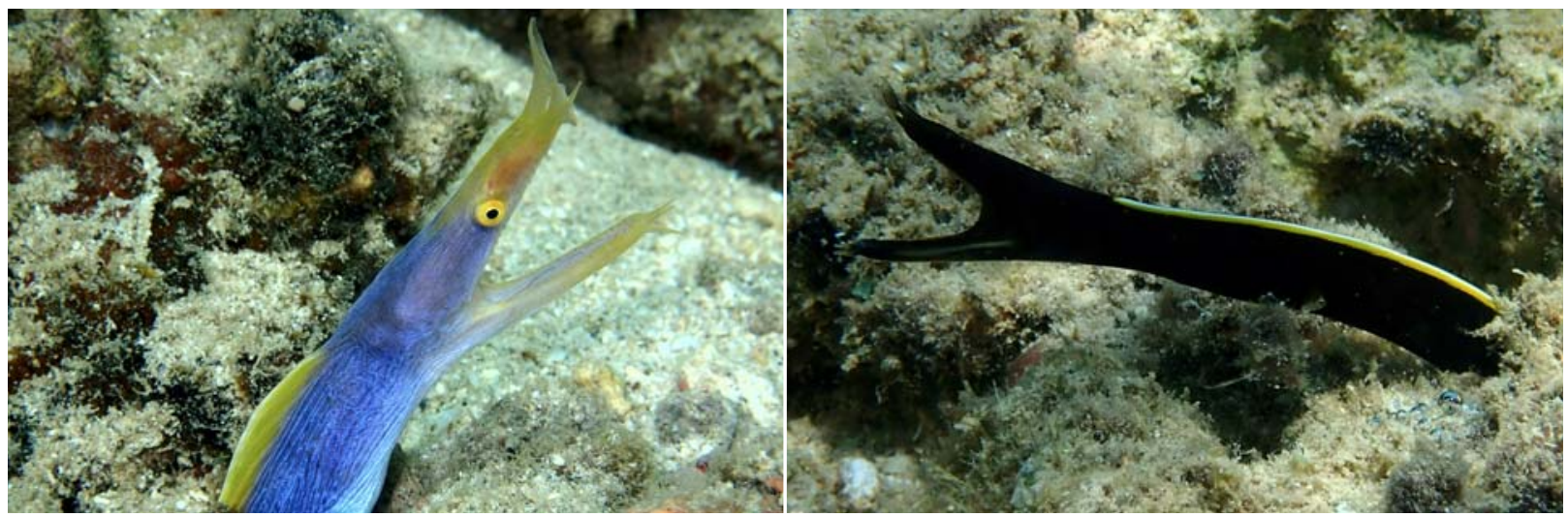

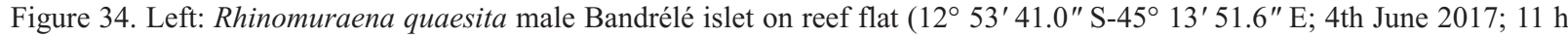
$38 \mathrm{~min} ; 2.5 \mathrm{~m}$ depth). Right: $R$. quaesita juvenile Bandrélé islet on reef flat $\left(12^{\circ} 53^{\prime} 41.0^{\prime \prime} \mathrm{S}-45^{\circ} 13^{\prime} 51.6^{\prime \prime} \mathrm{E}\right.$; 9th December 2018; 12 h 59 min; 2 m depth). 
Description. Long laterally compressed body. Have three fleshy tentacles on the tip of its lower jaw, a single fleshy pointed projection at the tip of its snout, and tubular anterior nostrils ending in fanlike expansions. Juveniles are completly black with yellow on the dorsal fin and a little on the lower jaw. Female are nearly all yellow. Males are brillant blue with yellow dorsal fin, snout, lower jaw, gill opening and iris. Maximum size $120 \mathrm{~cm}$ TL (Lieske and Myers 2005).

Distribution and ecology. East Africa, Seychelles, Madagascar and western Mascarenes, east to Mariana Islands and Tuamotu Archipelago, Japan and Ogasawara Islands, Australia and New Caledonia (Fricke et al. 2018). Found between 1 to $67 \mathrm{~m}$ depth (Allen and Erdmann 2012) in the lagoon and seaward reefs. A secretive species normally hidden in sand or rubble, sometimes with only its head protruding. Feed mainly on fish (Lieske and Myers 2005).

Scuticaria tigrina (Lesson, 1828)

Tiger reef-eel (EN); Murène tigre (FR)

(Figure 35)

Status at Mayotte. First record from Mayotte by Allaria (2016).

Observation by the author. Observed very rarely. One time in August 2015 on Musicale beach reef $\left(12^{\circ} 55^{\prime} 13.2^{\prime \prime} \mathrm{S}-45^{\circ} 11^{\prime} 10.4^{\prime \prime} \mathrm{E}\right)$ by the end of the day in $1.5 \mathrm{~m}$ depth.

Observation by divers. Two observations by Norbert Verneau in December 2004 at Badamiers reef $\left(12^{\circ} 45^{\prime} 26.1^{\prime \prime} \mathrm{S}-45^{\circ} 16^{\prime} 55.0^{\prime \prime} \mathrm{E}\right)$ at night.

Description. Nearly cylindrical body with rounded snout and jaw. Head posterior to corner of mouth and body pale yellowish to light brown, with well-separated, irregularly round, dark brown spots of variable size. Head anterior to the corner of the mouth with many small dark brown

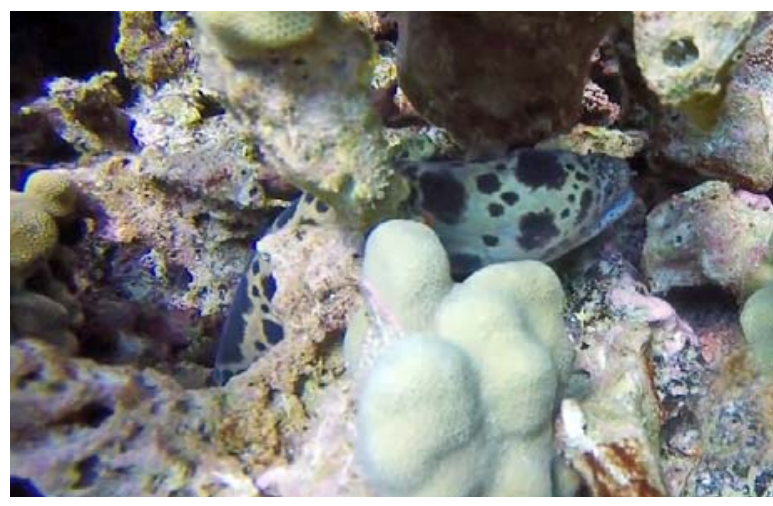

Figure 35. Scuticaria tigrina at Badamiers reef $\left(12^{\circ} 45^{\prime}\right.$ 26.1" S-45 ${ }^{\circ} 16^{\prime} 55.0^{\prime \prime} \mathrm{E}$; December 2004; $1 \mathrm{~m}$ depth). Reproduced with permission from Norbert Verneau.

spots. Maximum size about $140 \mathrm{~cm}$ TL (Smith et al. 2019).

Distribution and ecology. East Africa, South Africa, Seychelles, Madagascar and western Mascarenes, east to Panama and Revillagigedo Archipelago, north to Taiwan, Kerama and Hawaiian islands, New Caledonia (Fricke et al. 2018). Found in lagoon and seaward reefs between rocks or coral. Extremely secretive (Lieske and Myers 2005). Maximum reported depth of 25 m (Allen and Erdmann 2012). Feed at night on other moray eel. Opportunistic scavenger observed feeding on the remains of a goat.

Strophidon sathete (Hamilton, 1822)

Giant slender moray (EN);

Murène fil géante (FR) (Figure 36)

Status at Mayotte. First record by Deliot (2000). Subsequently reported from Mayotte by Wickel and Jamon (2010) and Wickel et al. (2014).

Observation by the author. No observation. Observed very rarely.

Description. Body very elongate. Snout long, jaws of equal length. Eye small, close to the tip of 


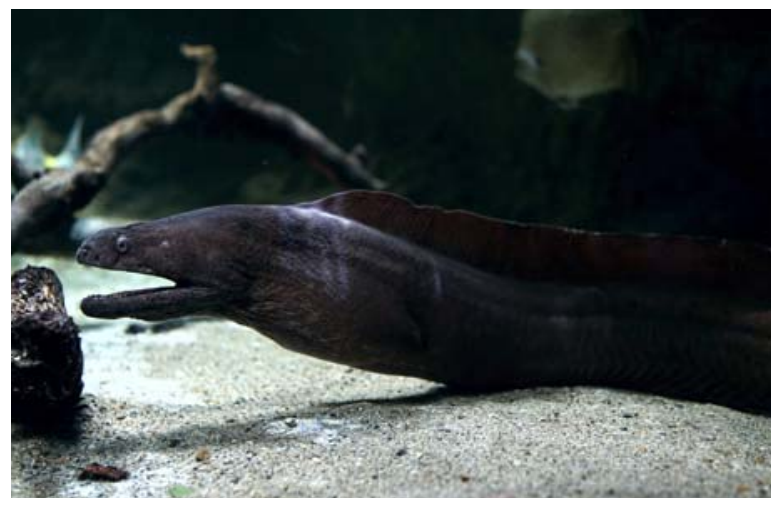

Figure 36. Strophidon sathete. Reproduced with permission from the Okinawa Commemorative National Government Park (Ocean Expo Park), Okinawa Churaumi Aquarium.

snout. Anterior nostril tubular; posterior above and slightly behind anterior margin of the eye. Grayish brown, paler ventrally, fins darker (Smith et al. 2019). Maximum size $394 \mathrm{~cm}$ TL (Lieske and Myers 2005).

Distribution and ecology. Red Sea, East and South Africa, Madagascar and western Mascarenes, east to Mariana Islands and Society Islands, Japan, Australia and New Caledonia (Fricke et al. 2018). Found on muddy bottoms near river mouths and bays between 0 to $15 \mathrm{~m}$ depth. Have been observed emerging vertically from a burrow (Lieske and Myers 2005). Feed mainly on a variety of small fishes and crustaceans.

Uropterygius micropterus (Bleeker, 1852)

Tidepool snake moray $(\mathrm{EN})$;

Murène-serpent des mares (FR) (Figure 37)

Status at Mayotte. Never recorded prior to this study.

Observation by divers. Observed very rarely. Observed few times by Norbert Verneau. E.g.: June 2013 on Titi Moya flat reef $\left(12^{\circ} 48^{\prime} 08.4^{\prime \prime}\right.$ S$\left.45^{\circ} 17^{\prime} 10.7^{\prime \prime} \mathrm{E}\right)$ at night.

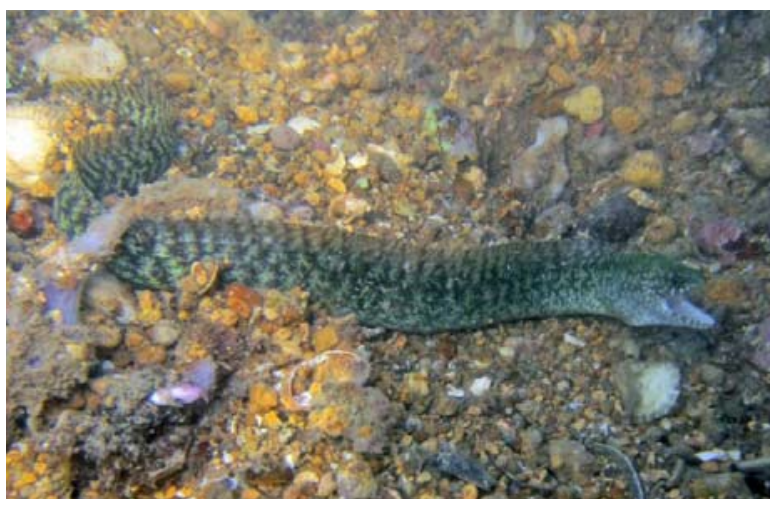

Figure 37. Uropterygius micropterus on Titi Moya reef flat $\left(12^{\circ} 48^{\prime} 08.4^{\prime \prime} \mathrm{S}-45^{\circ} 17^{\prime} 10.7^{\prime \prime} \mathrm{E} ; 25\right.$ th June 2013; $22 \mathrm{~h} 12 \mathrm{~min}$; depth: $1 \mathrm{~m}$ ). Reproduced with permission from Norbert Verneau.

Description. Small moray eel. Light brown with irregular dark brown lines on upper two-thirds of head and body, partly interconnected to form a fine reticulum. Dorsal fin reduced. Maximum size about $25 \mathrm{~cm}$ TL (Smith et al. 2019).

Distribution and ecology. Red Sea, East Africa and Madagascar, east to Phoenix Islands, north to Ryukyu Islands (Fricke et al. 2018). Found on rubble areas of intertidal reef flats, tide pools, and shallow reefs to a depth of $10 \mathrm{~m}$ (Smith et al. 2019). Feed on small crustaceans and probably on small fishes (Allen and Erdmann 2012).

Uropterygius nagoensis Hatooka, 1984

Nago snakemoray (EN);

Murène-serpent de Nago (FR) (Figure 38)

Status at Mayotte. Never recorded prior to this study.

Observation by the author. Observed very rarely. One time in May 2017 Bandrélé islet reef $\left(12^{\circ} 53^{\prime}\right.$ $39.3^{\prime \prime} \mathrm{S}-45^{\circ} 13^{\prime} 48.7^{\prime \prime} \mathrm{E}$ ) at night.

Description. Snout moderately long. Curved jaws with many dagger-like teeth. Eye closer to the tip of snout than to the rictus. Anterior nostril tubu- 


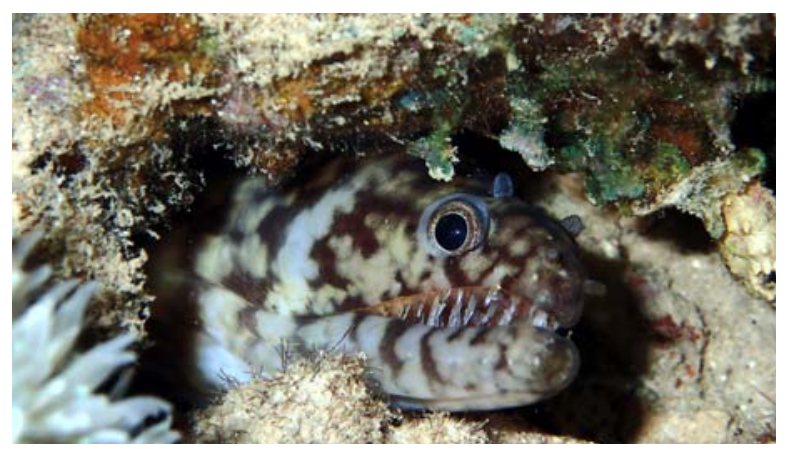

Figure 38. Uropterygius nagoensis at Bandrélé islet reef $\left(12^{\circ} 53^{\prime}\right.$ 39.3" S-45 13' 48.7" E; 25th May 2017; 21 h 21 min; $1.5 \mathrm{~m}$ depth).

lar; posterior nostril protruding above the anterior margin of the eye. Tan with large vertically aligned dark brown dendritic markings interconnected to form a thick reticular pattern. An irregular white band across interorbital space. Maximum size about $80 \mathrm{~cm}$ TL (Smith et al. 2019).

Distribution and ecology. Red sea, western and central Pacific, Society and Solomon Islands, Japan, Taiwan, Papua New Guinea and Australia (Smith et al. 2019). Secretive, found in caves and crevices of fringing reefs at depths of 3 to $30 \mathrm{~m}$ depth (Allen and Erdmann 2012; Smith et al. 2019). Tooth shape of this species indicates a piscivore diet.

Uropterygius polyspilus (Regan, 1909)

Large-spotted snake moray (EN);

Murène-serpent tachetée (FR) (Figure 39)

Status at Mayotte. One record from Mayotte in Böhlke and Randall (2000).

Observation by the author. Observed rarely. E.g.: June 2016 Tsingoni beach reef $\left(12^{\circ} 47^{\prime} 16.2^{\prime \prime} \mathrm{S}-45^{\circ}\right.$ 05' 31.7" E) at night; December 2016 Sakouli beach reef $\left(12^{\circ} 53^{\prime} 18.0^{\prime \prime} \mathrm{S}-45^{\circ} 12^{\prime} 58.7^{\prime \prime} \mathrm{E}\right)$ at night; May 2018 Fanou beach reef $\left(12^{\circ} 43^{\prime} 13.6^{\prime \prime} \mathrm{S}-\right.$ $\left.45^{\circ} 02^{\prime} 23.6^{\prime \prime} \mathrm{E}\right)$ at night; May 2018 Tsingoni beach reef $\left(12^{\circ} 47^{\prime} 16.7^{\prime \prime} \mathrm{S}-45^{\circ} 05^{\prime} 34.8^{\prime \prime} \mathrm{E}\right)$ at night.
Description. Small moray. Tan to white, with rounded dark brown spots. Spots on head smaller than those on body. Nostrils white. Sometimes with a white area on the snout between the nostrils. Maximum size at least $78 \mathrm{~cm}$ TL (Smith et al. 2019).

Distribution and ecology. Red Sea, Zanzibar, Comoro Islands, Seychelles, Chagos Archipelago, Australia, Vietnam, Philippines, Caroline Islands, Hawaiian Islands, Johnston Island, Samoa Islands, Line Islands, and Society Islands. Found on reef flats and coral reefs at depth of 18 $m$ depth (Smith et al. 2019). Feed on shrimps and fishes (Bacchet et al. 2006).

Uroptegygius sp. (undescribed)

(Figure 40)

Status at Mayotte. No record prior to this study.

Observation by the author. Observed very rarely. One time in February 2018 at Chanfi beach reef $\left(12^{\circ} 45^{\prime} 19.3^{\prime \prime} \mathrm{S}-45^{\circ} 03^{\prime} 32.8^{\prime \prime} \mathrm{E}\right)$ at night.

Observation by divers. Observed by Nicolas Bouteiller in January 2020 Sakouli beach reef flat $\left(12^{\circ} 53^{\prime} 15.9^{\prime \prime} \mathrm{S}-45^{\circ} 13^{\prime} 00.2^{\prime \prime} \mathrm{E}\right)$ at night, and by Frederic Ducarme in December 2020 in the S passe at around $10 \mathrm{~m}$ depth $\left(12^{\circ} 52^{\prime} 17.4^{\prime \prime} \mathrm{S}-45^{\circ}\right.$ $\left.16^{\prime} 24.4^{\prime \prime} \mathrm{E}\right)$ at night.

Description. Rounded snout and jaw. Eye above the middle of the jaw. Grey iris. Anterior nostrils large and tubular, slightly protruding. Posterior nostril large and less protruding above anterior margin of the eye. Body almost cylindrical without dorsal fins. Color variable from blue gray speckled to light brown speckled. Estimated size between 100 to $130 \mathrm{~cm}$ TL.

Distribution and ecology. Seems to occur on several localities like Dar es Salaam (Tanzania) and Mombasa (Kenya) (2019 pers. comm. A Sutton); 


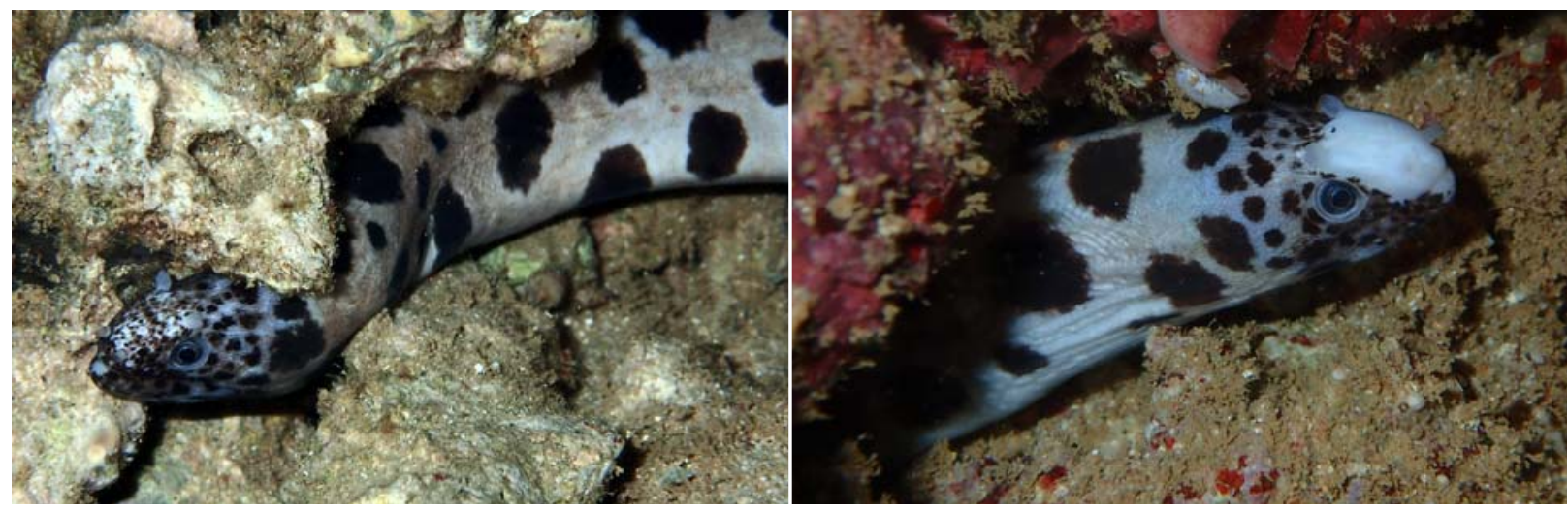

Figure 39. Uropterygius polyspilus different head pattern, Tsingoni beach reef $\left(12^{\circ} 47^{\prime} 16.2^{\prime \prime}\right.$ S-45 $05^{\prime} 31.7^{\prime \prime} \mathrm{E}$; 30 th June 2016 ; 20 h 38 min; 5 m depth).
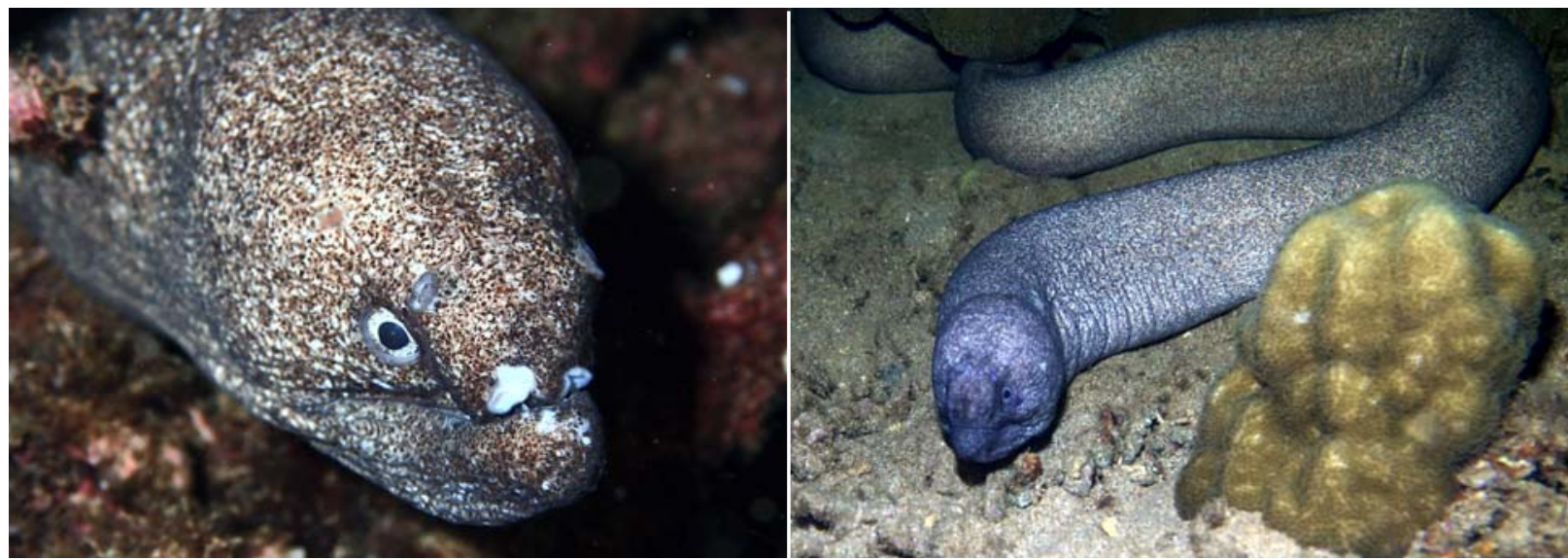

Figure 40. Left: Uropterygius sp. deformed head, at Chanfi beach reef (12 $45^{\prime} 19.3^{\prime \prime} \mathrm{S}-45^{\circ} 03^{\prime} 32.8^{\prime \prime} \mathrm{E} ; 27$ th February 2018 ; $21 \mathrm{~h}$ $14 \mathrm{~min} ; 2.5 \mathrm{~m}$ depth). Right: Uropterygius sp. on Sakouli beach reef flat (12 ${ }^{\circ} 53^{\prime} 15.9^{\prime \prime} \mathrm{S}-45^{\circ} 13^{\prime} 00.2^{\prime \prime} \mathrm{E} ; 27$ th January 2020; 22 h 33 min; depth: 2 m). Reproduced with permission from Nicolas Bouteiller.

Maldives (at $5 \mathrm{~m}$ depth, 2018 pers. comm. S Weinberg); Mauritius (visible in a video posted by the ProDive Mauritius Dive Center in 2015). Secretive. Freeze when approched. Seem to live in crevices of coral reefs or on reef flats between 2 to $20 \mathrm{~m}$ depth.

'Appears to have short rounded teeth although these eels never open their mouths very wide so it is difficult to tell. We can only presume that this moray feeds on either crustaceans or mollusks but this could be completely wrong' (2019 pers. comm. A Sutton).

\section{Family Ophichthidae}

Brachysomophis crocodilinus (Bennett, 1833)

Crocodile snake eel (EN);

Anguille-serpent crocodile (FR) (Figure 41)

Status at Mayotte. No record prior to this study.

Observation by divers. Observed very rarely. One time by Norbert Verneau in September 2006 on Papani beach coral sand $\left(12^{\circ} 46^{\prime} 25.3^{\prime \prime} \mathrm{S}-45^{\circ} 17^{\prime}\right.$ 41.9" E) at daytime. 


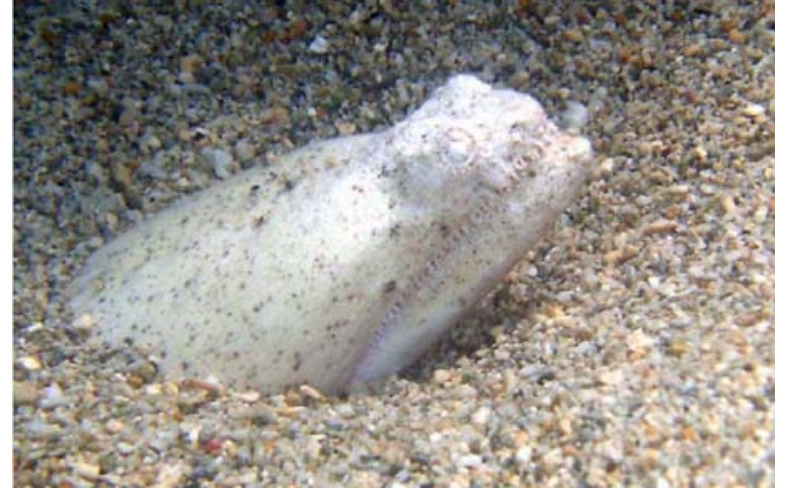

Figure 41. Brachysomophis crocodilinus on Papani beach

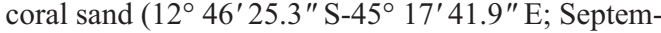
ber 2006; 13 h; 2 m depth). Reproduced with permission from Norbert Verneau.

Description. Eyes small, same color as rest of the head, and placed towards the front of the mouth. Protuberances on the lips (Lieske and Myers 2005). Snout very short but jaws elongated. Anterior nostril in a very short tube in upper lip. Lateral-line pores and temporal pores typically in dark spots; irregularly arranged smaller dark spots on flank between lateral line and dorsal fin of larger specimens (McCosker and Randall 2001). Maximum size $120 \mathrm{~cm}$ TL (Lieske and Myers 2005).

Distribution and ecology. East Africa, Madagascar and Mascarenes, east to Johnston Atoll and Society islands, Japan and Australia (Fricke et al. 2018). Generally found in shallow lagoon sand, rock and broken coral substrates at depths of 0-2 $\mathrm{m}$, but distributed from 0 to $30 \mathrm{~m}$ depth (McCosker and Randall 2001). Remain buried in sand with only the eyes protruding where it waits to ambush fish (Lieske and Myers 2005).

\section{Brachysomophis henshawi}

Jordan and Snyder, 1904

Reptilian Snake Eel (EN);

Anguille-serpent de Henshaw (FR) (Figure 42)

Status at Mayotte. First record from Mayotte by Allaria (2016).
Observationrs. Observed very rarely. Once by Daniel Budet before 2016 on Passe en S sand dune $\left(12^{\circ} 52^{\prime} 01.5^{\prime \prime} \mathrm{S}-45^{\circ} 16^{\prime} 20.2^{\prime \prime} \mathrm{E}\right)$ at daytime.

Description. Head flattened except for an abrupt depression behind the eye. Color variable from mottled red to yellowish or whitish (Allen et al. 2003). Snout short and jaws elongate. Nostrils in short tubes in upper lip and closely associated. Flesh above and behind eye laterally elevated as a ridge. Head pores and lateral-line pores apparent (McCosker and Randall 2001). Maximum size $120 \mathrm{~cm}$ TL (Bacchet et al. 2006).

Distribution and ecology. Indo-Pacific. Arabian sea to Hawaii, Marquesas and Society islands, French Polynesia, Japan to Coral Sea (Allen et al. 2003). Found over sandy areas, usually near or within coral or rocky reefs at depths from 1 to 35 m (McCosker and Randall 2001). Remain buried in sand with only the head protruding to ambush prey (Allen et al. 2003). Feed on fishes and crustaceans (Bacchet et al. 2006).

Callechelys marmorata (Bleeker, 1854)

Marbled snake eel (EN);

Anguille-serpent marbrée (FR) (Figure 43)

Status at Mayotte. First record from Mayotte by Allaria (2016).

Observation by the author. Observed rarely. E.g.: March 2016 Badamiers beach coral sand $\left(12^{\circ} 45^{\prime}\right.$ 33.8" S-45 ${ }^{\circ} 17^{\prime} 28.7^{\prime \prime}$ E) at daytime; May 2017 Passe en S sand $\left(12^{\circ} 52^{\prime} 01.5^{\prime \prime} \mathrm{S}-45^{\circ} 16^{\prime} 20.2^{\prime \prime} \mathrm{E}\right)$ at daytime; January 2018 Passe des îles Choizil on coral sand $\left(12^{\circ} 43^{\prime} 05.2^{\prime \prime} \mathrm{S}-44^{\circ} 59^{\prime} 30.4^{\prime \prime} \mathrm{E}\right)$ at daytime.

Description. Yellowish with numerous black spots and blotches. Short head, overhanging snout with prominent tubular nostrils. Elongated pupils. No pectoral fins. Maximum size $90 \mathrm{~cm}$ TL (Allen et al. 2003). 
Distribution and ecology. Red Sea, East Africa, Aldabra, Madagascar and western Mascarenes, east to Society and Marquesas islands, north to Taiwan, south to New Caledonia (Fricke et al. 2018). Found in shallow areas, beneath loose gravel and sand (Lieske and Myers 2005) at depths from 0 to $37 \mathrm{~m}$ (McCosker 1998). Also seen in lagoons and seaward sand patches, solitary, buried in sand with only the head protruding (Allen and Erdmann 2012).

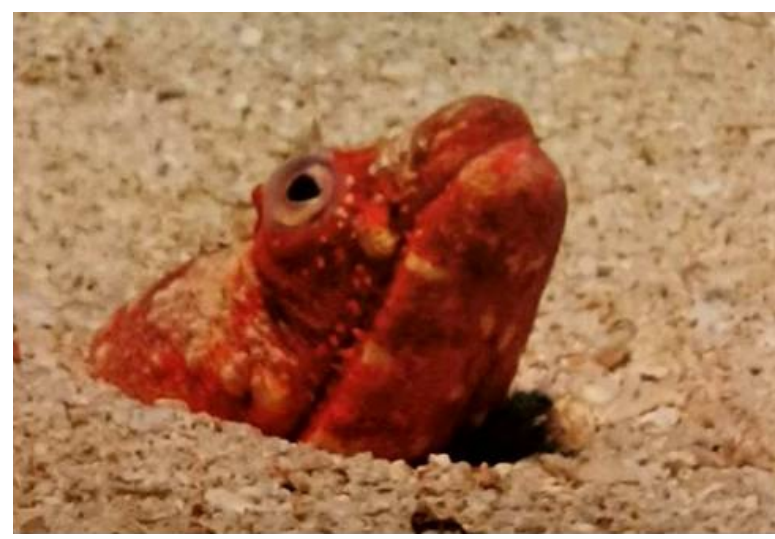

Figure 42. Brachysomophis henshawi on Passe en S sand dune (12 $52^{\prime} 01.5^{\prime \prime} \mathrm{S}-45^{\circ} 16^{\prime} 20.2^{\prime \prime} \mathrm{E}$, at daytime). Reproduced with permission from Daniel Budet.
Myrichthys colubrinus (Boddaert, 1781)

Harlequin snake eel (EN);

Anguille-serpent annelée (FR) (Figure 44)

Status at Mayotte. First record from Mayotte by Wickel and Jamon (2010). Subsequently reported from Mayotte by Wickel et al. (2014) and by Allaria (2016).

Observation by the author. Observed rarely. E.g.: October 2014 Bandrélé reef on reef flat $\left(12^{\circ} 54^{\prime}\right.$ $\left.33.1^{\prime \prime} \mathrm{S}-45^{\circ} 14^{\prime} 19.3^{\prime \prime} \mathrm{E}\right)$ at daytime; April 2017 Sada islet reef flat $\left(12^{\circ} 50^{\prime} 51.0^{\prime \prime} \mathrm{S}-45^{\circ} 05^{\prime} 18.2^{\prime \prime}\right.$ E) at night.

Description. White with brown to black bars (with black spots between bars in young individuals). Overhanging snout with tubular nostrils. Maximum size $90 \mathrm{~cm}$ TL (Allen et al. 2003). Resembles the venomous sea snake Laticauda colubrina, but is entirely harmless (Lieske and Myers 2005).

Distribution and ecology. Red Sea, East Africa, Seychelles, Comoros, Madagascar and Mascarenes, east to Johnston Atoll and Society is-
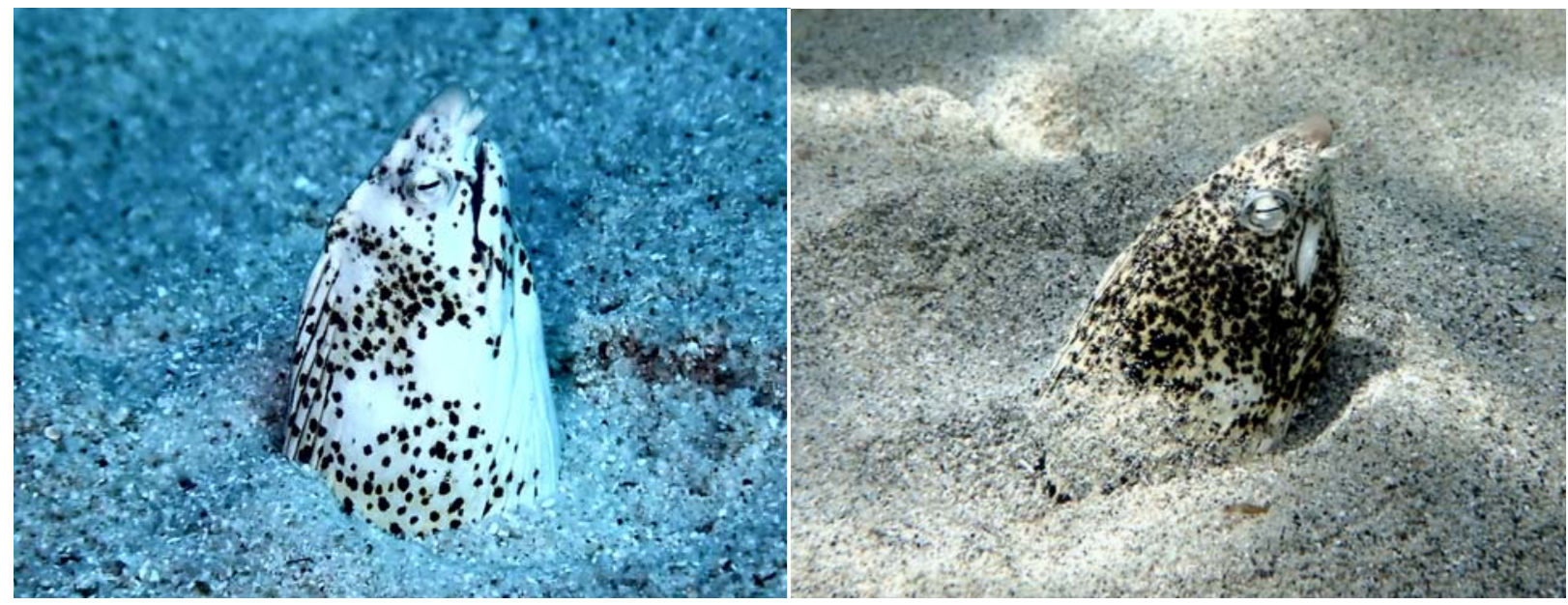

Figure 43. Left: Callechelys marmorata on Badamiers beach coral sand of tide pool $\left(12^{\circ} 45^{\prime} 33.8^{\prime \prime}\right.$ S- $45^{\circ} 17^{\prime} 28.7^{\prime \prime} \mathrm{E}$; 12 th March 2016; 13 h 55 min; depth: $1 \mathrm{~m})$. Right: C. marmorata at Passe des îles Choizil on coral sand (12 $43^{\prime} 05.2^{\prime \prime} \mathrm{S}-44^{\circ} 59^{\prime}$ 30.4 " E; 19th January 2018; 15 h; 16 m depth). 

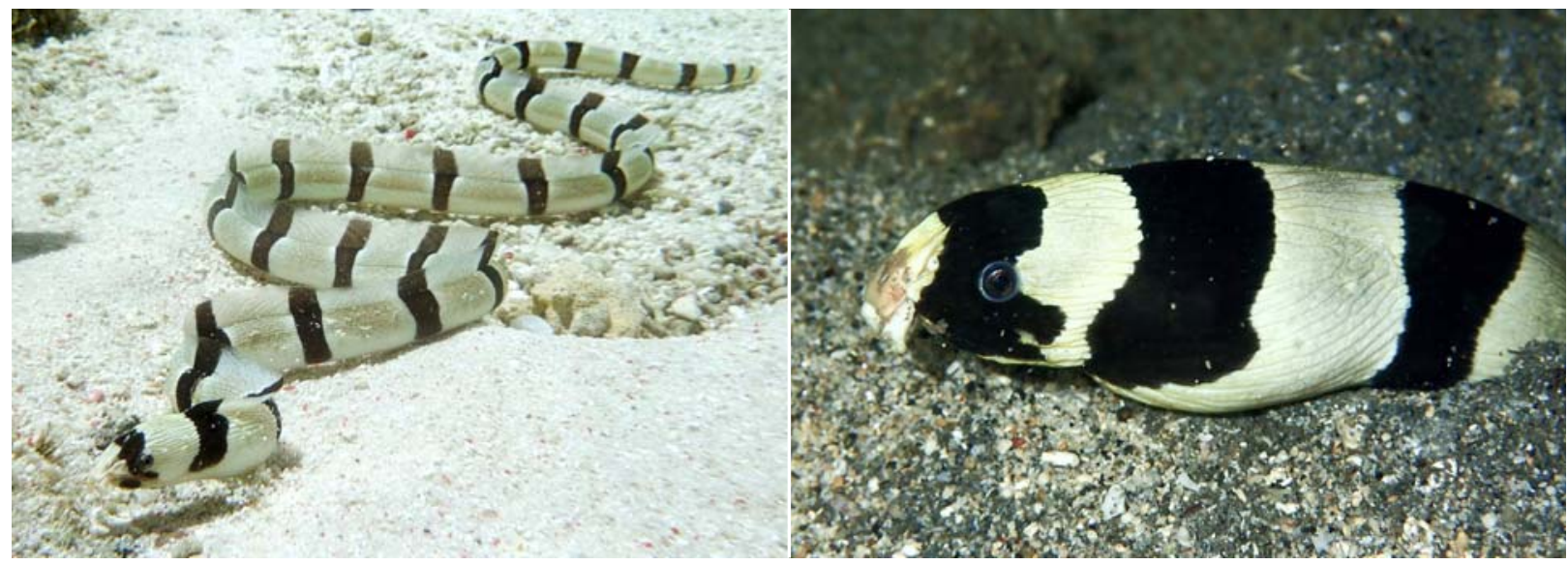

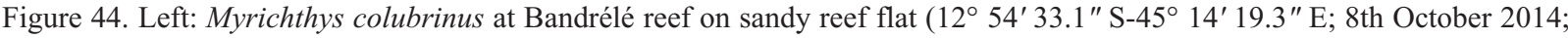

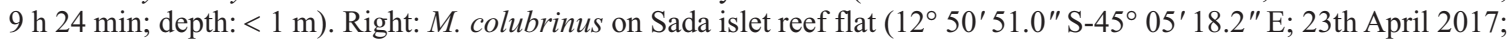
19 h 42 min; < 1 m depth).

lands, north to Ryukyu and Ogasawara islands, Australia (Fricke et al. 2018). Found in shallow sandy flats and seagrass beds (Lieske and Myers 2005). Occurs from 0 to $35 \mathrm{~m}$ depth. Feed on small fishes and crustaceans (Bacchet et al. 2006).

\section{Myrichthys maculosus (Cuvier, 1816)}

Spotted Snake Eel (EN);

Anguille-serpent maculée (FR) (Figure 45)

Status at Mayotte. First record from Mayotte by Wickel et al. (2014).

Observation by the author. Observed commonly. E.g.: March 2015 on Sakouli reef flat $\left(12^{\circ} 53^{\prime}\right.$ $\left.19.6^{\prime \prime} \mathrm{S}-45^{\circ} 12^{\prime} 54.2^{\prime \prime} \mathrm{E}\right)$ at night; February 2016 White sand islet reef $\left(12^{\circ} 59^{\prime} 10.5^{\prime \prime} \mathrm{S}-45^{\circ} 12^{\prime}\right.$ 52.7" E) at daytime; November 2016 Passe en $\mathrm{S}$ reef $\left(12^{\circ} 52^{\prime} 09.1^{\prime \prime} \mathrm{S}-45^{\circ} 16^{\prime} 30.1^{\prime \prime} \mathrm{E}\right)$ at night; May 2017 Bandrélé islet reef flat (12० 53' 55.0" S-45 $13^{\prime} 44.8^{\prime \prime}$ E) at daytime; November 2017 Sakouli beach reef flat $\left(12^{\circ} 53^{\prime} 17.5^{\prime \prime} \mathrm{S}-45^{\circ} 12^{\prime}\right.$ $53.3 " \mathrm{E}$ ) at night.

Description. Yellowish to cream with large dark oval spots. Overhanging snout with long tubular nostrils. Small pectoral fins. Maximum siza 100 cm TL (Allen et al. 2003). Smaller and more numerous spots with age (Lieske and Myers 2005).

Distribution and ecology. Red Sea, East and South Africa, Aldabra, Madagascar and Mascarenes, east to Panama (except for Hawaiian Islands), Japan, Ogasawara Islands and Midway Atoll, Australia, Lord Howe and Kermadec Islands (Fricke et al. 2018). Found in sandy areas of reef flats, lagoons, and seaward reefs at depths from 0 to $262 \mathrm{~m}$. Lives buried in the sand, more commonly observed at night (Lieske and Myers 2005). Feed on small fishes and crustaceans (Bacchet et al. 2006).

Ophichthus altipennis (Kaup, 1856)

Highfin snake eel (EN) (Figure 46)

Status at Mayotte. Never recorded prior to this study.

Observation by the author. Observed very rarely. Two times: April 20183 Baobabs beach sand (12 $\left.56^{\prime} 48.2^{\prime \prime} \mathrm{S}-45^{\circ} 05^{\prime} 58.1^{\prime \prime} \mathrm{E}\right)$ at night; June $2019 \mathrm{~N}^{\prime}$ gouja beach sand $\left(12^{\circ} 57^{\prime} 47.1^{\prime \prime} \mathrm{S}-45^{\circ} 05^{\prime}\right.$ $03.1^{\prime \prime}$ E) at night. 


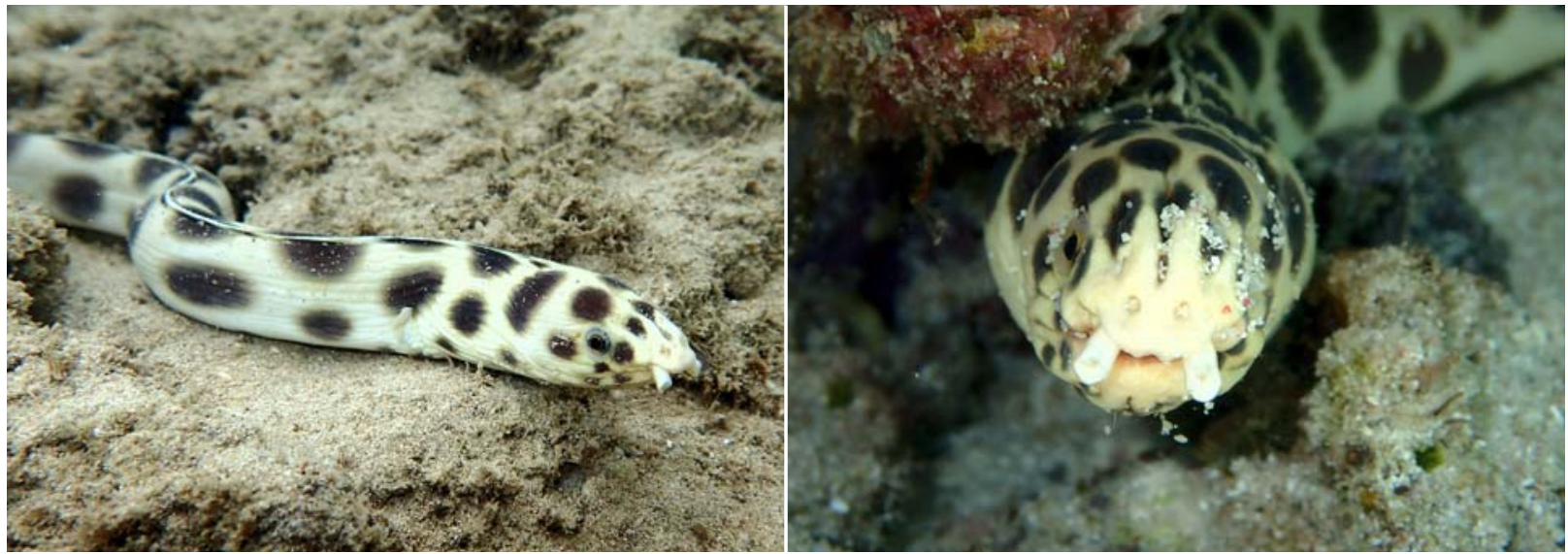

Figure 45. Left: Myrichthys maculosus on Bandrélé islet reef flat (12 ${ }^{\circ} 53^{\prime} 55.0^{\prime \prime}$ S-45 $13^{\prime} 44.8^{\prime \prime}$ E; 26th May $2017 ; 11$ h 17 min; depth: $2 \mathrm{~m})$. Right: M. maculosus at White sand islet reef $\left(12^{\circ} 59^{\prime} 10.5^{\prime \prime} \mathrm{S}-45^{\circ} 12^{\prime} 52.7^{\prime \prime} \mathrm{E}\right.$; 28th February $2016 ; 13 \mathrm{~h}$ $34 \mathrm{~min} ; 1.5 \mathrm{~m}$ depth).
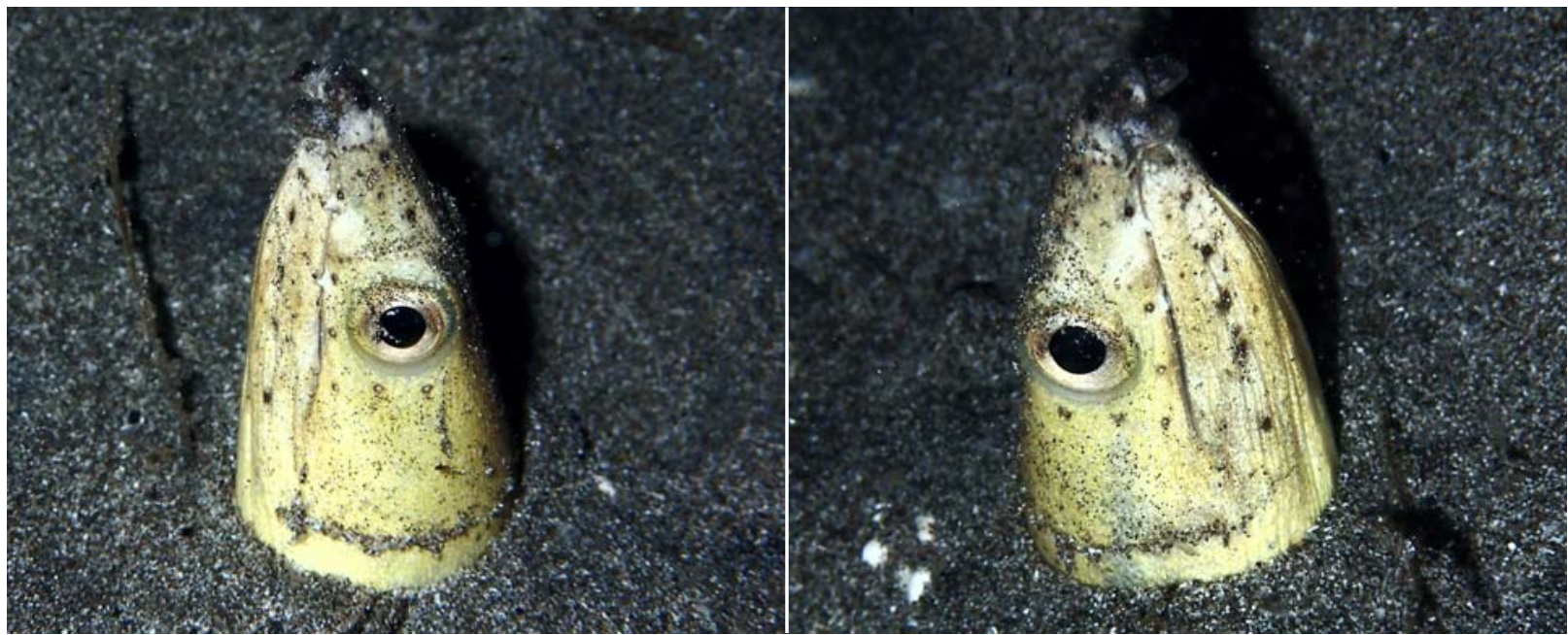

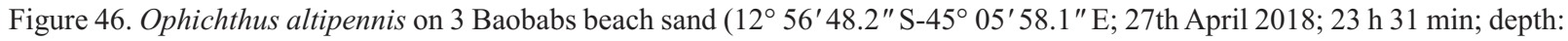
$1 \mathrm{~m})$. Left: left side of the head; right: right side of the head.

Description. Color tan to brown. Pectoral fins dark but those of smaller specimens may be clear. A prominent pale spot before eye and a smaller pale spot on snout above anterior nostril. Barbels prominent along ventral margin of upper lip, one beneath the anterior margin of the eye, the other midway between it and the base of the anterior nostril. Maximum size $120 \mathrm{~cm}$ TL (Bacchet et al. 2006).
Distribution and ecology. Indo-Pacific: Australia, French Polynesia, Indonesia, Japan, the Marshall Islands, Malaysia, the Philippines, and Papua New Guinea (McCosker and Randall 2002). Benthic and burrowing species which occurs over soft bottoms of inshore waters (Hoese et al. 2006) at depths between 1 to $40 \mathrm{~m}$ (McCosker and Randall 2002). Often buried in sand with only the head protruding (Allen and Erdmann 2012). 
Feeds on cephalopodes, crustaceans, and small fishes (Bacchet et al. 2006).

Ophichthus bonaparti (Kaup, 1856)

Napoleon Snake Eel (EN);

Anguille-serpent de Napoléon (FR) (Figure 47)

Status at Mayotte. First record from Mayotte by Fouquet (2000). Subsequently reported from Mayotte by Wickel and Jamon (2010) and Wickel et al. (2014).

Observations. Observed very rarely. Two observations by Norbert Verneau on Musicale beach $\left(12^{\circ} 55^{\prime} 09.7^{\prime \prime} \mathrm{S}-45^{\circ} 11^{\prime} 09.7^{\prime \prime} \mathrm{E}\right)$ by the end of the day and in Badamiers sand lagoon (3 $\mathrm{m}$ depth).

Description. Dirty white with dark brown encircling bars and occasional spots between. Head has dark-edge bronze spots and blotches. Maximum size $75 \mathrm{~cm}$ TL (Allen et al. 2003).

Distribution and ecology. Indo-Pacific: East and South Africa, Mauritius, Indonesia, Society Islands, Japan, south to Australia (Allen et al. 2003). Found in sand flats of lagoon and seaward reefs to depths greater than $20 \mathrm{~m}$ (Myers 1999). Often buried in sand with only the head protrud-

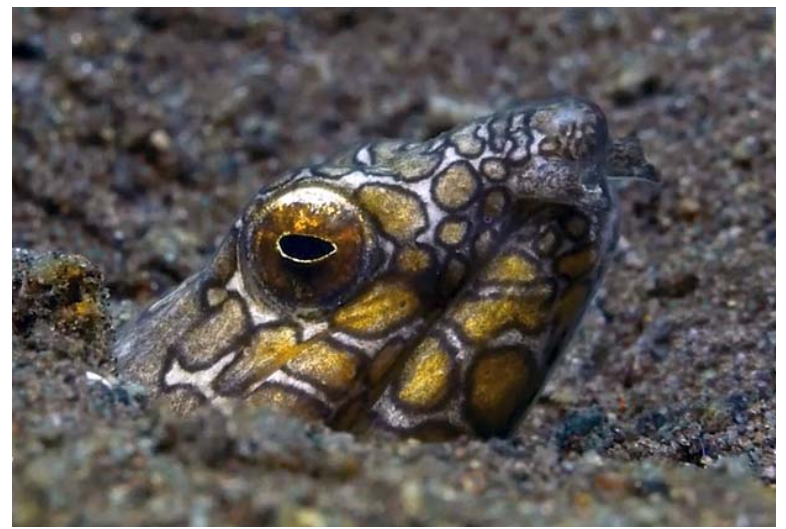

Figure 47. Ophichthus bonaparti at Musicale beach $\left(12^{\circ} 55^{\prime}\right.$ 07.8" S-45 $11^{\prime}$ 07.9" E; 3 m depth). Reproduced with permission from Norbert Verneau. ing. Feed on small fishes, and probably crustaceans (Bacchet et al. 2006).

Pisodonophis cancrivorus (Richardson, 1848) Longfin snake eel (EN);

Serpenton à longue nageoire (FR) (Figure 48)

Status at Mayotte. Never recorded prior to this study.

Observation by the author. Observed very rarely. One time in November 2018 at Airport seaward reef $\left(12^{\circ} 49^{\prime} 08.3^{\prime \prime} \mathrm{S}-45^{\circ} 17^{\prime} 32.3^{\prime \prime} \mathrm{E}\right)$ at night.

Description. Gray to brown, covered with skin wrinkles highlighted by pale and darker shades. Overhanging snout with tubular nostriles. Cylindrical body, compressed only along extreme tail tip. Maximum size $75 \mathrm{~cm}$ TL (Allen et al. 2003).

Distribution and ecology. Indo-pacific: Red Sea, East and South Africa, Madagascar and western Mascarenes, east to French Polynesia, Japan, Australia (Fricke et al. 2018). Occur in lagoons and estuaries, entering freshwater. Found in coastal sand and silt patches in 1 to $25 \mathrm{~m}$ depth. Often buried in sand with only the head protruding (Allen et al. 2003).

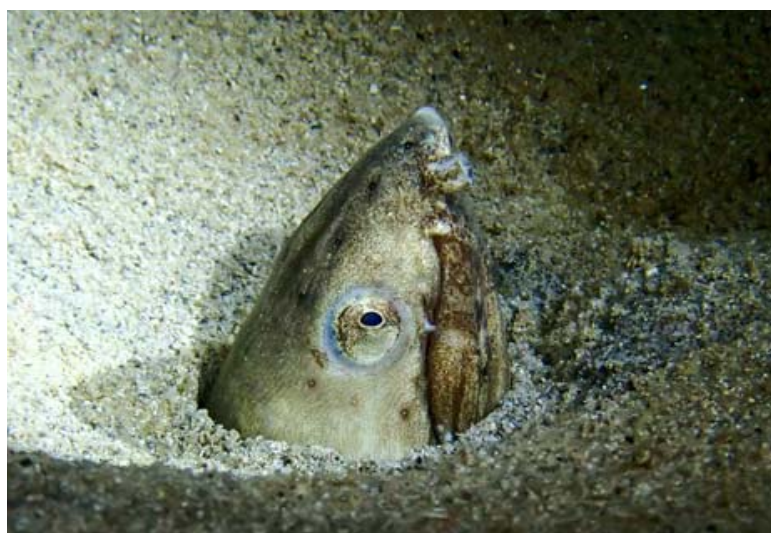

Figure 48. Pisodonophis cancrivorus at Airport seaward reef on sandy bottom $\left(12^{\circ} 49^{\prime} 08.3^{\prime \prime} \mathrm{S}-45^{\circ} 17^{\prime} 32.3^{\prime \prime} \mathrm{E}\right.$; 30th November 2018; 18 h $38 \mathrm{~min} ; 12 \mathrm{~m}$ depth). 


\section{Family Synaphobranchidae}

Meadia abyssalis (Kamohara, 1938)

Abyssal cutthroat eel (EN); anguille fardée abyssale (FR) (Figure 49)

Status at Mayotte. First record from Mayotte by Wickel and Jamon (2010) from fisherman observations.

Observation by the author. No observation. Observed very rarely, by fishermen.

Description. Body color gray to black, pale below. Upper jaw longer than lower jaw. Rictus posterior to eye. Anterior nostril tubular, posterior nostril a hole anterior from the eye. Pectoral fin well developed. Dorsal fin originating posterior from the anus (Lai 2017). Maximum size $73 \mathrm{~cm}$ TL (Masuda et al. 1984).

Distribution and ecology. Indo-Pacific: Reunion, Mauritius, southern Japan, Hancock Seamount, and the Hawaiian and Society islands. Off Southern Brazil (Bernardes et al. 2005). Occurs on the continental slope. Benthopelagic species at depth ranging from 100 to $329 \mathrm{~m}$ (Mundy 2005).

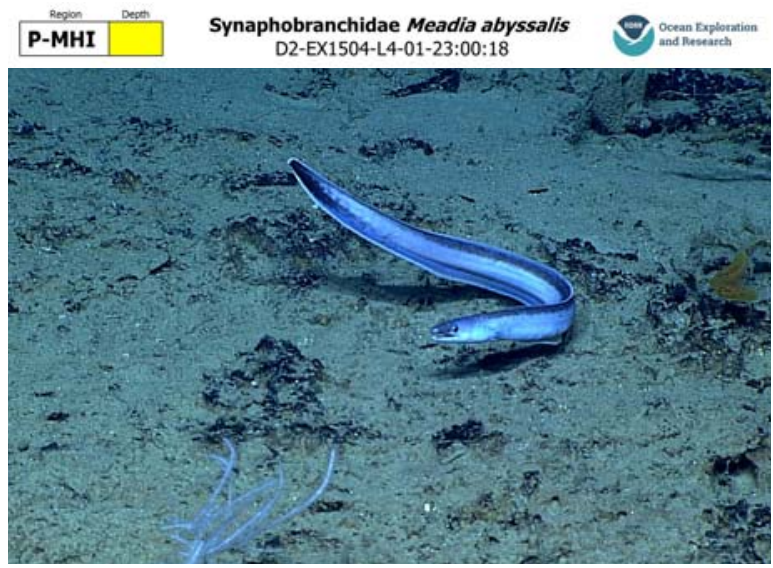

Figure 49. Meadia abyssalis. Taken from https://www.ncei.noaa. gov/waf/okeanos-animal-guide/Synaphobranchidae 011.html.

\section{DISCUSSION}

This illustrated checklist provides an up-todate census of Anguilliformes of Mayotte lagoon. A total of 47 species were recorded combining literature data, my own underwater visual censuses, and records from other divers. Fourteen new species for Mayotte were recorded. Although the presence of some of these species had already been suspected, no observations had been made. These new observations, therefore, improved our knowledge of this understudied group and provide new locality data for some species. Some observations are of particular interest, such as Uropterygius nagoensis, which was only known from the Western Pacific Ocean (Smith et al. 2019), and a new species of Uropterygius yet to describe which seems to occur in a large area of the West Indian Ocean. More intensive survey and further identification by morphological characteristics and DNA analysis will be useful to clarify this point.

In Mayotte, Anguilliformes are dominated by the Family Muraenidae, which globally represent only around 25\% of all Anguilliformes (ca. 202 Muraenidae out of 820 Anguilliforms). This could be an observational bias due to the behavior of this group, which is more diurnal and inclined to come out of its hiding place compared to other families like Ophichtidae, the second most common representative of Anguilliformes that lives hidden most of the day. The low rate of observation of two freshwater species of Anguillidae could be due to the rarity of these species but also to the lack of research in inland streams. Thus, there is a need to focus also on these habitats of Mayotte to reveal the true extent of its distribution.

The proportion of Muraenidae among all fish species of Mayotte $(3.82 \%)$ is close to that of the other islands of the region like Réunion and Mauritius. Even if the proportion of Muraenidae found in Mayotte is close to the proportions found in 
other islands in the Western Indian Ocean, the number of reef fish species in Mayotte still seems to be underestimated. This is especially true as some pictures have not been kept because of their lack of clarity to identify the species. I personally identified several species new to the Mayotte lagoon in other taxa, and most of these observations were implemented in the TSIONO database (https://www.tsiono.fr), a participative observation network managed by the Parc Naturel Marin de Mayotte. Therefore, a lot of new discoveries remain to be made on this forgotten part of the world and research project should be developed to increase the survey effort on different marine groups. Using citizen science, such as taking pictures of encountered species, could really help identify the species community in a region. Nevertheless, even if photography may be a good method to avoid sampling, it may not be sufficient for species with cryptic morphological criteria, but it could highlight this part of the globe for future research. Thus, these observations need to be taken with care and should be confirmed with sampled specimens or tissues. Gathering knowledge could also help to understand the impact of recent volcanic activity close to Mayotte (Cesca et al. 2020) and the global warming on fish populations (Overland et al. 2010; Alfonso et al. 2021).

\section{ACKNOWLEDGEMENTS}

First, I thank Norbert Verneau for providing essential data, advice, and good pictures. I thank Frederic Ducarme, Yannick Stefan and Nicolas Bouteiller for providing observations and for sharing time with me underwater. I am also thankful to Daniel Budet and Pierre Valade for sharing their photos and data. A special thanks to Stephane Lapos, Baptiste Vernier, Daniel Hauck, Paul Deparis and many other for spending hours with me underwater. Thanks to Oliver Hawlitschek for the proofreading and comments. And finally, I would like to thank my wife for letting me spend more than $352 \mathrm{~h}$ underwater and not be worried when I spent three consecutive hours in water at night. I also thank Alan Sutton is an underwater photographer and writer at Seaunseen (https://seaunseen.com/granite-moray-eel/), and Steve Weinberg holds a PhD at the University of Amsterdam (1979), writer and photographer, specialist in the underwater world and travel, author of over 30 books and about 600 mazine articles (http://www.weinberg.lu/biographie-de-stevenweinberg). Finally, I thank all the diving clubs that brought me to the amazing dive sites, and the first Hippocampe plongée, Lagon Maore, Happy Divers, Jolly Roger, Abalone plongée and Nyamba club.

\section{REFERENCES}

Alfonso S, Gesto M, Sadoul B. 2021. Temperature increase and its effects on fish stress physiology in the context of global warming. J Fish Biol. 98: 1496-1508.

Allaria M. 2016. Poissons de Mayotte. Guide Océan Indien. 388 p.

Allen GR, ERdMANN MV. 2012. Reef fishes of the East Indies. Volumes I-III. Perth, Australia: Universitiy of Hawai'i Press, Tropical Reef Research.

Allen G, Steene R, Humann P, Deloach N. 2003. Reef fish identification. Tropical Pacific. Florida: New World Publications. 480 p. Amirault G, Lamalfa-Diaz M, Soumille O. 2020. ZNIEFF inventories (Zone Naturelle d'Intérêt Ecologique, Faunistique et Floristique) INPN (Inventaire National du Patrimoine Naturel). https://inpn.mnhn.fr/zone/ znieff/060000026.

BACCHET P, ZysMAN T, LefÈVRE Y. 2006. Guide des poissons de Tahiti et ses îles. Tahiti (Polynésie Francaise): Éditions $\mathrm{Au}$ Vent des Îles. $608 \mathrm{p}$. 
Bernardes RÁ, De Figueiredo JL, Rodrigues AR, Fischer LG, VoORen CM, Haimovici M, Rossi-Wongtschowski CLDB. 2005. Peixes de zona econômica exclusiva da região sudeste-sul do Brasil: levantamento com armadilhas, pargueiras e rede de arrasto de fundo. São Paulo: Editora da Universidade de São Paulo. 295 p.

BöHLKe EB, Randall JE. 2000. A review of the Moray eels (Angulliformes: Muraenidae) of the Hawaiian Islands, with descriptions of two new species. Proceedings of the Academy of Natural Sciences of Philadelphia. Vol. 150. p. 203-278.

BRAY DJ. 2020a. Coloconger scholesi in Fishes of Australia. [accessed 2020 April 27]. https:// fishesofaustralia.net.au/home/species/3452.

BRAY DJ. 2020b. Muraenesox bagio in Fishes of Australia. [accessed 29 April 2020]. https:// fishesofaustralia.net.au/home/species/2042.

Castle PHJ. 1986. Congridae. In: Smith MM, HEEMSTRA PC, editors. Smiths' sea fishes. Berlin: Springer-Verlag. p. 161-165.

Cesca S, Letort J, Razafindrakoto HNT, Heimann S, Rivalta E, Isken MP, Dahm T. 2020. Drainage of a deepmagma reservoir near Mayotte inferred from seismicity anddeformation. Nat Geosci. 13 (1): 87-93. doi:10. 1038/s41561-019-0505-5

Chen H-M, Shao K-T, Chen CT. 1994. A review of the muraenid eels (Family Muraenidae) from Taiwan with descriptions of twelve new records. Zool Stud. 33 (1): 44-64.

Deliot G. 2000. Premier inventaire ichtyologique des zones de fonds de baies de l'île de Mayotte 'secteur à mangrove' (océan Indien-canal du Mozambique-Archipel des Comores). Service des Pêches et de l'Environnement Marin. Université du Littoral Côte d'Opale. 37 p.

DORIS. 2020. Données d'Observations pour la Reconnaissance et l'Identification de la faune et la flore Subaquatiques. [accessed 2020 September]. https://doris.ffessm.fr.

FAHMI M. 2015. Short communication conserva- tion genetic of tropical eel in Indonesian Waters based on population genetic study. Proceedings National Seminar of Biodiversity Community of Indonesia. p 38-43.

FouQueT P. 2000. Situation de la pêche artisanale à Mayotte en l'an 2000. Analyse statistique du système de suivi de l'activité halieutique (janvier 1997-juin 2000). Rapport SPEM/DAF. $67 \mathrm{p}$.

FOURMANOIR P. 1954. Ichtyologie et pêche aux Comores. Mémoires de l'Institut scientifique de Madagascar. Série A. Tome 9: 187-238.

Fricke R, Kulbicki M, WANTIEZ L. 2011. Checklist of the fishes of New Caledonia, and their distribution in the Southwest Pacific Ocean (Pisces). Stuttgarter Beiträge zur Naturkunde A, Neue Serie. 4: 341-463.

Fricke R, Mahafina J, Behivoke F, JaOnalison H, LÉOPOlD M, Ponton D. 2018. Annotated checklist of the fishes of Madagascar, southwestern Indian Ocean, with 158 new records. FishTaxa. 3 (1): 1-432.

Froese R, Pauly D. 2020. FishBase. Anguilliformes. World Register of Marine Species. [accessed 2020 April 20]. https://www.marine species.org/aphia.php?p=taxdetails\&id=10295.

Hoese DF, Bray DJ, Paxton JR, Allen GR. 2006. Fishes. In: BeAsley OL, Wells A, editors. Zoological catalogue of Australia. Vol. 35. Australia. ABRS \& CSIRO Publishing. $2178 \mathrm{p}$.

Horton T, Kroh A, Ahyong S, Bailly N, Bieler $\mathrm{R}$, Boyko CB, Brandão SN, Gofas S, HoOPER JNA, HERNANDEZ F, et al. 2020. World Register of Marine Species. [accessed 2020 September 10]. http://www.marinespecies.org. doi: $10.14284 / 170$

[INSEE] Institut National de la Statistique ET DEUS ÉTUDES ÉCONOMIQUES. 2021. [accessed 2021 March]. https://www.insee.fr/fr/statistiques.

KARRER C. 1982. Anguilliformes du Canal de Mozambique (Pisces, Teleostei). Faune Tropicale 23: 1-116. 
Keith P, Marquet G, Valade P, Bosc P, Vigneux E. 2006. Atlas des poissons et des crustacés d'eau douce des Comores, Mascareignes et Seychelles. Publications scientifiques du MNHN, Patrimoine Naturels. 65. 250 p.

LAI K. 2017. The National Checklist of Taiwan. Taiwan biodiversity information facility (TaiBIF). Checklist dataset. [accessed 2020 May 13]. doi:10.15468/auw1kd

Letourneur Y, MagGiorani J-M. 1995. Effets de la mise en réserve de la passe en $\mathrm{S}$ (Ile de Mayotte) sur les peuplements et populations de poissons. Rapport Neptune Services, La Réunion \& Service des Pêches, Mayotte. 24 p.

Lieske E, Myers RF. 2005. Guide des poissons des récifs coralliens. Les guides du naturaliste. Delachaux et Niestlé. 398 p.

Masuda H, Amaoka K, Araga C, Uyeno T, YosHINO T. 1984. The fishes of the Japanese Archipelago. Vol. 1. Tokyo: Tokai University Press. 437 p.

McCosker JE. 1998. A revision of the snake-eel genus Callechelys (Anguilliformes: Ophichthidae) with the description of two new IndoPacific species and a new Callechelyin genus. Proc Calif Acad Sci. 50 (7): 185-215.

McCosker JE, Randall JE. 2001. Revision of the snake-eel genus Brachysomophis (Anguilliformes: Ophichthidae), with description of two new species and comments on the species of Mystriophis. Indo-Pac Fish. (33): 1-32.

McCosker JE, Randall JE. 2002. Ophichthys melanochir Bleeker, 1865, a junior synonym of the highfin snake eel Ophichthus altipennis (Kaup, 1856). Copeia. 3: 798-799.

MichON L. 2016. The volcanism of the Comores archipelago integrated at a regional scale. In: BACHELERY P, LÉNAT J-F, Di MuRO A, Michon $\mathrm{L}$, editors. Active volcanoes of the Southwest Indian Ocean: Piton de la Fournaise and Karthala. Active volcanoes of the World. Springer-Verlag. p. 233-244.

MNHN-OFB, ed. 2020. National inventory of natural heritage (INPN). Muséum National
d'Histoire Naturelle. [accessed 2020 October 14]. https://inpn.mnhn.fr.

Mundy BC. 2005. Checklist of the fishes of the Hawaiian Archipelago. Bishop Mus Bull Zool (6): 1-704.

Myers RF. 1991. Micronesian reef fishes. 2nd ed. Barrigada: Coral Graphics. 298 p.

MYers RF. 1999. Micronesian reef fishes: a comprehensive guide to the coral reef fishes of Micronesia. 3rd ed. Barrigada: Coral Graphics. 330 p.

Overland Je, Alheit J, Bakun A, Hurrell JW, Mackas DL, Miller AJ. 2010. Climate controls on marine ecosystems and fish populations. J Mar Sys. 79: 305-315.

RANDALL JE. 2005. Reef and shore fishes of the South Pacific. New Caledonia to Tahiti and the Pitcairn Islands. Honolulu: University of Hawaii Press. 720 p.

Sмith DG. 1997. Muraenesocidae. Pike congers. In: CARPENTER KE, NiEM VH, editors. FAO species identification guide for fishery purposes. The living marine resources of the WCP. Vol. 3. Batoid fishes, chimaeras and bony fishes part 1 (Elopidae to Linophrynidae). Rome: FAO. p. 1673-1677.

Smith DG. 1999. Congridae. Conger eels. In: CARPENTER KE, NiEM VH, editors. FAO species identification guide for fishery purposes. The living marine resources of the WCP. Vol. 3. Batoid fishes, chimaeras and bony fishes part 1 (Elopidae to Linophrynidae). Rome: FAO. p. 1680-1686.

Smith DG, Bogorodsky SV, Mal AO, AlperMANN TJ. 2019. Review of the moray eels (Anguilliformes: Muraenidae) of the Red Sea, with description of a new species. Zootaxa. 4704 (1): 1-87.

TALWAR PK, JHINGRAN AG. 1991. Inland fishes of India and adjacent countries. Vol. 1. Rotterdam: A.A. Balkema. 541 p.

WiCKEL J. 2004. Inventaire des poissons, scléractiniaires et mammifères marins identifiés à Mayotte (Océan Indien). Rapport DAF/ 
SPEM. $21 \mathrm{p}$.

WiCKEL J, JAMON A. 2010. Inventaire taxonomique actualisé des poissons marins de l'île de Mayotte et des bancs récifaux de GeyserZélée, Canal de Mozambique. Liste révisée des espèces et élaboration d'une base de données fonctionnelle. Rapport LAGONIA/APNEEAquarium de la Réunion. 34 p.

Wickel J, Jamon A, Pinault M, Durville P,
Chabanet P. 2014. Composition et structure des peuplements ichtyologiques marins de l'île de Mayotte (sud-ouest de l'océan Indien). Cybium. 38 (3): 179-203.

Yamada U, Shirai S, Irie T, Tokimura M, Deng S, Zheng Y, Li C, KiM YU, Kim YS. 1995. Names and illustrations of fishes from the East China Sea and the Yellow Sea. Tokyo: Overseas Fishery Cooperation Foundation. 288 p. 
